\title{
FINITE ELEMENT APPROXIMATION OF A TWO-LAYERED LIQUID FILM IN THE PRESENCE OF INSOLUBLE SURFACTANTS *
}

\author{
John W. BARretT ${ }^{1}$ AND Linda El Alaoui ${ }^{1}$
}

\begin{abstract}
We consider a system of degenerate parabolic equations modelling a thin film, consisting of two layers of immiscible Newtonian liquids, on a solid horizontal substrate. In addition, the model includes the presence of insoluble surfactants on both the free liquid-liquid and liquid-air interfaces, and the presence of both attractive and repulsive van der Waals forces in terms of the heights of the two layers. We show that this system formally satisfies a Lyapunov structure, and a second energy inequality controlling the Laplacian of the liquid heights. We introduce a fully practical finite element approximation of this nonlinear degenerate parabolic system, that satisfies discrete analogues of these energy inequalities. Finally, we prove convergence of this approximation, and hence existence of a solution to this nonlinear degenerate parabolic system.
\end{abstract}

Mathematics Subject Classification. 65M60, 65M12, 35K55, 35K65, 35K35, 76A20, 76D08.

Received April 24, 2007.

Published online July 30, 2008.

\section{INTRODUCTION}

In $[1,2]$ fully practical finite element approximations were proposed and analysed for a system of nonlinear degenerate parabolic equations modelling a thin film of liquid, laden with insoluble surfactant, on a horizontal substrate in the possible presence of both attractive and repulsive van der Waals forces. In this paper, we extend the approximation and subsequent analysis in [1] to the case when the thin film consists of two layers of immiscible Newtonian liquids with possibly different viscosities. In addition, the model includes the presence of insoluble surfactants on both the free liquid-liquid and liquid-air interfaces, and the presence of both attractive and repulsive van der Waals forces in terms of the heights of the two layers, and possibly the total height of the film.

The model problem, derived using lubrication theory, as it appears in the applied mathematics, physics and engineering literature, see e.g. [4], is the following: Find $\left\{u_{i}(x, t), v_{i}(x, t), w_{i}(x, t)\right\}_{i=1}^{2}$ such that

$$
\mu \frac{\partial u_{1}}{\partial t}=\nabla \cdot\left[\frac{1}{3} u_{1}^{3} \nabla w_{1}+\frac{1}{2} u_{1}^{2} u_{2} \nabla w_{2}-\frac{1}{2} u_{1}^{2} \nabla\left(\sigma_{1}\left(v_{1}\right)+\sigma_{2}\left(v_{2}\right)\right)\right],
$$

\footnotetext{
Keywords and phrases. Thin film, surfactant, bilayer, fourth order degenerate parabolic system, finite elements, convergence analysis.

* Supported by EPSRC U.K. grant GR/S35660/01.

${ }^{1}$ Department of Mathematics, Imperial College, London, SW7 2AZ, UK. jwb@ic.ac.uk
} 


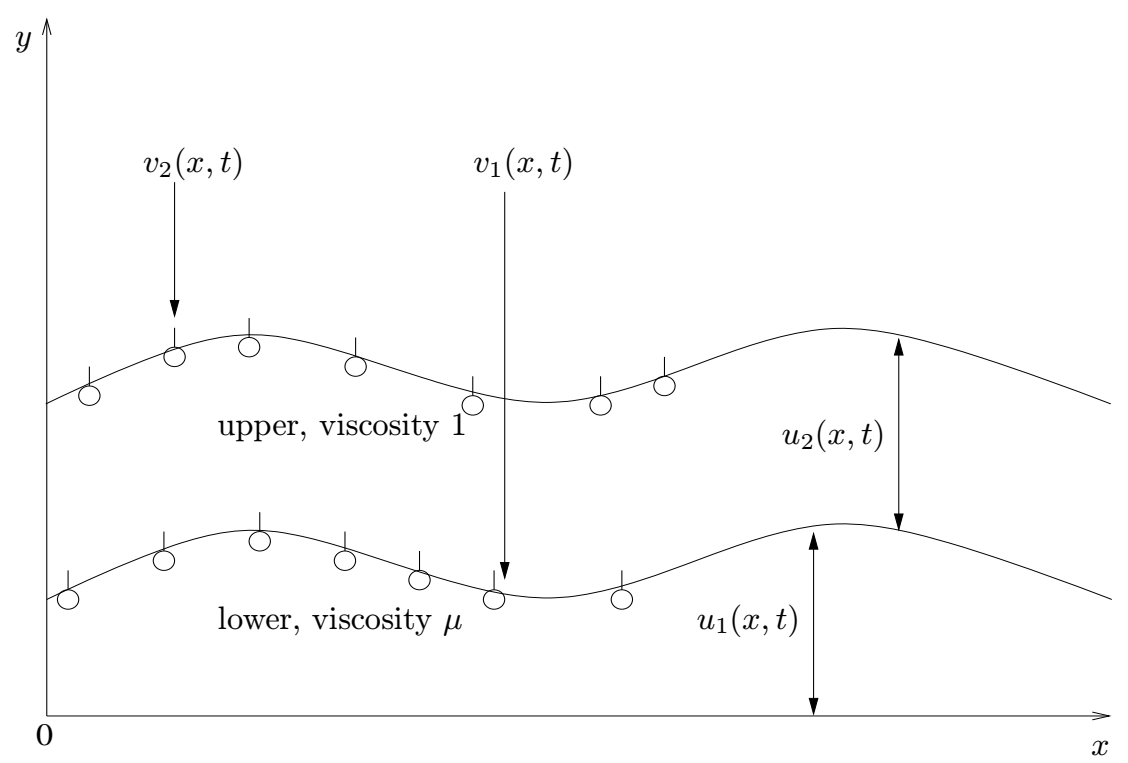

Figure 1. Geometry of the two-layered system.

$$
\begin{aligned}
\mu \frac{\partial u_{2}}{\partial t}= & \nabla \cdot\left[\frac{1}{2} u_{1}^{2} u_{2} \nabla w_{1}+u_{1} u_{2}^{2} \nabla w_{2}-u_{1} u_{2} \nabla\left(\sigma_{1}\left(v_{1}\right)+\sigma_{2}\left(v_{2}\right)\right)\right]+\mu \nabla \cdot\left[\frac{1}{3} u_{2}^{3} \nabla w_{2}-\frac{1}{2} u_{2}^{2} \nabla \sigma_{2}\left(v_{2}\right)\right], \\
w_{1}-w_{2}= & -c_{1} \Delta u_{1}+\phi_{1}\left(u_{1}\right)-\phi_{2}\left(u_{2}\right), \\
w_{2}= & -c_{2} \Delta\left(u_{1}+u_{2}\right)+\phi_{2}\left(u_{2}\right)+\phi_{3}\left(u_{1}+u_{2}\right), \\
\mu \frac{\partial v_{1}}{\partial t}= & \rho_{1} \mu \Delta v_{1}+\nabla \cdot\left[\frac{1}{2} u_{1}^{2} v_{1} \nabla w_{1}+u_{1} v_{1}\left(u_{2} \nabla w_{2}-\nabla\left[\sigma_{1}\left(v_{1}\right)+\sigma_{2}\left(v_{2}\right)\right]\right)\right] \\
\mu \frac{\partial v_{2}}{\partial t}= & \rho_{2} \mu \Delta v_{2}+\nabla \cdot\left[\frac{1}{2} u_{1}^{2} v_{2} \nabla w_{1}+u_{1} v_{2}\left(u_{2} \nabla w_{2}-\nabla\left[\sigma_{1}\left(v_{1}\right)+\sigma_{2}\left(v_{2}\right)\right]\right)\right] \\
& +\mu \nabla \cdot\left[\frac{1}{2} u_{2}^{2} v_{2} \nabla w_{2}-u_{2} v_{2} \nabla \sigma_{2}\left(v_{2}\right)\right]
\end{aligned}
$$

in $\Omega_{T}$, where $\Omega_{T}:=\Omega \times(0, T]$, and $\Omega$ is a bounded domain in $\mathbb{R}^{d}, d=1$ or 2 . Let $y$ be the vertical variable, with $y=0$ being the solid horizontal substrate. Then $u_{1}(x, t)$ and $w_{1}(x, t)$ are the height and reduced pressure, respectively, at $x \in \Omega$ and time $t$ of the lower liquid having viscosity $\mu>0$, whereas $u_{2}(x, t)$ and $w_{2}(x, t)$ are the height and reduced pressure of the upper liquid having unit viscosity. The concentration of insoluble surfactant at the liquid-liquid interface, $y=u_{1}(x, t)$, is $v_{1}(x, t)$; and at the liquid-air interface, $y=\left(u_{1}+u_{2}\right)(x, t)$, is $v_{2}(x, t)$; see Figure 1. The constants $\rho_{i}, c_{i} \in \mathbb{R}_{>0}$ are the inverses of the surface Peclet numbers and the modified capillary numbers, respectively, with $i=1$ for the $y=u_{1}$ interface and $i=2$ for the $y=u_{1}+u_{2}$ interface. In addition, $\sigma_{i} \in C^{1}\left(\mathbb{R}_{\geq 0}\right)$ with $\sigma_{i}(s) \geq 0$ and $\sigma_{i}^{\prime}(s)<0$ for all $s \in \mathbb{R}_{\geq 0}$ is the constitutive equation of state relating the surface tension $\sigma_{i}$ to $v_{i}$ on the $i$ th interface, i.e. surfactant reduces surface tension. An empirical model, proposed in [11], often used in the literature is $\sigma_{i}(s):=\left(\alpha_{i}+1\right)\left[1+\theta\left(\alpha_{i}\right) s\right]^{-3}-\alpha_{i}$, where $\theta\left(\alpha_{i}\right):=\left(\frac{\alpha_{i}+1}{\alpha_{i}}\right)^{\frac{1}{3}}-1$ and $\alpha_{i} \in \mathbb{R}_{>0}$ relates to the activity of the surfactant. Hence $\sigma_{i}:[0,1] \rightarrow[0,1]$. We shall assume that the surfactant concentration for each interface is dilute, $v_{i} \in[0,1]$, in which case the limit $\alpha_{i} \rightarrow \infty$ is taken, and the equation of state simplifies to

$$
\sigma_{i}(s) \equiv \sigma(s):=1-s \quad i=1,2 .
$$

The van der Waals forces, $\phi_{j}, j=1 \rightarrow 3$ in $(1.1 \mathrm{c}, \mathrm{d})$ acting simultaneously on the three heights, are given by

$$
\phi_{j}(s)=\phi_{j}^{+}(s)+\phi_{j}^{-}(s), \quad \phi_{j}^{+}(s):=-\delta_{j} s^{-\nu_{j}}, \quad \nu_{j}>3, \quad \phi_{j}^{-}(s):=a_{j} s^{-3},
$$


where $a_{j} \in \mathbb{R}_{\geq 0}$ is a scaled dimensionless Hamaker constant and $\delta_{j} \in \mathbb{R}_{\geq 0}$ represents the effect of repulsive van der Waals forces. We shall assume throughout that $\delta_{i}>0, i=1,2$, so that these repulsive forces prevent both films from rupturing, i.e. $u_{i}>0$. However, there is no a priori bound below on $u_{i}$ so $(1.1 \mathrm{a}-\mathrm{f})$ is a degenerate nonlinear parabolic system, which is fourth order in $u_{i}$. This degeneracy makes the analysis/numerical analysis of the system particularly difficult. In addition, as there is no maximum principle for parabolic equations of fourth order, a naive discretization does not guarantee the nonnegativity of the approximation to $u_{i}$.

In [1] a finite element approximation to the single-layered surfactant model in presence of van der Waals forces, $(1.1 \mathrm{a}-\mathrm{f})$ with $c_{1}=0, \mu=1, v_{1}(\cdot, 0)=0$ and $\phi_{1} \equiv \phi_{2} \equiv 0$, was presented. In addition, convergence of this approximation was proved, yielding an existence proof for the degenerate nonlinear parabolic system. It is the aim of this paper to adapt the techniques in [1] to present, and prove convergence of, a finite element approximation to $(1.1 \mathrm{a}-\mathrm{f})$.

As remarked previously, recall (1.2), the physically relevant values of $v_{i}$ lie in the interval $[0,1]$. Noting this, it is convenient for the analysis is this paper, as it was in [1,2], to replace $v_{i}$ in non-differentiated terms of $v_{i}$ by $\beta^{1}\left(v_{i}\right)$; where for a given $M \geq 1, \beta^{M}: \mathbb{R} \rightarrow(-\infty, M]$ is defined as

$$
\beta^{M}(s)=[s-M]_{-}+M, \quad \text { with } \quad[s]_{-}=\min \{s, 0\} .
$$

This two-layered system introduces new difficulties, and it is also convenient in the case $d=2$ to replace $u_{1}$ in non-differentiated terms of $u_{1}$, which are not arguments of $\phi_{i}$, by $\beta^{M}\left(u_{1}\right)$ for some sufficiently large cut-off $M$. We will return to the need for these cut-offs later in this section.

Altogether, in this paper we consider the following initial boundary value problem:

(P) Find functions $\left\{u_{i}, v_{i}, w_{i}\right\}_{i=1}^{2}: \Omega \times[0, T] \rightarrow \mathbb{R}$ such that

$$
\begin{aligned}
& \mu \frac{\partial u_{1}}{\partial t}=\nabla \cdot\left[\frac{1}{3}\left[\beta^{M}\left(u_{1}\right)\right]^{3} \nabla w_{1}+\frac{1}{2}\left[\beta^{M}\left(u_{1}\right)\right]^{2} u_{2} \nabla w_{2}-\frac{1}{2}\left[\beta^{M}\left(u_{1}\right)\right]^{2} \nabla\left(\sigma\left(v_{1}\right)+\sigma\left(v_{2}\right)\right)\right] \quad \text { in } \Omega_{T}, \\
& \mu \frac{\partial u_{2}}{\partial t}=\nabla \cdot\left[\frac{1}{2}\left[\beta^{M}\left(u_{1}\right)\right]^{2} u_{2} \nabla w_{1}+\beta^{M}\left(u_{1}\right) u_{2}^{2} \nabla w_{2}-\beta^{M}\left(u_{1}\right) u_{2} \nabla\left(\sigma\left(v_{1}\right)+\sigma\left(v_{2}\right)\right)\right] \\
& +\mu \nabla \cdot\left[\frac{1}{3} u_{2}^{3} \nabla w_{2}-\frac{1}{2} u_{2}^{2} \nabla \sigma\left(v_{2}\right)\right] \quad \text { in } \Omega_{T}, \\
& w_{1}-w_{2}=-c_{1} \Delta u_{1}+\phi_{1}\left(u_{1}\right)-\phi_{2}\left(u_{2}\right) \\
& \text { in } \Omega_{T} \text {, } \\
& w_{2}=-c_{2} \Delta\left(u_{1}+u_{2}\right)+\phi_{2}\left(u_{2}\right)+\phi_{3}\left(u_{1}+u_{2}\right) \\
& \text { in } \Omega_{T} \text {, } \\
& \mu \frac{\partial v_{1}}{\partial t}=\rho_{1} \mu \Delta v_{1}+\nabla \cdot\left[\frac{1}{2}\left[\beta^{M}\left(u_{1}\right)\right]^{2} \beta^{1}\left(v_{1}\right) \nabla w_{1}+\beta^{M}\left(u_{1}\right) \beta^{1}\left(v_{1}\right)\left(u_{2} \nabla w_{2}-\nabla\left[\sigma\left(v_{1}\right)+\sigma\left(v_{2}\right)\right]\right)\right] \\
& \text { in } \Omega_{T} \\
& \mu \frac{\partial v_{2}}{\partial t}=\rho_{2} \mu \Delta v_{2}+\nabla \cdot\left[\frac{1}{2}\left[\beta^{M}\left(u_{1}\right)\right]^{2} \beta^{1}\left(v_{2}\right) \nabla w_{1}+\beta^{M}\left(u_{1}\right) \beta^{1}\left(v_{2}\right)\left(u_{2} \nabla w_{2}-\nabla\left[\sigma\left(v_{1}\right)+\sigma\left(v_{2}\right)\right]\right)\right] \\
& +\nabla \cdot\left[\frac{1}{2} u_{2}^{2} \beta^{1}\left(v_{2}\right) \nabla w_{2}-u_{2} \beta^{1}\left(v_{2}\right) \nabla \sigma\left(v_{2}\right)\right] \quad \text { in } \Omega_{T} \\
& u_{1}(x, 0)=u_{1}^{0}(x)>0, \quad v_{1}(x, 0)=v_{1}^{0}(x) \geq 0 \\
& \forall x \in \Omega,(1.5 \mathrm{~g}) \\
& u_{2}(x, 0)=u_{2}^{0}(x)>0, \quad v_{2}(x, 0)=v_{2}^{0}(x) \geq 0 \\
& \forall x \in \Omega
\end{aligned}
$$

with no flux boundary conditions on $(1.5 \mathrm{a}, \mathrm{b}),(1.5 \mathrm{e}, \mathrm{f})$, and homogeneous Neumann boundary conditions on $(1.5 \mathrm{c}, \mathrm{d})$. The latter can be interpreted as a $90^{\circ}$ angle condition on the film surfaces, where they meet the exterior container. In the above $\mu, c_{i}, \rho_{i} \in \mathbb{R}_{>0}$ are given constants, while $\sigma(\cdot), \phi_{j}(\cdot)$ and $\beta^{M}(\cdot)$ are given by (1.2), (1.3) and (1.4) with $a_{j}, \delta_{3} \in \mathbb{R}_{\geq 0}, \delta_{1}, \delta_{2} \in \mathbb{R}_{>0}$ and $M \geq 1$.

The basic ingredients of our approach are two energy bounds combined with a regularization procedure. In particular, for any given $\varepsilon \in(0,1)$, we introduce the regularized function

$$
\beta_{\varepsilon}^{M}(s):=\max \left\{\beta^{M}(s), \varepsilon\right\}
$$

with yields the regularised system $\left(\mathrm{P}_{\varepsilon}\right)$; that is, $(\mathrm{P})$ with $\left\{u_{i}, v_{i}, \beta^{1}\left(v_{i}\right), w_{i}\right\}_{i=1}^{2}$ replaced by $\left\{u_{i, \varepsilon}, v_{i, \varepsilon}, \beta_{\varepsilon}^{1}\left(v_{i, \varepsilon}\right)\right.$, $\left.w_{i, \varepsilon}\right\}_{i=1}^{2}$. On defining the horizontal velocity fields $\mathcal{V}_{i, \varepsilon}(x, t, y)$, where $y$ is the vertical variable - recall Figure 1 , 
we have from lubrication theory, similarly to $[1,2]$, that

$$
\mu \frac{\partial^{2} \mathcal{V}_{1, \varepsilon}}{\partial y^{2}}=\nabla w_{1, \varepsilon} \quad \text { in } \Omega_{T} \times\left(0, u_{1, \varepsilon}(x, t)\right), \quad \text { and } \quad \frac{\partial^{2} \mathcal{V}_{2, \varepsilon}}{\partial y^{2}}=\nabla w_{2, \varepsilon} \quad \text { in } \Omega_{T} \times\left(u_{1, \varepsilon}(x, t),\left(u_{1, \varepsilon}+u_{2, \varepsilon}\right)(x, t)\right)
$$

subject to the boundary conditions

$$
\begin{aligned}
& \mathcal{V}_{1, \varepsilon}(x, t, 0)=0, \quad \mu \frac{\partial \mathcal{V}_{1, \varepsilon}}{\partial y}\left(x, t, u_{1, \varepsilon}(x, t)\right)=\frac{\partial \mathcal{V}_{2, \varepsilon}}{\partial y}\left(x, t, u_{1, \varepsilon}(x, t)\right)+\nabla \sigma\left(v_{1, \varepsilon}(x, t)\right), \\
& \mathcal{V}_{1, \varepsilon}\left(x, t, u_{1, \varepsilon}(x, t)\right)=\mathcal{V}_{2, \varepsilon}\left(x, t, u_{1, \varepsilon}(x, t)\right), \quad \frac{\partial \mathcal{V}_{2, \varepsilon}}{\partial y}\left(x, t,\left(u_{1, \varepsilon}+u_{2, \varepsilon}\right)(x, t)\right)=\nabla \sigma\left(v_{2, \varepsilon}(x, t)\right) \quad \forall(x, t) \in \Omega_{T}, \\
& \left.\begin{array}{rl}
{\left[\mathcal{V}_{1, \varepsilon} \cdot \nu_{\partial \Omega}\right](x, t, y)=0} & \forall y \in\left[0, u_{1, \varepsilon}(x, t)\right], \\
{\left[\mathcal{V}_{2, \varepsilon} \cdot \nu_{\partial \Omega}\right](x, t, y)=0} & \forall y \in\left[u_{1, \varepsilon}(x, t),\left(u_{1, \varepsilon}+u_{2, \varepsilon}\right)(x, t)\right]
\end{array}\right\} \quad \forall(x, t) \in \partial \Omega \times(0, T) ; \quad(1.7 \mathrm{~b})
\end{aligned}
$$

where $\nu_{\partial \Omega}$ is normal to $\partial \Omega$, and $\nabla$, as throughout, is respect to the horizontal variable $x$, and not the vertical variable $y$. The above yields for any $(x, t) \in \Omega_{T}$ that

$$
\begin{aligned}
& \mathcal{V}_{1, \varepsilon}(x, t, y)=-\frac{y}{\mu} \sum_{i=1}^{2}\left[u_{i, \varepsilon}(x, t) \nabla w_{i, \varepsilon}(x, t)-\nabla \sigma\left(v_{i, \varepsilon}(x, t)\right)\right]+\frac{y^{2}}{\mu} \nabla w_{1, \varepsilon}(x, t) \quad \text { for } y \in\left[0, u_{1, \varepsilon}(x, t)\right], \\
& \mathcal{V}_{2, \varepsilon}(x, t, y)=\mathcal{V}_{1, \varepsilon}\left(x, t, u_{1, \varepsilon}(x, t)\right)+\left(y-u_{1, \varepsilon}(x, t)\right)\left\{\left[\frac{y-u_{1, \varepsilon}(x, t)}{2}-u_{2, \varepsilon}(x, t)\right] \nabla w_{2, \varepsilon}(x, t)+\nabla \sigma\left(v_{2, \varepsilon}(x, t)\right)\right\} \\
& \text { for } y \in\left[u_{1, \varepsilon}(x, t),\left(u_{1, \varepsilon}+u_{2, \varepsilon}\right)(x, t)\right] .
\end{aligned}
$$

We can then recast the corresponding $\left(\mathrm{P}_{\varepsilon}\right)$ versions of $(1.5 \mathrm{a}, \mathrm{b})$ and $(1.5 \mathrm{e}, \mathrm{f})$, with $\beta^{M}\left(u_{1, \varepsilon}\right)$ replaced by $u_{1, \varepsilon}$, as

$$
\begin{aligned}
& \frac{\partial u_{1, \varepsilon}}{\partial t}+\nabla \cdot\left(\int_{0}^{u_{1, \varepsilon}} \mathcal{V}_{1, \varepsilon}(\cdot, \cdot, y) \mathrm{d} y\right)=0, \quad \frac{\partial u_{2, \varepsilon}}{\partial t}+\nabla \cdot\left(\int_{u_{1, \varepsilon}}^{u_{2, \varepsilon}} \mathcal{V}_{2, \varepsilon}(\cdot, \cdot, y) \mathrm{d} y\right)=0 \quad \text { in } \Omega_{T}, \\
& \frac{\partial v_{1, \varepsilon}}{\partial t}+\nabla \cdot\left(\mathcal{V}_{1, \varepsilon}\left(\cdot, \cdot, u_{1, \varepsilon}\right) \beta_{\varepsilon}^{1}\left(v_{1, \varepsilon}\right)\right)=\rho_{1} \Delta v_{1, \varepsilon}, \quad \frac{\partial v_{2, \varepsilon}}{\partial t}+\nabla \cdot\left(\mathcal{V}_{2, \varepsilon}\left(\cdot, \cdot, u_{1, \varepsilon}+u_{2, \varepsilon}\right) \beta_{\varepsilon}^{1}\left(v_{2, \varepsilon}\right)\right)=\rho_{2} \Delta v_{2, \varepsilon}
\end{aligned}
$$

In order to derive the crucial energy bounds, we introduce

$$
F_{\varepsilon}^{\prime \prime}(s)=\left[\beta_{\varepsilon}^{1}(s)\right]^{-1} \quad \text { and } \quad F_{\varepsilon}(1)=F_{\varepsilon}^{\prime}(1)=0,
$$

which, on recalling (1.6), implies that

$$
F_{\varepsilon}(s):= \begin{cases}\frac{s^{2}-\varepsilon^{2}}{2 \varepsilon}+(\ln \varepsilon-1) s+1 & s \leq \varepsilon \\ s(\ln s-1)+1 & \varepsilon \leq s \leq 1 \\ \frac{1}{2}(s-1)^{2} & 1 \leq s\end{cases}
$$

Hence $F_{\varepsilon} \in C^{2,1}(\mathbb{R})$, and for later purposes, we note that

$$
F_{\varepsilon}(s) \geq \frac{s^{2}}{4}-\frac{1}{2} \quad \forall s \geq 0 \quad \text { and } \quad F_{\varepsilon}(s) \geq \frac{s^{2}}{2 \varepsilon} \quad \forall s \leq 0 ;
$$

see e.g. (2.4) in [2]. 
We will now derive several formal bounds for $\left\{u_{i, \varepsilon}, v_{i, \varepsilon}, w_{i, \varepsilon}\right\}_{i=1}^{2}$. Testing the $u_{i, \varepsilon}$ equation in (1.9a) with $w_{i, \varepsilon}, i=1,2$, combining with the $\left(\mathrm{P}_{\varepsilon}\right)$ versions of $(1.5 \mathrm{c}, \mathrm{d})$ and noting $(1.7 \mathrm{a}, \mathrm{b})$ yields that

$$
\begin{aligned}
\frac{\mathrm{d}}{\mathrm{d} t} \int_{\Omega} & {\left[\frac{c_{1}}{2}\left|\nabla u_{1, \varepsilon}\right|^{2}+\frac{c_{2}}{2}\left|\nabla\left(u_{1, \varepsilon}+u_{2, \varepsilon}\right)\right|^{2}+\left[\sum_{i=1}^{2} \Phi_{i}\left(u_{i, \varepsilon}\right)\right]+\Phi_{3}\left(u_{1, \varepsilon}+u_{2, \varepsilon}\right)\right] \mathrm{d} x+\mu \int_{\Omega}\left(\int_{0}^{u_{1, \varepsilon}}\left|\frac{\partial \mathcal{V}_{1, \varepsilon}}{\partial y}\right|^{2} \mathrm{~d} y\right) \mathrm{d} x } \\
& +\int_{\Omega}\left(\int_{u_{1, \varepsilon}}^{u_{1, \varepsilon}+u_{2, \varepsilon}}\left|\frac{\partial \mathcal{V}_{2, \varepsilon}}{\partial y}\right|^{2} \mathrm{~d} y\right) \mathrm{d} x=\int_{\Omega}\left[\mathcal{V}_{1, \varepsilon}\left(\cdot, \cdot, u_{1, \varepsilon}\right) \nabla \sigma\left(v_{1, \varepsilon}\right)+\mathcal{V}_{2, \varepsilon}\left(\cdot, \cdot, u_{2, \varepsilon}\right) \nabla \sigma\left(v_{2, \varepsilon}\right)\right] \mathrm{d} x,
\end{aligned}
$$

where $\Phi_{j}(\cdot)$ is an antiderivative of $\phi_{j}(\cdot)$, i.e. $\Phi_{j}^{\prime}(\cdot) \equiv \phi_{j}(\cdot), j=1 \rightarrow 3$. Testing the $v_{i, \varepsilon}$ equation in (1.9b) with $F_{\varepsilon}^{\prime}\left(v_{i, \varepsilon}\right)$, noting $(1.10)$ and combining yields that

$$
\frac{\mathrm{d}}{\mathrm{d} t} \int_{\Omega} \sum_{i=1}^{2} F_{\varepsilon}\left(v_{i, \varepsilon}\right) \mathrm{d} x+\sum_{i=1}^{2} \rho_{i} \int_{\Omega} F_{\varepsilon}^{\prime \prime}\left(v_{i, \varepsilon}\right)\left|\nabla v_{i, \varepsilon}\right|^{2} \mathrm{~d} x=\int_{\Omega}\left[\mathcal{V}_{1, \varepsilon}\left(\cdot, \cdot, u_{1, \varepsilon}\right) \nabla v_{1, \varepsilon}+\mathcal{V}_{2, \varepsilon}\left(\cdot, \cdot, u_{2, \varepsilon}\right) \nabla v_{2, \varepsilon}\right] \mathrm{d} x
$$

Combining (1.13) and (1.14), and noting (1.2), yields the formal energy identity

$$
\begin{aligned}
& \frac{\mathrm{d}}{\mathrm{d} t} \int_{\Omega}\left[\frac{c_{1}}{2}\left|\nabla u_{1, \varepsilon}\right|^{2}+\frac{c_{2}}{2}\left|\nabla\left(u_{1, \varepsilon}+u_{2, \varepsilon}\right)\right|^{2}+\sum_{i=1}^{2}\left[\Phi_{i}\left(u_{i, \varepsilon}\right)+F_{\varepsilon}\left(v_{i, \varepsilon}\right)\right]+\Phi_{3}\left(u_{1, \varepsilon}+u_{2, \varepsilon}\right)\right] \mathrm{d} x \\
& +\mu \int_{\Omega}\left(\int_{0}^{u_{1, \varepsilon}}\left|\frac{\partial \mathcal{V}_{1, \varepsilon}}{\partial y}\right|^{2} \mathrm{~d} y\right) \mathrm{d} x+\int_{\Omega}\left(\int_{u_{1, \varepsilon}}^{u_{1, \varepsilon}+u_{2, \varepsilon}}\left|\frac{\partial \mathcal{V}_{2, \varepsilon}}{\partial y}\right|^{2} \mathrm{~d} y\right) \mathrm{d} x+\sum_{i=1}^{2} \rho_{i} \int_{\Omega} F_{\varepsilon}^{\prime \prime}\left(v_{i, \varepsilon}\right)\left|\nabla v_{i, \varepsilon}\right|^{2} \mathrm{~d} x=0 .
\end{aligned}
$$

Noting $(1.8 \mathrm{a}, \mathrm{b})$ and Young's inequality,

$$
|r s| \leq \frac{\gamma}{2} r^{2}+\frac{1}{2 \gamma} s^{2} \quad \forall r, s \in \mathbb{R}, \quad \gamma \in \mathbb{R}_{>0}
$$

one can derive the following inequalities for any $\gamma^{*} \in\left(\frac{1}{2}, \frac{2}{3}\right)$

$$
\begin{aligned}
& \mu \int_{0}^{u_{1, \varepsilon}}\left|\frac{\partial \mathcal{V}_{1, \varepsilon}}{\partial y}\right|^{2} \mathrm{~d} y=\frac{1}{\mu}\left[\frac{1}{3} u_{1, \varepsilon}^{3}\left|\nabla w_{1, \varepsilon}\right|^{2}+u_{1, \varepsilon}\left|u_{2, \varepsilon} \nabla w_{2, \varepsilon}-\nabla\left(\sigma\left(v_{1, \varepsilon}\right)+\sigma\left(v_{2, \varepsilon}\right)\right)\right|^{2}\right. \\
& \left.+u_{1, \varepsilon}^{2} \nabla w_{1, \varepsilon} \cdot\left[u_{2, \varepsilon} \nabla w_{2, \varepsilon}+\nabla\left(\sigma\left(v_{1, \varepsilon}\right)+\sigma\left(v_{2, \varepsilon}\right)\right)\right]\right] \\
& \geq \frac{1}{\mu}\left[\left(\frac{1}{3}-\frac{\gamma^{*}}{2}\right) u_{1, \varepsilon}^{3}\left|\nabla w_{1, \varepsilon}\right|^{2}+\left(1-\frac{1}{2 \gamma^{*}}\right) u_{1, \varepsilon}\left|u_{2, \varepsilon} \nabla w_{2, \varepsilon}-\nabla\left(\sigma\left(v_{1, \varepsilon}\right)+\sigma\left(v_{2, \varepsilon}\right)\right)\right|^{2}\right], \\
& \int_{u_{1, \varepsilon}}^{u_{1, \varepsilon}+u_{2, \varepsilon}}\left|\frac{\partial \mathcal{V}_{2, \varepsilon}}{\partial y}\right|^{2} \mathrm{~d} y=\frac{1}{3} u_{2, \varepsilon}^{3}\left|\nabla w_{2, \varepsilon}\right|^{2}+u_{2, \varepsilon}\left|\nabla \sigma\left(v_{2, \varepsilon}\right)\right|^{2}-u_{2, \varepsilon}^{2} \nabla w_{2, \varepsilon} \cdot \nabla \sigma\left(v_{2, \varepsilon}\right) \\
& \geq\left(\frac{1}{3}-\frac{\gamma^{*}}{2}\right) u_{2, \varepsilon}^{3}\left|\nabla w_{2, \varepsilon}\right|^{2}+\left(1-\frac{1}{2 \gamma^{*}}\right) u_{2, \varepsilon}\left|\nabla \sigma\left(v_{2, \varepsilon}\right)\right|^{2} .
\end{aligned}
$$

From (1.15), (1.17a,b), (1.10) and (1.4), one can derive uniform bounds on $\nabla u_{i, \varepsilon}$ in $L^{\infty}\left(0, T ; L^{2}(\Omega)\right)$ and $\nabla v_{i, \varepsilon}$ in $L^{2}\left(\Omega_{T}\right)$. We note the crucial role that the cut-off $\beta^{1}(\cdot)$ on $v_{i, \varepsilon}$ plays in the $v_{i, \varepsilon}$ bound, recall (1.10). Of course one could replace $\beta^{1}(\cdot)$ with $\beta^{M}(\cdot)$, where $M$ arbitrarily large. However, as it does not appear possible to obtain an a priori $L^{\infty}\left(\Omega_{T}\right)$ bound on $v_{i, \varepsilon}$, some cut-off above on $v_{i, \varepsilon}$ is required. In addition, the singularity in $\Phi_{i}, i=1,2$ at the origin yields the positivity of $u_{i, \varepsilon}$. Furthermore, the bound (1.12) together with (1.15) yields that $\int_{\Omega_{T}}\left[v_{i, \varepsilon}\right]_{-}^{2} \mathrm{~d} x \mathrm{~d} t \leq C \varepsilon$. As can be seen from the above, it is not necessary to have the cut-off $\beta^{M}(\cdot)$ 
on $u_{1, \varepsilon}$ in the coefficients in $\left(\mathrm{P}_{\varepsilon}\right)$, in order to obtain the formal energy identity (1.15). This cut-off on $u_{1, \varepsilon}$ in these coefficients is required for the second energy bound, see below; and this bound is only required if $d=2$. It is easily deduced, that the effect of this cut-off is just to modify the term (1.17a) in (1.15); that is, $u_{1, \varepsilon}$ is replace by $\beta^{M}\left(u_{1, \varepsilon}\right)$.

In order to obtain the second energy bound we define a function $G \in C^{\infty}\left(\mathbb{R}_{>0}\right)$ such that $\eta^{3} \nabla G^{\prime}(\eta)=\nabla \eta$; that is, for $s>0$

$$
G^{\prime \prime}(s)=s^{-3} \Rightarrow G^{\prime}(s)=-\frac{1}{2} s^{-2} \Rightarrow G(s)=\frac{1}{2} s^{-1}
$$

where the constants of integration have been chosen to be zero. In addition, we introduce $G_{M} \in C^{2}\left(\mathbb{R}_{>0}\right)$ such that $\left[\beta^{M}(\eta)\right]^{3} \nabla G_{M}^{\prime}(\eta)=\nabla \eta$; that is, for all $M \geq 1$ and $s>0$

$$
G_{M}^{\prime \prime}(s)=\left[\beta^{M}(s)\right]^{-3} \Rightarrow G_{M}(s)= \begin{cases}G(s) & s \in(0, M] \\ \frac{1}{2 M}\left(\left(\frac{s}{M}\right)^{2}-3\left(\frac{s}{M}\right)+3\right) & s \geq M .\end{cases}
$$

Testing the $\left(\mathrm{P}_{\varepsilon}\right)$ versions of $(1.5 \mathrm{a})$ with $G_{M}^{\prime}\left(u_{1, \varepsilon}\right)$, (1.5b) with $G^{\prime}\left(u_{2, \varepsilon}\right)$, (1.5c) with $-\Delta u_{1, \varepsilon}$ and $(1.5 \mathrm{~d})$ with $-\Delta u_{2, \varepsilon}$ and combining, formally yields, on noting (1.3), (1.18), (1.19) and the no flux boundary conditions, that

$$
\begin{aligned}
& \frac{\mathrm{d}}{\mathrm{d} t} \int_{\Omega}\left[\mu G_{M}\left(u_{1, \varepsilon}\right)+G\left(u_{2, \varepsilon}\right)\right] \mathrm{d} x+\frac{1}{3} \int_{\Omega}\left[c_{1}\left|\Delta u_{1, \varepsilon}\right|^{2}+c_{2}\left|\Delta\left(u_{1, \varepsilon}+u_{2, \varepsilon}\right)\right|^{2}\right] \mathrm{d} x \\
& +\sum_{i=1}^{2} \int_{\Omega}\left(\phi_{i}^{+}\right)^{\prime}\left(u_{i, \varepsilon}\right)\left|\nabla u_{i, \varepsilon}\right|^{2} \mathrm{~d} x+\int_{\Omega}\left(\phi_{3}^{+}\right)^{\prime}\left(u_{1, \varepsilon}+u_{2, \varepsilon}\right)\left|\nabla\left(u_{1, \varepsilon}+u_{2, \varepsilon}\right)\right|^{2} \mathrm{~d} x \\
& =-\int_{\Omega}\left(u_{2, \varepsilon} \nabla w_{2, \varepsilon}-\nabla\left[\sigma\left(v_{1, \varepsilon}\right)+\sigma\left(v_{2, \varepsilon}\right)\right]\right) \cdot\left(\frac{1}{2}\left[\beta^{M}\left(u_{1, \varepsilon}\right)\right]^{-1} \nabla u_{1, \varepsilon}+\frac{1}{\mu} \beta^{M}\left(u_{1, \varepsilon}\right) u_{2, \varepsilon}^{-2} \nabla u_{2, \varepsilon}\right) \mathrm{d} x \\
& \quad-\frac{1}{2 \mu} \int_{\Omega}\left[\beta^{M}\left(u_{1, \varepsilon}\right)\right]^{2} \nabla w_{1, \varepsilon} \cdot u_{2, \varepsilon}^{-2} \nabla u_{2, \varepsilon} \mathrm{d} x+\frac{1}{2} \int_{\Omega} u_{2, \varepsilon}^{-1} \nabla\left[\sigma\left(v_{2, \varepsilon}\right)\right] \cdot \nabla u_{2, \varepsilon} \mathrm{d} x \\
& \quad-\sum_{i=1}^{2} \int_{\Omega}\left(\phi_{i}^{-}\right)^{\prime}\left(u_{i, \varepsilon}\right)\left|\nabla u_{i, \varepsilon}\right|^{2} \mathrm{~d} x-\int_{\Omega}\left(\phi_{3}^{-}\right)^{\prime}\left(\sum_{i=1}^{2} u_{i, \varepsilon}\right)\left|\nabla\left(\sum_{i=1}^{2} u_{i, \varepsilon}\right)\right|^{2} \mathrm{~d} x .
\end{aligned}
$$

It follows from $(1.20),(1.16),(1.4),(1.3)$ and the bound

$$
s^{-\alpha} \leq\left[\beta^{M}(s)\right]^{-\alpha} \leq \gamma s^{-\zeta}+C(\gamma, \alpha, \zeta, M) \quad \forall s, \gamma \in \mathbb{R}_{>0}, \quad \alpha \in(0, \zeta),
$$

for $\zeta=\nu_{j}+1, j=1,2$, and for both $\alpha=3$ and 4 , that

$$
\begin{aligned}
& \frac{\mathrm{d}}{\mathrm{d} t} \int_{\Omega}\left[\mu G_{M}\left(u_{1, \varepsilon}\right)+G\left(u_{2, \varepsilon}\right)\right] \mathrm{d} x+\frac{1}{3} \int_{\Omega}\left[c_{1}\left|\Delta u_{1, \varepsilon}\right|^{2}+c_{2}\left|\Delta\left(u_{1, \varepsilon}+u_{2, \varepsilon}\right)\right|^{2}\right] \mathrm{d} x \\
& +\sum_{i=1}^{2} \int_{\Omega}\left(\phi_{i}^{+}\right)^{\prime}\left(u_{i, \varepsilon}\right)\left|\nabla u_{i, \varepsilon}\right|^{2} \mathrm{~d} x+\int_{\Omega}\left(\phi_{3}^{+}\right)^{\prime}\left(u_{1, \varepsilon}+u_{2, \varepsilon}\right)\left|\nabla\left(u_{1, \varepsilon}+u_{2, \varepsilon}\right)\right|^{2} \mathrm{~d} x \\
& \leq C\left[\int_{\Omega} \beta^{M}\left(u_{1, \varepsilon}\right)\left|u_{2, \varepsilon} \nabla w_{2, \varepsilon}-\nabla\left[\sigma\left(v_{1, \varepsilon}\right)+\sigma\left(v_{2, \varepsilon}\right)\right]\right|^{2} \mathrm{~d} x+\int_{\Omega} u_{2, \varepsilon}\left|\nabla\left[\sigma\left(v_{2, \varepsilon}\right)\right]\right|^{2} \mathrm{~d} x\right. \\
& \left.\quad+\int_{\Omega}\left[\beta^{M}\left(u_{1, \varepsilon}\right)\right]^{3}\left|\nabla w_{1, \varepsilon}\right|^{2} \mathrm{~d} x+\sum_{i=1}^{2} \int_{\Omega}\left|\nabla u_{i, \varepsilon}\right|^{2} \mathrm{~d} x\right] .
\end{aligned}
$$

From $(1.22),(1.15)$, and $(1.17 \mathrm{a}, \mathrm{b})$ with $u_{1, \varepsilon}$ replaced by $\beta^{M}\left(u_{1, \varepsilon}\right)$, one obtains that $u_{i, \varepsilon}$ is uniformly bounded in $L^{2}\left(0, T ; H^{2}(\Omega)\right)$. We note that we have used the cut-off on $u_{1, \varepsilon}$, in order to control the first and second terms on the right hand side of (1.20). 
It is the goal of this paper to derive a finite element method that is consistent with the formal energy bounds (1.15) and (1.22).

This paper is organized as follows. In Section 2 we formulate a fully practical finite element approximation of the degenerate problem $(\mathrm{P})$ and derive discrete analogues of the energy bounds $(1.15)$, and $(1.22)$ if $d=2$ and $\nu_{j} \geq 7, j=1,2$, in (1.3). In Section 3 we prove convergence, and hence existence of a solution to the system $(\mathrm{P})$. In the case $d=1$, we prove existence of a solution to $(\mathrm{P})$ with $\beta^{M}\left(u_{1}\right)$ replaced by $u_{1}$.

Finally, although there is a vast amount of work in the applied mathematics, physics and engineering literature, there is very little work in the PDE literature on surfactant type problems. To our knowledge, there is no work on the two-layered system $(\mathrm{P})$. For the single-layered system, the only papers that we are aware of are the following. A local existence result without cut-offs is shown in [8] for the pure initial-value problem with very smooth initial data. A global existence result in one space dimension without van der Waals forces and cut-offs can be found in [5], but this result does not allow for $\sigma$ of the form (1.2). A global existence result, via the convergence of a finite element approximation, in both one and two space dimensions with van der Waals forces and with a cut-off on the surfactant concentration in the coefficients can be found in [1]. The above results are all for the case of an insoluble surfactant. An extension of the existence result in [1] to the case of a soluble surfactant can be found in [3]. Of course, it is possible to extend the results in this paper to the more complicated two-layered case in the presence of soluble surfactants by combining the ideas here with those in [3].

\section{Notation and auxiliary results}

Let $D \subset \mathbb{R}^{d}, d=1$ or 2 , with a Lipschitz boundary $\partial D$ if $d=2$. We adopt the standard notation for Sobolev spaces, denoting the norm of $W^{m, q}(D)(m \in \mathbb{N}, q \in[1, \infty])$ by $\|\cdot\|_{m, q, D}$ and the semi-norm by $|\cdot|_{m, q, D}$. We extend these norms and semi-norms in the natural way to the corresponding spaces of vector and matrix valued functions. For $q=2, W^{m, 2}(D)$ will be denoted by $H^{m}(D)$ with the associated norm and semi-norm written as, respectively, $\|\cdot\|_{m, D}$ and $|\cdot|_{m, D}$. For notational convenience, we drop the domain subscript on the above norms and semi-norms in the case $D \equiv \Omega$. Throughout $(\cdot, \cdot)$ denotes the standard $L^{2}$ inner product over $\Omega$, while $q^{\prime}$ denotes for any $q \in[1, \infty]$ the "dual exponent" such that $\frac{1}{q}+\frac{1}{q^{\prime}}=1$. In addition we define

$$
f \eta:=\frac{1}{\underline{m}(\Omega)} \int_{\Omega} \eta \mathrm{d} x \quad \forall \eta \in L^{1}(\Omega),
$$

where $\underline{m}(D)$ denotes the measure of $D$.

It is convenient to introduce the "inverse Laplacian" operator $\mathcal{G}: \mathcal{F} \rightarrow Z$ such that

$$
(\nabla \mathcal{G} z, \nabla \eta)=\langle z, \eta\rangle_{q^{\prime}} \quad \forall \eta \in W^{1, q^{\prime}}(\Omega)
$$

where $\mathcal{F}:=\left\{z \in\left(W^{1, q^{\prime}}(\Omega)\right)^{\prime}:\langle z, 1\rangle_{q^{\prime}}=0\right\}$ and $Z:=\left\{z \in W^{1, q}(\Omega):(z, 1)=0\right\}$. Here and throughout $\langle\cdot, \cdot\rangle_{q^{\prime}}$ denotes the duality pairing between $\left(W^{1, q^{\prime}}(\Omega)\right)^{\prime}$ and $W^{1, q^{\prime}}(\Omega)$ for any $q \in(1,2]$. The well-posedness of $\mathcal{G}$ follows from the generalised Lax-Milgram theorem and the Poincaré inequality

$$
|\eta|_{0, r} \leq C\left(|\eta|_{1, r}+|(\eta, 1)|\right) \quad \forall \eta \in W^{1, r}(\Omega) \quad \text { and } \quad r \in[1, \infty]
$$

Throughout $C$ denotes a generic constant independent of $h, \tau$ and $\varepsilon$; the mesh and temporal discretization parameters and the regularization parameter. In addition $C\left(a_{1}, \ldots, a_{I}\right)$ denotes a constant depending on the arguments $\left\{a_{i}\right\}_{i=1}^{I}$. Furthermore $\cdot_{(\star)}$ denotes an expression with or without the subscript $\star$; similarly for superscripts. 


\section{FINITE ELEMENT APPROXIMATION}

We consider the finite element approximation of $(\mathrm{P})$ under the following assumptions on the mesh:

(A) Let $\Omega$ be a convex polygonal domain if $d=2$. Let $\left\{\mathcal{T}^{h}\right\}_{h>0}$ be a quasi-uniform family of partitionings of $\Omega$ into disjoint open simplices $\kappa$ with $h_{\kappa}:=\operatorname{diam}(\kappa)$ and $h:=\max _{\kappa \in \mathcal{T}^{h}} h_{\kappa}$, so that $\bar{\Omega}=\cup_{\kappa \in \mathcal{T}^{h}} \bar{\kappa}$. In addition, it is assumed for $d=2$ that all simplices $\kappa \in \mathcal{T}^{h}$ are right-angled.

We note that the right-angled simplices assumption is not a severe constraint, as there exist adaptive finite element codes that satisfy this requirement, see e.g. [10].

Associated with $\mathcal{T}^{h}$ is the finite element space

$$
S^{h}:=\left\{\chi \in C(\bar{\Omega}):\left.\chi\right|_{\kappa} \text { is linear } \forall \kappa \in \mathcal{T}^{h}\right\} \subset H^{1}(\Omega) .
$$

We introduce also

$$
S_{\geq 0}^{h}:=\left\{\chi \in S^{h}: \chi \geq 0 \text { in } \Omega\right\} \subset H_{\geq 0}^{1}(\Omega):=\left\{\eta \in H^{1}(\Omega): \eta \geq 0 \text { a.e. in } \Omega\right\},
$$

and similarly $S_{>0}^{h}$ and $H_{>0}^{1}(\Omega)$. Let $J$ be the set of nodes of $\mathcal{T}^{h}$ and $\left\{p_{j}\right\}_{j \in J}$ the coordinates of these nodes. Let $\left\{\chi_{j}\right\}_{j \in J}$ be the standard basis functions for $S^{h}$; that is $\chi_{j} \in S_{\geq 0}^{h}$ and $\chi_{j}\left(p_{i}\right)=\delta_{i j}$ for all $i, j \in J$. We introduce $\pi^{h}: C(\bar{\Omega}) \rightarrow S^{h}$, the interpolation operator, such that $\left(\pi^{h} \eta\right)\left(p_{j}\right)=\eta\left(p_{j}\right)$ for all $j \in J$. A discrete semi-inner product on $C(\bar{\Omega})$ is then defined by

$$
\left(\eta_{1}, \eta_{2}\right)^{h}:=\int_{\Omega} \pi^{h}\left(\eta_{1}(x) \eta_{2}(x)\right) \mathrm{d} x=\sum_{j \in J} m_{j} \eta_{1}\left(p_{j}\right) \eta_{2}\left(p_{j}\right)
$$

where $m_{j}:=\left(1, \chi_{j}\right)>0$. The induced discrete semi-norm is then $|\eta|_{h}:=\left[(\eta, \eta)^{h}\right]^{\frac{1}{2}}$, where $\eta \in C(\bar{\Omega})$. We introduce also the $L^{2}$ projection $Q^{h}: L^{2}(\Omega) \rightarrow S^{h}$ defined by

$$
\left(Q^{h} \eta, \chi\right)^{h}=(\eta, \chi) \quad \forall \chi \in S^{h} .
$$

Similarly to the approach in $[7,12]$ for the thin film equation, i.e. a single-layered system without surfactant, we introduce matrices $\Lambda_{\varepsilon}: S^{h} \rightarrow\left[L^{\infty}(\Omega)\right]^{d \times d}$, and $\Xi_{(M)}: S_{>0}^{h} \rightarrow\left[L^{\infty}(\Omega)\right]^{d \times d}$ such that for all $z^{h} \in S^{h}, \chi \in S_{>0}^{h}$ and a.e. in $\Omega$

$$
\begin{aligned}
& \Lambda_{\varepsilon}\left(z^{h}\right), \Xi_{(M)}(\chi) \text { are symmetric and positive semi-definite, } \\
& \Lambda_{\varepsilon}\left(z^{h}\right) \nabla \pi^{h}\left[F_{\varepsilon}^{\prime}\left(z^{h}\right)\right]=\nabla z^{h}, \quad\left[\Xi_{(M)}(\chi)\right]^{3} \nabla \pi^{h}\left[G_{(M)}^{\prime}(\chi)\right]=\nabla \chi .
\end{aligned}
$$

The construction of $\Xi$ and $\Lambda_{\varepsilon}$ is given in [1]. The construction of $\Xi_{M}$ is the same as that of $\Xi$, but with $G$ replaced by $G_{M}$. We note that the right-angle constraint on the partitioning $\mathcal{T}^{h}$ is exploited for these constructions. Throughout this paper we make use of the fact that the matrices $\Xi(\chi), \Xi_{M}\left(\eta^{h}\right)$ and $\Lambda_{\varepsilon}\left(z^{h}\right)$ commute with each other for any $\chi, \eta^{h} \in S_{>0}^{h}$ and $z^{h} \in S^{h}$. 
In addition to $\mathcal{T}^{h}$, let $\tau=\frac{T}{N}$ be the uniform time step and $t_{n}:=n \tau, n=0 \rightarrow N$. For any given $\varepsilon \in(0,1)$, we then consider the following fully practical finite element approximation of $(\mathrm{P})$ with $\sigma$ given by $(1.2)$, and $\phi_{j}$ given by (1.3):

$\left(\mathbf{P}_{\varepsilon}^{h, \tau}\right)$ For $n \geq 1$ find $\left\{\left\{U_{i, \varepsilon}^{n}, W_{i, \varepsilon}^{n}, V_{i, \varepsilon}^{n}\right\}_{i=1}^{2}\right\} \in\left[S^{h}\right]^{6}$ such that for all $\chi \in S^{h}$

$$
\begin{aligned}
& \mu\left(\frac{U_{1, \varepsilon}^{n}-U_{1, \varepsilon}^{n-1}}{\tau}, \chi\right)^{h}+\frac{1}{3}\left(\left[\Xi_{M}\left(U_{1, \varepsilon}^{n}\right)\right]^{3} \nabla W_{1, \varepsilon}^{n}, \nabla \chi\right)+\frac{1}{2}\left(\left[\Xi_{M}\left(U_{1, \varepsilon}^{n}\right)\right]^{2} \Xi\left(U_{2, \varepsilon}^{n}\right) \nabla W_{2, \varepsilon}^{n}, \nabla \chi\right) \\
& =-\frac{1}{2}\left(\left[\Xi_{M}\left(U_{1, \varepsilon}^{n}\right)\right]^{\frac{3}{2}}\left[\Xi_{M}\left(U_{1, \varepsilon}^{n-1}\right)\right]^{\frac{1}{2}} \nabla\left[V_{1, \varepsilon}^{n-1}+V_{2, \varepsilon}^{n-1}\right], \nabla \chi\right), \\
& \mu\left(\frac{U_{2, \varepsilon}^{n}-U_{2, \varepsilon}^{n-1}}{\tau}, \chi\right)^{h}+\left(\left[\frac{\mu}{3}\left[\Xi\left(U_{2, \varepsilon}^{n}\right)\right]^{3}+\Xi_{M}\left(U_{1, \varepsilon}^{n}\right)\left[\Xi\left(U_{2, \varepsilon}^{n}\right)\right]^{2}\right] \nabla W_{2, \varepsilon}^{n}, \nabla \chi\right)+\frac{1}{2}\left(\left[\Xi_{M}\left(U_{1, \varepsilon}^{n}\right)\right]^{2} \Xi\left(U_{2, \varepsilon}^{n}\right) \nabla W_{1, \varepsilon}^{n}, \nabla \chi\right) \\
& =-\frac{\mu}{2}\left(\left[\Xi\left(U_{2, \varepsilon}^{n}\right)\right]^{\frac{3}{2}}\left[\Xi\left(U_{2, \varepsilon}^{n-1}\right)\right]^{\frac{1}{2}} \nabla V_{2, \varepsilon}^{n-1}, \nabla \chi\right) \\
& -\left(\left[\Xi_{M}\left(U_{1, \varepsilon}^{n}\right)\right]^{\frac{1}{2}}\left[\Xi_{M}\left(U_{1, \varepsilon}^{n-1}\right)\right]^{\frac{1}{2}} \Xi\left(U_{2, \varepsilon}^{n}\right) \nabla\left[V_{1, \varepsilon}^{n-1}+V_{2, \varepsilon}^{n-1}\right], \nabla \chi\right), \\
& c_{1}\left(\nabla U_{1, \varepsilon}^{n}, \nabla \chi\right)+\left(\phi_{1}^{+}\left(U_{1, \varepsilon}^{n}\right)+\phi_{1}^{-}\left(U_{1, \varepsilon}^{n-1}\right), \chi\right)^{h}-\left(\phi_{2}^{+}\left(U_{2, \varepsilon}^{n}\right)+\phi_{2}^{-}\left(U_{2, \varepsilon}^{n-1}\right), \chi\right)^{h}=\left(W_{1, \varepsilon}^{n}-W_{2, \varepsilon}^{n}, \chi\right)^{h}, \\
& c_{2}\left(\nabla\left[U_{1, \varepsilon}^{n}+U_{2, \varepsilon}^{n}\right], \nabla \chi\right)+\left(\phi_{2}^{+}\left(U_{2, \varepsilon}^{n}\right)+\phi_{2}^{-}\left(U_{2, \varepsilon}^{n-1}\right)+\phi_{3}^{+}\left(\sum_{i=1}^{2} U_{i, \varepsilon}^{n}\right)+\phi_{3}^{-}\left(\sum_{i=1}^{2} U_{i, \varepsilon}^{n-1}\right), \chi\right)^{h}=\left(W_{2, \varepsilon}^{n}, \chi\right)^{h} \\
& \mu\left(\frac{V_{1, \varepsilon}^{n}-V_{1, \varepsilon}^{n-1}}{\tau}, \chi\right)^{h}+\rho_{1} \mu\left(\nabla V_{1, \varepsilon}^{n}, \nabla \chi\right)+\left(\Xi_{M}\left(U_{1, \varepsilon}^{n}\right) \Lambda_{\varepsilon}\left(V_{1, \varepsilon}^{n}\right) \nabla\left[V_{1, \varepsilon}^{n}+V_{2, \varepsilon}^{n}\right], \nabla \chi\right) \\
& +\left(\Xi_{M}\left(U_{1, \varepsilon}^{n}\right) \Xi\left(U_{2, \varepsilon}^{n}\right) \Lambda_{\varepsilon}\left(V_{1, \varepsilon}^{n}\right) \nabla W_{2, \varepsilon}^{n}, \nabla \chi\right)=-\frac{1}{2}\left(\left[\Xi_{M}\left(U_{1, \varepsilon}^{n}\right)\right]^{2} \Lambda_{\varepsilon}\left(V_{1, \varepsilon}^{n}\right) \nabla W_{1, \varepsilon}^{n}, \nabla \chi\right), \\
& \mu\left(\frac{V_{2, \varepsilon}^{n}-V_{2, \varepsilon}^{n-1}}{\tau}, \chi\right)^{h}+\rho_{2} \mu\left(\nabla V_{2, \varepsilon}^{n}, \nabla \chi\right)+\left(\Xi_{M}\left(U_{1, \varepsilon}^{n}\right) \Lambda_{\varepsilon}\left(V_{2, \varepsilon}^{n}\right) \nabla\left[V_{1, \varepsilon}^{n}+V_{2, \varepsilon}^{n}\right], \nabla \chi\right) \\
& +\left(\Xi_{M}\left(U_{1, \varepsilon}^{n}\right) \Xi\left(U_{2, \varepsilon}^{n}\right) \Lambda_{\varepsilon}\left(V_{2, \varepsilon}^{n}\right) \nabla W_{2, \varepsilon}^{n}, \nabla \chi\right)+\frac{\mu}{2}\left(\left[\Xi\left(U_{2, \varepsilon}^{n}\right)\right]^{2} \Lambda_{\varepsilon}\left(V_{2, \varepsilon}^{n}\right) \nabla W_{2, \varepsilon}^{n}, \nabla \chi\right) \\
& +\mu\left(\Xi\left(U_{2, \varepsilon}^{n}\right) \Lambda_{\varepsilon}\left(V_{2, \varepsilon}^{n}\right) \nabla V_{2, \varepsilon}^{n}, \nabla \chi\right)=-\frac{1}{2}\left(\left[\Xi_{M}\left(U_{1, \varepsilon}^{n}\right)\right]^{2} \Lambda_{\varepsilon}\left(V_{2, \varepsilon}^{n}\right) \nabla W_{1, \varepsilon}^{n}, \nabla \chi\right)
\end{aligned}
$$

where, for $i=1,2, U_{i, \varepsilon}^{0} \in S_{>0}^{h}$ and $V_{i, \varepsilon}^{0} \in S^{h}$ are approximations of $u_{i}^{0}$ and $v_{i}^{0}$, respectively, e.g. $U_{i, \varepsilon}^{0} \equiv \pi^{h} u_{i}^{0}$ or $Q^{h} u_{i}^{0}$ and similarly for $V_{i, \varepsilon}^{0}$.

Remark 2.1. We note that the above system decouples into $(2.4 \mathrm{a}-\mathrm{d})$ and $(2.4 \mathrm{e}, \mathrm{f})$; that is, one updates the heights and pressures at the new time level, then the surfactant concentrations. $\left(\mathrm{P}_{\varepsilon}^{h, \tau}\right)$ is the natural extension of the approximation of the insoluble single-layered surfactant system studied in [1]. In particular, on setting $c_{1}=0, \phi_{1} \equiv \phi_{2} \equiv 0$ yields that $W_{\varepsilon}^{n} \equiv W_{1, \varepsilon}^{n} \equiv W_{2, \varepsilon}^{n}$ and $U_{\varepsilon}^{n} \equiv U_{1, \varepsilon}^{n}+U_{2, \varepsilon}^{n}, n=1 \rightarrow N$. Moreover, $\mu=1$ and $v_{1}^{0} \equiv 0$ yields that $V_{1, \varepsilon}^{n} \approx \varepsilon$ and $V_{2, \varepsilon}^{n} \approx V_{\varepsilon}^{n}, n=1 \rightarrow N$. Of course, as noted in the introduction, we require $\Xi_{M}(\cdot)$ for $\left\{U_{1, \varepsilon}^{n}\right\}_{n=0}^{N}$, as opposed to $\Xi(\cdot)$, for this two-layered problem in order to obtain our discrete entropy bound, see (2.54) below; which is required only in the case $d=2$. In the case $d=1$, one can replace $\Xi_{M}(\cdot)$ by $\Xi(\cdot)$. Finally, as $U_{i, \varepsilon}^{0}>0$, one can ensure that $\Xi_{(M)}\left(U_{i, \varepsilon}^{n-1}\right)$ and $\phi_{j}^{-}\left(U_{i, \varepsilon}^{n-1}\right)$ are well defined for $n \geq 1$; see Theorem 2.4 below. 
Remark 2.2. The restriction of $\sigma$ to the linear case (1.2) is not crucial for the analysis in this paper. However, this choice simplifies our considerations and is also more practical. Different choices of $\sigma$ can be incorporated, see Remark 2.2 in [1] for details.

Below we recall some well-known results concerning $S^{h}$ for any $\kappa \in \mathcal{T}^{h}, \chi, z^{h} \in S^{h}, m \in\{0,1\}, p \in[1, \infty]$, $s \in[2, \infty]$ if $d=1$ and $s \in(2, \infty]$ if $d=2$ :

$$
\begin{array}{rlrl}
|\chi|_{m, r, \kappa} & \leq C h_{\kappa}^{-d\left(\frac{1}{p}-\frac{1}{r}\right)}|\chi|_{m, p, \kappa} & & \text { for any } r \in[p, \infty] ; \\
\lim _{h \rightarrow 0}\left\|\left(I-\pi^{h}\right) \eta\right\|_{1, s} & =0 & & \forall \eta \in W^{1, s}(\Omega) ; \\
\left|\left(I-\pi^{h}\right) \eta\right|_{m, s, \kappa} & \leq C h_{\kappa}^{1-m}|\eta|_{1, s, \kappa} & & \forall \eta \in W^{1, s}(\kappa) ; \\
\int_{\kappa} \chi^{2} \mathrm{~d} x & \leq \int_{\kappa} \pi^{h}\left[\chi^{2}\right] \mathrm{d} x \leq(d+2) \int_{\kappa} \chi^{2} \mathrm{~d} x ; & & \\
\left|\left(\chi, z^{h}\right)-\left(\chi, z^{h}\right)^{h}\right| & \leq\left|\left(I-\pi^{h}\right)\left(\chi z^{h}\right)\right|_{0,1} \leq C h^{1+m}|\chi|_{m, p}\left|z^{h}\right|_{1, p^{\prime}} .
\end{array}
$$

It follows from (2.2) and (2.1) that

$$
\left(Q^{h} \eta\right)\left(p_{j}\right)=m_{j}^{-1}\left(\eta, \chi_{j}\right) \quad \forall j \in J \quad \Longrightarrow \quad\left|Q^{h} \eta\right|_{0, \infty} \leq|\eta|_{0, \infty} \quad \forall \eta \in L^{\infty}(\Omega) .
$$

In addition, it holds for $m \in\{0,1\}$ that

$$
\left|\left(I-Q^{h}\right) \eta\right|_{m, r} \leq C h^{1-m}|\eta|_{1, r} \quad \forall \eta \in W^{1, r}(\Omega) \quad \text { for any } r \in[2, \infty] .
$$

We note that assumption (A) and (1.11) yield that

$$
\int_{\kappa} \nabla z^{h} \cdot \nabla \pi^{h}\left[F_{\varepsilon}^{\prime}\left(z^{h}\right)\right] \mathrm{d} x \geq\left|z^{h}\right|_{1, \kappa}^{2} \quad \forall z^{h} \in S^{h}, \quad \forall \kappa \in \mathcal{T}^{h},
$$

see (2.13) in [1] for details. On setting $Z^{h}:=\left\{z^{h} \in S^{h}:\left(z^{h}, 1\right)^{h}=0\right\}$, it is easily established that

$$
\left|z^{h}\right|_{0, q} \leq C h^{-1}\left\|\mathcal{G} z^{h}\right\|_{1, q} \quad \forall z^{h} \in Z^{h} \quad \text { for any } q \in(1,2] .
$$

We note that the results (2.11) and (2.13) above exploit the fact that we have a quasi-uniform family of partitionings $\left\{\mathcal{T}^{h}\right\}_{h>0}$. Finally, we introduce the "discrete Laplacian" operator $\Delta^{h}: S^{h} \rightarrow S^{h}$ such that

$$
\left(\Delta^{h} z^{h}, \chi\right)^{h}=-\left(\nabla z^{h}, \nabla \chi\right) \quad \forall \chi \in S^{h} .
$$

We introduce for any $\varepsilon \in(0,1)$, on recalling (1.18) and (1.19), the regularized functions $G_{\varepsilon}: \mathbb{R} \rightarrow \mathbb{R}_{\geq 0}$ and $G_{M, \varepsilon}: \mathbb{R} \rightarrow \mathbb{R}_{\geq 0}$ such that

$$
G_{(M,) \varepsilon}(s):= \begin{cases}G_{(M)}(s)+(s-\varepsilon) G_{(M)}^{\prime}(\varepsilon)+\frac{(s-\varepsilon)^{2}}{2} G_{(M)}^{\prime \prime}(\varepsilon) & s \leq \varepsilon \\ G_{(M)}(s) & s \geq \varepsilon\end{cases}
$$

Furthermore, we define $\Xi_{\varepsilon}: S^{h} \rightarrow\left[L^{\infty}(\Omega)\right]^{d \times d}$ and $\Xi_{M, \varepsilon}: S^{h} \rightarrow\left[L^{\infty}(\Omega)\right]^{d \times d}$, for any $\varepsilon \in(0,1)$, such that for all $z^{h} \in S^{h}$ the analogues of $(2.3 \mathrm{a}, \mathrm{b})$ with $G_{(M)}$ replaced by $G_{(M,) \varepsilon}$, respectively, hold. 
Lemma 2.3. Let the assumptions (A) hold. Then for any given $\varepsilon \in(0,1), M \geq 1$ and $\alpha \in(0, \infty)$ the functions $\Lambda_{\varepsilon}, \Xi_{\varepsilon}, \Xi_{M, \varepsilon}: S^{h} \rightarrow\left[L^{\infty}(\Omega)\right]^{d \times d}$ and $\Xi, \Xi_{M}: S_{>0}^{h} \rightarrow\left[L^{\infty}(\Omega)\right]^{d \times d}$ are continuous and satisfy for all $z^{h} \in S^{h}$, $\chi \in S_{>0}^{h}, \xi \in \mathbb{R}^{d}$ and $\kappa \in \mathcal{T}^{h}$

$$
\begin{aligned}
& \varepsilon \xi^{T} \xi \leq\left.\xi^{T} \Lambda_{\varepsilon}\left(z^{h}\right)\right|_{\kappa} \xi \leq \xi^{T} \xi, \quad \varepsilon^{\alpha} \xi^{T} \xi \leq\left.\xi^{T}\left[\Xi_{\varepsilon}\left(z^{h}\right)\right]^{\alpha}\right|_{\kappa} \xi \leq \max _{x \in \bar{\kappa}}\left|\max \left\{\varepsilon, z^{h}(x)\right\}\right|^{\alpha} \xi^{T} \xi, \\
& \varepsilon^{\alpha} \xi^{T} \xi \leq\left.\xi^{T}\left[\Xi_{M, \varepsilon}\left(z^{h}\right)\right]^{\alpha}\right|_{\kappa} \xi \leq M^{\alpha} \xi^{T} \xi, \quad \min _{x \in \bar{\kappa}}[\chi(x)]^{\alpha} \xi^{T} \xi \leq\left.\xi^{T}[\Xi(\chi)]^{\alpha}\right|_{\kappa} \xi \leq \max _{x \in \bar{\kappa}}[\chi(x)]^{\alpha} \xi^{T} \xi, \\
& \min _{x \in \bar{\kappa}}[\min \{\chi(x), M\}]^{\alpha} \xi^{T} \xi \leq\left.\xi^{T}\left[\Xi_{M}(\chi)\right]^{\alpha}\right|_{\kappa} \xi \leq M^{\alpha} \xi^{T} \xi ;
\end{aligned}
$$

and

$$
\begin{gathered}
\max _{x \in \kappa}\left\|\left\{\Lambda_{\varepsilon}\left(z^{h}\right)-\beta_{\varepsilon}^{1}\left(z^{h}\right) \mathcal{I}\right\}(x)\right\| \leq h_{\kappa}\left|\nabla z^{h}\right|_{\kappa} \mid, \\
\max _{x \in \kappa}\left\|\left\{[\Xi(\chi)]^{\alpha}-[\chi]^{\alpha} \mathcal{I}\right\}(x)\right\| \leq \max \{\alpha, 1\}|\chi|_{0, \infty, \kappa}^{\max \{\alpha-1,0\}}\left[h_{\kappa}|\nabla \chi|_{\kappa} \mid\right]^{\min \{\alpha, 1\}}, \\
\max _{x \in \kappa}\left\|\left\{\left[\Xi_{M}(\chi)\right]^{\alpha}-\left[\beta^{M}(\chi)\right]^{\alpha} \mathcal{I}\right\}(x)\right\| \leq \max \{\alpha, 1\}\left|\beta^{M}(\chi)\right|_{0, \infty, \kappa}^{\max \{\alpha-1,0\}}\left[h_{\kappa}|\nabla \chi|_{\kappa} \mid\right]^{\min \{\alpha, 1\}} ;
\end{gathered}
$$

where $\mathcal{I}$ is the $d \times d$ identity matrix.

Proof. For the proof of continuity and (2.16) for $\Lambda_{\varepsilon}$ and $\Xi_{(\varepsilon)}$, see Lemma 2.1 in [2] and Lemma 2.2 in [1]. For the proof of $(2.17 \mathrm{a}, \mathrm{b})$, see Lemma 2.3 in [1]. These proofs are easily adapted to yield continuity and (2.16) for $\Xi_{M(, \varepsilon)}$, and $(2.17 \mathrm{c})$.

As in [1], it is convenient to split $\Phi_{j}$, recall (1.15), into its convex and concave parts. We have for given $a_{j} \in \mathbb{R}_{\geq 0}, \delta_{j} \in \mathbb{R}_{\geq 0}$ and $\nu_{j}>3$ that for all $s \in \mathbb{R}_{>0}$

$$
\Phi_{j}(s)=\Phi_{j}^{+}(s)+\Phi_{j}^{-}(s), \quad \text { where } \quad \Phi_{j}^{+}(s):=\frac{\delta_{j}}{\nu_{j}-1} s^{1-\nu_{j}}, \quad \Phi_{j}^{-}(s):=-\frac{a_{j}}{2} s^{-2}, \quad j=1 \rightarrow 3 .
$$

It holds, on recalling (1.3), that $\phi_{j}^{+} \equiv\left(\Phi_{j}^{+}\right)^{\prime}$ and $\phi_{j}^{-} \equiv\left(\Phi_{j}^{-}\right)^{\prime}$. For future reference, we note as $\delta_{j}>0, j=1,2$, that

$$
\begin{aligned}
\Phi_{j}^{-}(s) & \geq-\frac{a_{j}\left(\nu_{j}-3\right)}{2\left(\nu_{j}-1\right)}\left(\frac{2 a_{j}}{\delta_{j}}\right)^{\frac{2}{\nu_{j}-3}}-\frac{1}{2} \Phi_{j}^{+}(s) \quad \forall s \in \mathbb{R}_{>0}, \quad j=1,2 \\
\Phi_{1}^{-}\left(s_{1}\right)+\Phi_{3}^{-}\left(s_{1}+s_{2}\right) & \geq \Phi_{1}^{-}\left(s_{1}\right)+\Phi_{3}^{-}\left(s_{1}\right) \geq-\frac{\left(a_{1}+a_{3}\right)\left(\nu_{1}-3\right)}{2\left(\nu_{1}-1\right)}\left(\frac{2\left(a_{1}+a_{3}\right)}{\delta_{1}}\right)^{\frac{2}{\nu_{1}-3}}-\frac{1}{2} \Phi_{1}^{+}(s) \quad \forall s_{1}, s_{2} \in \mathbb{R}_{>0} .
\end{aligned}
$$

As $\psi: \mathbb{R}_{>0} \rightarrow \mathbb{R}$, where $\psi(r):=\gamma \phi_{j}^{+}(r)-G_{(M)}^{\prime}(r)+C(\gamma) r$, is monotonically increasing for any $\gamma>0$ with $C(\gamma)$ sufficiently large; we have that for all $\gamma>0$, there exists a constant $C(\gamma)>0$ such that

$$
\left(\nabla \pi^{h}\left[G_{(M)}^{\prime}(\chi)\right], \nabla \chi\right) \leq \gamma\left(\nabla \pi^{h}\left[\phi_{j}^{+}(\chi)\right], \nabla \chi\right)+C(\gamma)|\chi|_{1}^{2} \quad \forall \chi \in S_{>0}^{h}, \quad j=1,2 .
$$

In addition, if $\nu_{j} \geq 7, j=1,2$, one can deduce that

$$
\left[\phi_{j}^{-}\left(s_{2}\right)-\phi_{j}^{-}\left(s_{1}\right)\right]^{2} \leq C_{1}\left(\delta_{j}, \nu_{j}, a_{j}\right)\left[\phi_{j}^{+}\left(s_{2}\right)-\phi_{j}^{+}\left(s_{1}\right)\right]\left(s_{2}-s_{1}\right)+C_{2}\left(a_{j}\right)\left(s_{2}-s_{1}\right)^{2} \quad \forall s_{2}, s_{1}>0, \quad j=1,2 ;
$$


see e.g. Lemma 4.4 in [6], and hence that

$$
\left|\nabla \pi^{h}\left[\phi_{j}^{-}(\chi)\right]\right|_{0}^{2} \leq C_{1}\left(\delta_{j}, \nu_{j}, a_{j}\right)\left(\nabla \pi^{h}\left[\phi_{j}^{+}(\chi)\right], \nabla \chi\right)+C_{2}\left(a_{j}\right)|\chi|_{1}^{2} \quad \forall \chi \in S_{>0}^{h}, \quad j=1,2 .
$$

We note also that for all $s_{i}>0$

$$
\left[\phi_{3}^{-}\left(s_{1}+s_{2}\right)-\phi_{3}^{-}\left(s_{3}+s_{4}\right)\right]^{2} \leq 2\left[\phi_{3}^{-}\left(s_{1}\right)-\phi_{3}^{-}\left(s_{3}\right)\right]^{2}+2\left[\phi_{3}^{-}\left(s_{2}\right)-\phi_{3}^{-}\left(s_{4}\right)\right]^{2} .
$$

Hence, similarly to (2.22), we have that

$$
\left|\nabla \pi^{h}\left[\phi_{3}^{-}\left(\chi_{1}+\chi_{2}\right)\right]\right|_{0}^{2} \leq \sum_{j=1}^{2}\left[C_{1}\left(\delta_{j}, \nu_{j}, a_{3}\right)\left(\nabla \pi^{h}\left[\phi_{j}^{+}\left(\chi_{j}\right)\right], \nabla \chi_{j}\right)+C_{2}\left(a_{3}\right)\left|\chi_{j}\right|_{1}^{2}\right] \quad \forall \chi_{1}, \chi_{2} \in S_{>0}^{h}
$$

Similarly to the proof of (2.22), one can also show that

$$
\left|\Xi(\chi) \nabla \pi^{h}\left[G^{\prime}(\chi)\right]\right|_{0}^{2} \leq C_{1}\left(\delta_{2}, \nu_{2}\right)\left(\nabla \pi^{h}\left[\phi_{2}^{+}(\chi)\right], \nabla \chi\right)+C_{2}\left(\delta_{2}, \nu_{2}\right)|\chi|_{1}^{2} \quad \forall \chi \in S_{>0}^{h}
$$

To prove existence of a solution to $\left(\mathbf{P}_{\varepsilon}^{h, \tau}\right)$ we need to go through a regularization procedure, which is similar to that used in Theorem 2.1 in [1]. For this purpose we introduce for any $\zeta \in \mathbb{R}_{>0}$, on recalling (2.18), the $C^{2,1}$ convex (concave) functions for $j=1 \rightarrow 3, \Phi_{j}^{ \pm}: \mathbb{R} \rightarrow \mathbb{R}_{\geq 0}$ such that

$$
\Phi_{j, \zeta}^{ \pm}(s):= \begin{cases}\Phi_{j}^{ \pm}(\zeta)+(s-\zeta) \phi_{j}^{ \pm}(\zeta)+\frac{(s-\zeta)^{2}}{2}\left(\phi_{j}^{ \pm}\right)^{\prime}(\zeta) & s \leq \zeta \\ \Phi_{j}^{ \pm}(s) & \zeta \leq s\end{cases}
$$

We set $\phi_{j, \zeta}^{ \pm}:=\left(\Phi_{j, \zeta}^{ \pm}\right)^{\prime}, \Phi_{j, \zeta}:=\Phi_{j, \zeta}^{+}+\Phi_{j, \zeta}^{-}$and note that $\Phi_{j}^{+}(s) \geq \Phi_{j, \zeta}^{+}(s) \geq 0 \geq \Phi_{j, \zeta}^{-}(s) \geq \Phi_{j}^{-}(s)$ for all $s \in \mathbb{R}_{>0}$. Furthermore, $\Phi_{j}, j=1,2$ is strictly monotonically decreasing on $\left(0,\left[\frac{\delta_{j}}{a_{j}}\right]^{\frac{1}{\left(\nu_{j}-3\right)}}\right),(0, \infty)$ if $a_{j}=0$, and $\Phi_{j}^{-1}$ is uniquely defined on $\mathbb{R}_{\geq 0}$. Finally, we recall the well-known identity

$$
2 r(r-s)=\left(r^{2}-s^{2}\right)+(r-s)^{2} .
$$

Theorem 2.4. Let $\phi_{j}(\cdot)$ satisfy (1.3) with $\delta_{j}>0, j=1,2$. Let the assumptions (A) hold and $\left\{U_{i, \varepsilon}^{n-1}, V_{i, \varepsilon}^{n-1}\right\}$ $\in S_{>0}^{h} \times S^{h}, i=1,2$. Then for all $\varepsilon \in(0,1)$ and for all $h, \tau>0$ there exists a solution $\left\{U_{i, \varepsilon}^{n}, W_{i, \varepsilon}^{n}, V_{i, \varepsilon}^{n}\right\} \in$ $\left[S_{>0}^{h}\right] \times\left[S^{h}\right]^{2}, i=1,2$, to the $n$-th step of $\left(\mathrm{P}_{\varepsilon}^{h, \tau}\right)$ with $f U_{i, \varepsilon}^{n}=f U_{i, \varepsilon}^{n-1}$ and $f V_{i, \varepsilon}^{n}=f V_{i, \varepsilon}^{n-1}, i=1,2$.

Proof. As noted in Remark 2.1, the system $\left(\mathbf{P}_{\varepsilon}^{h, \tau}\right)$ decouples. We introduce the following regularized version of $(2.4 \mathrm{a}-\mathrm{d})$ of $\left(\mathbf{P}_{\varepsilon}^{h, \tau}\right)$ at time level $t_{n}$ for any fixed $\mathcal{T}^{h}, \varepsilon \in(0,1)$ and $\zeta>0$ : Find $\left\{U_{i, \varepsilon, \zeta}^{n}, W_{i, \varepsilon, \zeta}^{n}\right\}_{i=1}^{2} \in\left[S^{h}\right]^{4}$ 
such that for all $\chi \in S^{h}$

$$
\begin{gathered}
\mu\left(\frac{U_{1, \varepsilon, \zeta}^{n}-U_{1, \varepsilon}^{n-1}}{\tau}, \chi\right)^{h}+\frac{1}{3}\left(\left[\Xi_{M, \zeta}\left(U_{1, \varepsilon, \zeta}^{n}\right)\right]^{3} \nabla W_{1, \varepsilon, \zeta}^{n}, \nabla \chi\right)+\frac{1}{2}\left(\left[\Xi_{M, \zeta}\left(U_{1, \varepsilon, \zeta}^{n}\right)\right]^{2} \Xi_{\zeta}\left(U_{2, \varepsilon, \zeta}^{n}\right) \nabla W_{2, \varepsilon, \zeta}^{n}, \nabla \chi\right) \\
=-\frac{1}{2}\left(\left[\Xi_{M, \zeta}\left(U_{1, \varepsilon, \zeta}^{n}\right)\right]^{\frac{3}{2}}\left[\Xi_{M}\left(U_{1, \varepsilon}^{n-1}\right)\right]^{\frac{1}{2}} \nabla\left[V_{1, \varepsilon}^{n-1}+V_{2, \varepsilon}^{n-1}\right], \nabla \chi\right) \\
\mu\left(\frac{U_{2, \varepsilon, \zeta}^{n}-U_{2, \varepsilon}^{n-1}}{\tau}, \chi\right)^{h}+\frac{\mu}{3}\left(\left[\Xi_{\zeta}\left(U_{2, \varepsilon, \zeta}^{n}\right)\right]^{3} \nabla W_{2, \varepsilon, \zeta}^{n}, \nabla \chi\right)+\left(\Xi_{M, \zeta}\left(U_{1, \varepsilon, \zeta}^{n}\right)\left[\Xi_{\zeta}\left(U_{2, \varepsilon, \zeta}^{n}\right)\right]^{2} \nabla W_{2, \varepsilon, \zeta}^{n}, \nabla \chi\right) \\
+\frac{1}{2}\left(\left[\Xi_{M, \zeta}\left(U_{1, \varepsilon, \zeta}^{n}\right)\right]^{2} \Xi_{\zeta}\left(U_{2, \varepsilon, \zeta}^{n}\right) \nabla W_{1, \varepsilon, \zeta}^{n}, \nabla \chi\right) \\
=-\frac{\mu}{2}\left(\left[\Xi_{\zeta}\left(U_{2, \varepsilon, \zeta}^{n}\right)\right]^{\frac{3}{2}}\left[\Xi_{2, \varepsilon}\left(U_{2, \varepsilon}^{n-1}\right)\right]^{\frac{1}{2}} \nabla V_{2, \varepsilon}^{n-1}, \nabla \chi\right) \\
\quad-\left(\left[\Xi_{M, \zeta}\left(U_{1, \varepsilon, \zeta}^{n}\right)\right]^{\frac{1}{2}}\left[\Xi_{M}\left(U_{1, \varepsilon}^{n-1}\right)\right]^{\frac{1}{2}} \Xi_{\zeta}\left(U_{2, \varepsilon, \zeta}^{n}\right) \nabla\left[V_{1, \varepsilon}^{n-1}+V_{2, \varepsilon}^{n-1}\right], \nabla \chi\right) \\
c_{1}\left(\nabla U_{1, \varepsilon, \zeta}^{n}, \nabla \chi\right)+\left(\phi_{1, \zeta}^{+}\left(U_{1, \varepsilon, \zeta}^{n}\right)+\phi_{1, \zeta}^{-}\left(U_{1, \varepsilon}^{n-1}\right), \chi\right)^{h}-\left(\phi_{2, \zeta}^{+}\left(U_{2, \varepsilon, \zeta}^{n}\right)+\phi_{2, \zeta}^{-}\left(U_{2, \varepsilon}^{n-1}\right), \chi\right)^{h} \\
=\left(W_{1, \varepsilon, \zeta}^{n}-W_{2, \varepsilon, \zeta}^{n}, \chi\right)^{h} \\
c_{2}\left(\nabla\left[U_{1, \varepsilon, \zeta}^{n}+U_{2, \varepsilon, \zeta}^{n}\right], \nabla \chi\right)+\left(\phi_{2, \zeta}^{+}\left(U_{2, \varepsilon, \zeta}^{n}\right)+\phi_{2, \zeta}^{-}\left(U_{2, \varepsilon}^{n-1}\right), \chi\right)^{h} \\
+\left(\phi_{3, \zeta}^{+}\left(U_{1, \varepsilon, \zeta}^{n}+U_{2, \varepsilon, \zeta}^{n}\right)+\phi_{3, \zeta}^{-}\left(U_{1, \varepsilon}^{n-1}+U_{2, \varepsilon}^{n-1}\right), \chi\right)^{h}=\left(W_{2, \varepsilon, \zeta}^{n}, \chi\right)^{h} .
\end{gathered}
$$

To prove existence of a solution to $(2.28 \mathrm{a}-\mathrm{d})$ we introduce for $i=1,2, m_{i}=f U_{i, \varepsilon}^{n-1}$ and $D_{i, \zeta}: Z^{h} \times Z^{h} \rightarrow Z^{h}$ such that for any $\left(\bar{U}_{1}, \bar{U}_{2}\right) \in Z^{h} \times Z^{h}$

$$
\begin{aligned}
& \left(D_{1, \zeta}\left(\bar{U}_{1}, \bar{U}_{2}\right), \chi\right)^{h}:=\mu\left(\bar{U}_{1}-(I-f) U_{1, \varepsilon}^{n-1}, \chi\right)^{h}+\frac{\tau}{2}\left(\left[\Xi_{M, \zeta}\left(\bar{U}_{1}+m_{1}\right)\right]^{2} \Xi_{\zeta}\left(\bar{U}_{2}+m_{2}\right) \nabla \bar{W}_{2}, \nabla \chi\right) \\
& \quad+\frac{\tau}{2}\left(\left[\Xi_{M, \zeta}\left(\bar{U}_{1}+m_{1}\right)\right]^{\frac{3}{2}}\left[\Xi_{M}\left(U_{1, \varepsilon}^{n-1}\right)\right]^{\frac{1}{2}} \nabla\left[\sum_{i=1}^{2} V_{i, \varepsilon}^{n-1}\right], \nabla \chi\right)+\frac{\tau}{3}\left(\left[\Xi_{M, \zeta}\left(\bar{U}_{1}+m_{1}\right)\right]^{3} \nabla \bar{W}_{1}, \nabla \chi\right), \\
& \left(D_{2, \zeta}\left(\bar{U}_{1}, \bar{U}_{2}\right), \chi\right)^{h}:=\mu\left(\bar{U}_{2}-(I-f) U_{2, \varepsilon}^{n-1}, \chi\right)^{h}+\tau\left(\Xi_{M, \zeta}\left(\bar{U}_{1}+m_{1}\right)\left[\Xi_{\zeta}\left(\bar{U}_{2}+m_{2}\right)\right]^{2} \nabla \bar{W}_{2}, \nabla \chi\right) \\
& \quad+\frac{\tau}{2}\left(\left[\Xi_{M, \zeta}\left(\bar{U}_{1}+m_{1}\right)\right]^{2} \Xi_{\zeta}\left(\bar{U}_{2}+m_{2}\right) \nabla \bar{W}_{1}, \nabla \chi\right)+\frac{\mu \tau}{3}\left(\left[\Xi_{\zeta}\left(\bar{U}_{2}+m_{2}\right)\right]^{3} \nabla \bar{W}_{2}, \nabla \chi\right) \\
& \quad+\tau\left(\left[\Xi_{M, \zeta}\left(\bar{U}_{1}+m_{1}\right)\right]^{\frac{1}{2}} \Xi_{\zeta}\left(\bar{U}_{2}+m_{2}\right)\left[\Xi_{M}\left(U_{1, \varepsilon}^{n-1}\right)\right]^{\frac{1}{2}} \nabla\left[V_{1, \varepsilon}^{n-1}+V_{2, \varepsilon}^{n-1}\right], \nabla \chi\right) \\
& \quad+\frac{\mu \tau}{2}\left(\left[\Xi_{\zeta}\left(\bar{U}_{2}+m_{2}\right)\right]^{\frac{3}{2}}\left[\Xi\left(U_{2, \varepsilon}^{n-1}\right)\right]^{\frac{1}{2}} \nabla V_{2, \varepsilon}^{n-1}, \nabla \chi\right)
\end{aligned}
$$


where

$$
\begin{aligned}
\bar{W}_{1}=-\left(c_{1}+c_{2}\right) \Delta^{h} \bar{U}_{1}-c_{2} \Delta^{h} \bar{U}_{2}+ & \pi^{h}\left[\phi_{1, \zeta}^{+}\left(\bar{U}_{1}+m_{1}\right)+\phi_{1, \zeta}^{-}\left(U_{1, \varepsilon}^{n-1}\right)\right] \\
& +\pi^{h}\left[\phi_{3, \zeta}^{+}\left(\bar{U}_{1}+\bar{U}_{2}+m_{1}+m_{2}\right)+\phi_{3, \zeta}^{-}\left(U_{1, \varepsilon}^{n-1}+U_{2, \varepsilon}^{n-1}\right)\right], \\
\bar{W}_{2}=-c_{2} \Delta^{h}\left(\bar{U}_{1}+\bar{U}_{2}\right)+\pi^{h}\left[\phi _ { 2 , \zeta } ^ { + } \left(\bar{U}_{2}\right.\right. & \left.\left.+m_{2}\right)+\phi_{2, \zeta}^{-}\left(U_{2, \varepsilon}^{n-1}\right)\right] \\
& +\pi^{h}\left[\phi_{3, \zeta}^{+}\left(\bar{U}_{1}+\bar{U}_{2}+m_{1}+m_{2}\right)+\phi_{3, \zeta}^{-}\left(U_{1, \varepsilon}^{n-1}+U_{2, \varepsilon}^{n-1}\right)\right] .
\end{aligned}
$$

Solving the regularized problem $(2.28 \mathrm{a}-\mathrm{d})$ is equivalent to finding $\left\{\bar{U}_{1}, \bar{U}_{2}\right\}$ such that for $i=1,2, D_{i, \zeta}\left(\bar{U}_{1}, \bar{U}_{2}\right)=$ 0 , since given such $\bar{U}_{1}$ and $\bar{U}_{2}$ then $\left\{U_{i, \varepsilon, \zeta}^{n}, W_{i, \varepsilon, \zeta}^{n}\right\}_{i=1}^{2}=\left\{\bar{U}_{i}+m_{i}, \bar{W}_{i}\right\}_{i=1}^{2}$ solves (2.28a-d).

Assume that for a given $R>0$ there does not exist $\left\{\bar{U}_{1}, \bar{U}_{2}\right\} \in Z_{R}^{h}:=\left\{\left\{z_{1}^{h}, z_{2}^{h}\right\} \in\left[Z^{h}\right]^{2}:\left|z_{1}^{h}\right|_{1}^{2}+\left|z_{2}^{h}\right|_{1}^{2} \leq R^{2}\right\}$ with $D_{i, \zeta}\left(\bar{U}_{1}, \bar{U}_{2}\right)=0$ for $i=1,2$. For any fixed $\zeta>0$ it follows from $(2.29 \mathrm{a}, \mathrm{b}),(2.30 \mathrm{a}, \mathrm{b}),(2.14),(2.26)$ and Lemma 2.3 that $D_{i, \zeta}$ is continuous on $Z_{R}^{h}$. Hence, we can define the continuous function $B_{\zeta}: Z_{R}^{h} \rightarrow Z_{R}^{h}$, where $B_{\zeta}\left(\bar{U}_{1}, \bar{U}_{2}\right)=-\frac{\left\{R D_{1, \zeta}\left(\bar{U}_{1}, \bar{U}_{2}\right), R D_{2, \zeta}\left(\bar{U}_{1}, \bar{U}_{2}\right)\right\}}{\left[\left|D_{1, \zeta}\left(\bar{U}_{1}, \bar{U}_{2}\right)\right|_{1}^{2}+\left|D_{2, \zeta}\left(\bar{U}_{1}, \bar{U}_{2}\right)\right|_{1}^{2}\right]^{\frac{1}{2}}}$. As $Z_{R}^{h}$ is a convex and compact subset of the finite dimensional space $\left[S^{h}\right]^{2}$, the Brouwer fixed point theorem (see e.g. [9], Thm. 9.36, p. 357) asserts that there exists $\left\{\bar{U}_{1}^{R}, \bar{U}_{2}^{R}\right\} \in$ $Z_{R}^{h}$ such that $B_{\zeta}\left(\bar{U}_{1}^{R}, \bar{U}_{2}^{R}\right)=\left\{\bar{U}_{1}^{R}, \bar{U}_{2}^{R}\right\}$. Moreover, $\left|\bar{U}_{1}^{R}\right|_{1}^{2}+\left|\bar{U}_{2}^{R}\right|_{1}^{2}=R^{2}$. We will now prove a contradiction for $R$ sufficiently large. Let $\bar{W}_{1}^{R}$ and $\bar{W}_{2}^{R}$ be defined by $(2.30 \mathrm{a}, \mathrm{b})$ with $\bar{U}_{i}$ replaced by $\bar{U}_{i}^{R}, i=1,2$. Then $(2.14)$, the monotonicity of $\phi_{j, \zeta}^{+},(1.16),(2.8)$ and (1.25) yield for $\gamma>0$ sufficiently small that

$$
\begin{aligned}
\sum_{i=1}^{2}\left(\bar{U}_{i}^{R}, \bar{W}_{i}^{R}\right)^{h}= & c_{1}\left|\bar{U}_{1}^{R}\right|_{1}^{2}+c_{2}\left|\bar{U}_{1}^{R}+\bar{U}_{2}^{R}\right|_{1}^{2}+\sum_{i=1}^{2}\left(\bar{U}_{i}^{R}, \phi_{i, \zeta}^{+}\left(\bar{U}_{i}^{R}+m_{i}\right)+\phi_{i, \zeta}^{-}\left(U_{i, \varepsilon}^{n-1}\right)\right)^{h} \\
& \left.\quad+\left(\bar{U}_{1}^{R}+\bar{U}_{2}^{R}, \phi_{3, \zeta}^{+} \bar{U}_{1}^{R}+m_{1}+\bar{U}_{2}^{R}+m_{2}\right)+\phi_{3, \zeta}^{-}\left(U_{1, \varepsilon}^{n-1}+U_{2, \varepsilon}^{n-1}\right)\right)^{h} \\
\geq & c_{1}\left|\bar{U}_{1}^{R}\right|_{1}^{2}+c_{2}\left|\bar{U}_{1}^{R}+\bar{U}_{2}^{R}\right|_{1}^{2}-\gamma\left[\left|\bar{U}_{1}^{R}\right|_{h}^{2}+\left|\bar{U}_{1}^{R}+\bar{U}_{2}^{R}\right|_{h}^{2}\right]-C\left(\gamma,\left\{U_{i, \varepsilon}^{n-1}\right\}_{i=1}^{2}\right) \\
\geq & \frac{c_{1}}{2}\left|\bar{U}_{1}^{R}\right|_{1}^{2}+\frac{c_{2}}{2}\left|\bar{U}_{1}^{R}+\bar{U}_{2}^{R}\right|_{1}^{2}-C\left(\gamma,\left\{U_{i, \varepsilon}^{n-1}\right\}_{i=1}^{2}\right) \\
\geq & \frac{c_{\min }}{8}\left[\left|\bar{U}_{1}^{R}\right|_{1}^{2}+\left|\bar{U}^{R}\right|_{1}^{2}\right]-C\left(\gamma,\left\{U_{i, \varepsilon}^{n-1}\right\}_{i=1}^{2}\right)=\frac{c_{\min } R^{2}}{8}-C\left(\gamma,\left\{U_{i, \varepsilon}^{n-1}\right\}_{i=1}^{2}\right),
\end{aligned}
$$

where $c_{\min }:=\min \left\{c_{1}, c_{2}\right\}$. Similarly, on noting in addition $(2.27)$, we have for $\zeta \in\left(0, \zeta_{1}\right]$, where $\zeta_{1}:=$ $\min \left\{\min _{x \in \bar{\Omega}} U_{1, \varepsilon}^{n-1}(x), \min _{x \in \bar{\Omega}} U_{2, \varepsilon}^{n-1}(x)\right\}$, that for $\gamma>0$ sufficiently small

$$
\begin{aligned}
\sum_{i=1}^{2}\left(\bar{U}_{i}^{R}+\right. & \left.m_{i}-U_{i, \varepsilon}^{n-1}, \bar{W}_{i}^{R}\right)^{h} \\
\geq & \frac{c_{1}}{2}\left|\bar{U}_{1}^{R}\right|_{1}^{2}+\frac{c_{2}}{2}\left|\bar{U}_{1}^{R}+\bar{U}_{2}^{R}\right|_{1}^{2}+\sum_{i=1}^{2}\left(\bar{U}_{i}^{R}+m_{i}-U_{i, \varepsilon}^{n-1}, \phi_{i, \zeta}^{+}\left(\bar{U}_{i}^{R}+m_{i}\right)+\phi_{i, \zeta}^{-}\left(U_{i, \varepsilon}^{n-1}\right)\right)^{h} \\
& +\left(\sum_{i=1}^{2}\left[\bar{U}_{i}^{R}+m_{i}-U_{i, \varepsilon}^{n-1}\right], \phi_{3, \zeta}^{+}\left(\bar{U}_{1}^{R}+m_{1}+\bar{U}_{2}^{R}+m_{2}\right)+\phi_{3, \zeta}^{-}\left(U_{1, \varepsilon}^{n-1}+U_{2, \varepsilon}^{n-1}\right)\right)^{h}-C\left(\left\{U_{i, \varepsilon}^{n-1}\right\}_{i=1}^{2}\right) \\
\geq & \frac{c_{1}}{2}\left|\bar{U}_{1}^{R}\right|_{1}^{2}+\frac{c_{2}}{2}\left|\bar{U}_{1}^{R}+\bar{U}_{2}^{R}\right|_{1}^{2}+\sum_{i=1}^{2}\left(\bar{U}_{i}^{R}+m_{i}-U_{i, \varepsilon}^{n-1}, \phi_{i, \zeta}\left(U_{i, \varepsilon}^{n-1}\right)\right)^{h} \\
& +\left(\sum_{i=1}^{2}\left[\bar{U}_{i}^{R}+m_{i}-U_{i, \varepsilon}^{n-1}\right], \phi_{3, \zeta}\left(U_{1, \varepsilon}^{n-1}+U_{2, \varepsilon}^{n-1}\right)\right)^{h}-C\left(\left\{U_{i, \varepsilon}^{n-1}\right\}_{i=1}^{2}\right) \\
\geq & \frac{c_{\min } R^{2}}{16}-C\left(\gamma,\left\{U_{i, \varepsilon}^{n-1}\right\}_{i=1}^{2}\right) .
\end{aligned}
$$


We obtain from $(2.29 \mathrm{a}, \mathrm{b}),(1.16)$ and $(2.32)$ for all $\zeta \in\left(0, \zeta_{1}\right]$ that for $\gamma>0$ sufficiently small

$$
\begin{aligned}
\sum_{i=1}^{2}( & \left.D_{i, \zeta}\left(\bar{U}_{1}^{R}, \bar{U}_{2}^{R}\right), \bar{W}_{i}^{R}\right)^{h} \\
= & \mu\left(\bar{U}_{1}^{R}+m_{1}-U_{1, \varepsilon}^{n-1}, \bar{W}_{1}^{R}\right)^{h}+\mu\left(\bar{U}_{2}^{R}+m_{2}-U_{2, \varepsilon}^{n-1}, \bar{W}_{2}^{R}\right)^{h}+\frac{\tau}{3}\left(\left[\Xi_{M, \zeta}\left(\bar{U}_{1}^{R}+m_{1}\right)\right]^{3} \nabla \bar{W}_{1}^{R}, \nabla \bar{W}_{1}^{R}\right) \\
& +\frac{\mu \tau}{3}\left(\left[\Xi_{\zeta}\left(\bar{U}_{2}^{R}+m_{2}\right)\right]^{3} \nabla \bar{W}_{2}^{R}, \nabla \bar{W}_{2}^{R}\right)+\tau\left(\Xi_{M, \zeta}\left(\bar{U}_{1}^{R}+m_{1}\right)\left[\Xi_{\zeta}\left(\bar{U}_{2}^{R}+m_{2}\right)\right]^{2} \nabla \bar{W}_{2}^{R}, \nabla \bar{W}_{2}^{R}\right) \\
& +\tau\left(\left[\Xi_{M, \zeta}\left(\bar{U}_{1}^{R}+m_{1}\right)\right]^{2} \Xi_{\zeta}\left(\bar{U}_{2}^{R}+m_{2}\right) \nabla \bar{W}_{1}^{R}, \nabla \bar{W}_{2}^{R}\right)+\frac{\mu \tau}{2}\left(\left[\Xi_{\zeta}\left(\bar{U}_{2}^{R}+m_{2}\right)\right]^{\frac{3}{2}}\left[\Xi\left(U_{2, \varepsilon}^{n-1}\right)\right]^{\frac{1}{2}} \nabla V_{2, \varepsilon}^{n-1}, \nabla \bar{W}_{2}^{R}\right) \\
& +\frac{\tau}{2}\left(\left[\Xi_{M, \zeta}\left(\bar{U}_{1}^{R}+m_{1}\right)\right]^{\frac{3}{2}}\left[\Xi_{M}\left(U_{1, \varepsilon}^{n-1}\right)\right]^{\frac{1}{2}} \nabla\left[V_{1, \varepsilon}^{n-1}+V_{2, \varepsilon}^{n-1}\right], \nabla \bar{W}_{1}^{R}\right) \\
& +\tau\left(\left[\Xi_{M, \zeta}\left(\bar{U}_{1}^{R}+m_{1}\right)\right]^{\frac{1}{2}} \Xi_{\zeta}\left(\bar{U}_{2}^{R}+m_{2}\right)\left[\Xi_{M}\left(U_{1, \varepsilon}^{n-1}\right)\right]^{\frac{1}{2}} \nabla\left[V_{1, \varepsilon}^{n-1}+V_{2, \varepsilon}^{n-1}\right], \nabla \bar{W}_{2}^{R}\right) \\
\geq & \mu\left(\bar{U}_{1}^{R}+m_{1}-U_{1, \varepsilon}^{n-1}, \bar{W}_{1}^{R}\right)^{h}+\mu\left(\bar{U}_{2}^{R}+m_{2}-U_{2, \varepsilon}^{n-1}, \bar{W}_{2}^{R}\right)^{h}-C\left(\tau, \gamma,\left\{U_{i, \varepsilon}^{n-1}, V_{i, \varepsilon}^{n-1}\right\}_{i=1}^{2}\right) \\
\geq & \frac{\mu c_{\min } R^{2}}{16}-C\left(\tau, h, \gamma, \mu,\left\{U_{i, \varepsilon}^{n-1}, V_{i, \varepsilon}^{n-1}\right\}_{i=1}^{2}\right) .
\end{aligned}
$$

Hence $(2.31)$ and $(2.33)$ yield for any $\zeta \in\left(0, \zeta_{1}\right]$ that

$$
\sum_{i=1}^{2}\left(\bar{U}_{i}^{R}, \bar{W}_{i}^{R}\right)^{h}>0 \quad \text { and } \quad \sum_{i=1}^{2}\left(D_{i, \zeta}\left(\bar{U}_{1}^{R}, \bar{U}_{2}^{R}\right), \bar{W}_{i}^{R}\right)^{h}>0 \quad \text { for } R \text { sufficiently large. }
$$

However, $\left\{\bar{U}_{1}, \bar{U}_{2}\right\}$ is a fixed point of $B_{\zeta}(\cdot, \cdot)$ and so

$$
\sum_{i=1}^{2}\left(\bar{U}_{1}^{R}, \bar{W}_{1}^{R}\right)^{h}=-\frac{R \sum_{i=1}^{2}\left(D_{i, \zeta}\left(\bar{U}_{1}^{R}, \bar{U}_{2}^{R}\right), \bar{W}_{i}^{R}\right)^{h}}{\left(\left|D_{1, \zeta}\left(\bar{U}_{1}^{R}\right)\right|_{1}^{2}+\left|D_{2, \zeta}\left(\bar{U}_{2}^{R}\right)\right|_{1}^{2}\right)^{\frac{1}{2}}}
$$

which clearly contradicts (2.34). Therefore, we have existence of a solution $\left\{U_{i, \varepsilon, \zeta}^{n}, W_{i, \varepsilon, \zeta}^{n}\right\}_{i=1}^{2}$ to $(2.28 \mathrm{a}-\mathrm{d})$ for all $\zeta \in\left(0, \zeta_{1}\right]$.

We will now show that for $\zeta$ sufficiently small, $\left\{U_{i, \varepsilon, \zeta}^{n}, W_{i, \varepsilon, \zeta}^{n}\right\}_{i=1}^{2}$ solves $(2.4 \mathrm{a}-\mathrm{d})$. Choosing $\chi=W_{1, \varepsilon, \zeta}^{n}$ in (2.28a), $\chi=W_{2, \varepsilon, \zeta}^{n}$ in (2.28b), and summing yields that

$$
\begin{gathered}
{\left[\mu \sum_{i=1}^{2}\left(U_{i, \varepsilon, \zeta}^{n}-U_{i, \varepsilon}^{n-1}, W_{i, \varepsilon, \zeta}^{n}\right)^{h}\right]+\frac{\tau}{3}\left(\left[\Xi_{M, \zeta}\left(U_{1, \varepsilon, \zeta}^{n}\right)\right]^{3} \nabla W_{1, \varepsilon, \zeta}^{n}, \nabla W_{1, \varepsilon, \zeta}^{n}\right)} \\
+\tau \frac{\mu}{3}\left(\left[\Xi_{\zeta}\left(U_{2, \varepsilon, \zeta}^{n}\right)\right]^{3} \nabla W_{2, \varepsilon, \zeta}^{n}, \nabla W_{2, \varepsilon, \zeta}^{n}\right)+\tau\left(\Xi_{M, \zeta}\left(U_{1, \varepsilon, \zeta}^{n}\right)\left[\Xi_{\zeta}\left(U_{2, \varepsilon, \zeta}^{n}\right)\right]^{2} \nabla W_{2, \varepsilon, \zeta}^{n}, \nabla W_{2, \varepsilon, \zeta}^{n}\right) \\
+\tau\left(\left[\Xi_{M, \zeta}\left(U_{1, \varepsilon, \zeta}^{n}\right)\right]^{2} \Xi_{\zeta}\left(U_{2, \varepsilon, \zeta}^{n}\right) \nabla W_{2, \varepsilon, \zeta}^{n}, \nabla W_{1, \varepsilon, \zeta}^{n}\right) \\
=-\frac{\tau}{2}\left(\left[\Xi_{M, \zeta}\left(U_{1, \varepsilon, \zeta}^{n}\right)\right]^{\frac{3}{2}}\left[\Xi_{M}\left(U_{1, \varepsilon}^{n-1}\right)\right]^{\frac{1}{2}} \nabla\left[V_{1, \varepsilon}^{n-1}+V_{2, \varepsilon}^{n-1}\right], \nabla W_{1, \varepsilon, \zeta}^{n}\right) \\
-\tau\left(\left[\Xi_{M, \zeta}\left(U_{1, \varepsilon, \zeta}^{n}\right)\right]^{\frac{1}{2}}\left[\Xi_{M}\left(U_{1, \varepsilon}^{n-1}\right)\right]^{\frac{1}{2}} \Xi_{\zeta}\left(U_{2, \varepsilon, \zeta}^{n}\right) \nabla\left[V_{1, \varepsilon}^{n-1}+V_{2, \varepsilon}^{n-1}\right], \nabla W_{2, \varepsilon, \zeta}^{n}\right) \\
-\tau \frac{\mu}{2}\left(\left[\Xi_{\zeta}\left(U_{2, \varepsilon, \zeta}^{n}\right)\right]^{\frac{3}{2}}\left[\Xi\left(U_{2, \varepsilon}^{n-1}\right)\right]^{\frac{1}{2}} \nabla V_{2, \varepsilon}^{n-1}, \nabla W_{2, \varepsilon, \zeta}^{n}\right) .
\end{gathered}
$$


Choosing $\chi=U_{1, \varepsilon, \zeta}^{n}-U_{1, \varepsilon}^{n-1}$ in $(2.28 \mathrm{c}), \chi=\sum_{i=1}^{2}\left(U_{i, \varepsilon, \zeta}^{n}-U_{i, \varepsilon}^{n-1}\right)$ in $(2.28 \mathrm{~d})$, and summing yields that

$$
\begin{gathered}
c_{1}\left(\nabla U_{1, \varepsilon, \zeta}^{n}, \nabla\left[U_{1, \varepsilon, \zeta}^{n}-U_{1, \varepsilon}^{n-1}\right]\right)+c_{2}\left(\nabla\left[\sum_{i=1}^{2} U_{i, \varepsilon, \zeta}^{n}\right], \nabla\left[\sum_{i=1}^{2} U_{i, \varepsilon, \zeta}^{n}-U_{i, \varepsilon}^{n-1}\right]\right) \\
+\sum_{i=1}^{2}\left(\phi_{i, \zeta}^{+}\left(U_{i, \varepsilon, \zeta}^{n}\right)+\phi_{1, \zeta}^{-}\left(U_{i, \varepsilon}^{n-1}\right), U_{i, \varepsilon, \zeta}^{n}-U_{i, \varepsilon}^{n-1}\right)^{h}+\left(\phi_{3, \zeta}^{+}\left(\sum_{i=1}^{2} U_{i, \varepsilon, \zeta}^{n}\right)+\phi_{3, \zeta}^{-}\left(\sum_{i=1}^{2} U_{i, \varepsilon}^{n-1}\right), \sum_{i=1}^{2}\left[U_{i, \varepsilon, \zeta}^{n}-U_{i, \varepsilon}^{n-1}\right]\right)^{h} \\
=\sum_{i=1}^{2}\left(W_{i, \varepsilon, \zeta}^{n}, U_{i, \varepsilon, \zeta}^{n}-U_{i, \varepsilon}^{n-1}\right)^{h} .
\end{gathered}
$$

On noting (2.27), the convexity of $\Phi_{j, \zeta}^{+}$and the concavity of $\Phi_{j, \zeta}^{-}, j=1 \rightarrow 3$, it follows from (2.36) that

$$
\begin{aligned}
& \frac{c_{1}}{2}\left[\left|U_{1, \varepsilon, \zeta}^{n}\right|_{1}^{2}+\left|U_{1, \varepsilon, \zeta}^{n}-U_{1, \varepsilon}^{n-1}\right|_{1}^{2}\right]+\frac{c_{2}}{2}\left[\left|\sum_{i=1}^{2} U_{i, \varepsilon, \zeta}^{n}\right|_{1}^{2}+\left|\sum_{i=1}^{2}\left(U_{i, \varepsilon, \zeta}^{n}-U_{i, \varepsilon}^{n-1}\right)\right|_{1}^{2}\right] \\
& +\left(\left[\sum_{i=1}^{2} \Phi_{i, \zeta}\left(U_{i, \varepsilon, \zeta}^{n}\right)\right]+\Phi_{3, \zeta}\left(\sum_{i=1}^{2} U_{i, \varepsilon, \zeta}^{n}\right), 1\right)^{h} \\
& \leq \frac{c_{1}}{2}\left|U_{1, \varepsilon}^{n-1}\right|_{1}^{2}+\frac{c_{2}}{2}\left|\sum_{i=1}^{2} U_{i, \varepsilon}^{n-1}\right|_{1}^{2}+\left(\left[\sum_{i=1}^{2} \Phi_{i, \zeta}\left(U_{i, \varepsilon}^{n-1}\right)\right]+\Phi_{3, \zeta}\left(\sum_{i=1}^{2} U_{i, \varepsilon}^{n-1}\right), 1\right)^{h}+\sum_{i=1}^{2}\left(W_{i, \varepsilon, \zeta}^{n}, U_{i, \varepsilon, \zeta}^{n}-U_{i, \varepsilon}^{n-1}\right)^{h} .
\end{aligned}
$$

Combining (2.37) and (2.35) yields for $\zeta \in\left(0, \zeta_{1}\right]$ that

$$
\begin{aligned}
& \frac{c_{1}}{2}\left[\left|U_{1, \varepsilon, \zeta}^{n}\right|_{1}^{2}+\left|U_{1, \varepsilon, \zeta}^{n}-U_{1, \varepsilon}^{n-1}\right|_{1}^{2}\right]+\frac{c_{2}}{2}\left[\left|\sum_{i=1}^{2} U_{i, \varepsilon, \zeta}^{n}\right|_{1}^{2}+\left|\sum_{i=1}^{2}\left(U_{i, \varepsilon, \zeta}^{n}-U_{i, \varepsilon}^{n-1}\right)\right|_{1}^{2}\right] \\
& +\left(\left[\sum_{i=1}^{2} \Phi_{i, \zeta}\left(U_{i, \varepsilon, \zeta}^{n}\right)\right]+\Phi_{3, \zeta}\left(\sum_{i=1}^{2} U_{i, \varepsilon, \zeta}^{n}\right), 1\right)^{h}+\frac{\tau}{3 \mu}\left(\left[\Xi_{M, \zeta}\left(U_{1, \varepsilon, \zeta}^{n}\right)\right]^{3} \nabla W_{1, \varepsilon, \zeta}^{n}, \nabla W_{1, \varepsilon, \zeta}^{n}\right) \\
& +\frac{\tau}{3}\left(\left[\Xi_{\zeta}\left(U_{2, \varepsilon, \zeta}^{n}\right)\right]^{3} \nabla W_{2, \varepsilon, \zeta}^{n}, \nabla W_{2, \varepsilon, \zeta}^{n}\right)+\frac{\tau}{\mu}\left(\Xi_{M, \zeta}\left(U_{1, \varepsilon, \zeta}^{n}\right)\left[\Xi_{\zeta}\left(U_{2, \varepsilon, \zeta}^{n}\right)\right]^{2} \nabla W_{2, \varepsilon, \zeta}^{n}, \nabla W_{2, \varepsilon, \zeta}^{n}\right) \\
& \leq \frac{c_{1}}{2}\left|U_{1, \varepsilon}^{n-1}\right|_{1}^{2}+\frac{c_{2}}{2}\left|\sum_{i=1}^{2} U_{i, \varepsilon}^{n-1}\right|_{1}^{2}+\left(\left[\sum_{i=1}^{2} \Phi_{i, \zeta}\left(U_{i, \varepsilon}^{n-1}\right)\right]+\Phi_{3, \zeta}\left(\sum_{i=1}^{2} U_{i, \varepsilon}^{n-1}\right), 1\right)^{h} \\
& \quad-\frac{\tau}{\mu}\left(\left[\Xi_{M, \zeta}\left(U_{1, \varepsilon, \zeta}^{n}\right)\right]^{2} \Xi_{\zeta}\left(U_{2, \varepsilon, \zeta}^{n}\right) \nabla W_{1, \varepsilon, \zeta}^{n}, \nabla W_{2, \varepsilon, \zeta}^{n}\right)-\frac{\tau}{2}\left(\left[\Xi_{\zeta}\left(U_{2, \varepsilon, \zeta}^{n}\right)\right]^{\frac{3}{2}}\left[\Xi\left(U_{2, \varepsilon}^{n-1}\right)\right]^{\frac{1}{2}} \nabla V_{2, \varepsilon}^{n-1}, \nabla W_{2, \varepsilon, \zeta}^{n}\right) \\
& \quad-\frac{\tau}{2 \mu}\left(\left[\Xi_{M, \zeta}\left(U_{1, \varepsilon, \zeta}^{n}\right)\right]^{\frac{3}{2}}\left[\Xi_{M}\left(U_{1, \varepsilon}^{n-1}\right)\right]^{\frac{1}{2}} \nabla\left[V_{1, \varepsilon}^{n-1}+V_{2, \varepsilon}^{n-1}\right], \nabla W_{1, \varepsilon, \zeta}^{n}\right) \\
& \quad-\frac{\tau}{\mu}\left(\left[\Xi_{M, \zeta}\left(U_{1, \varepsilon, \zeta}^{n}\right)\right]^{\frac{1}{2}}\left[\Xi_{M}\left(U_{1, \varepsilon}^{n-1}\right)\right]^{\frac{1}{2}} \Xi_{\zeta}\left(U_{2, \varepsilon, \zeta}^{n}\right) \nabla\left[V_{1, \varepsilon}^{n-1}+V_{2, \varepsilon}^{n-1}\right], \nabla W_{2, \varepsilon, \zeta}^{n}\right) .
\end{aligned}
$$

Applying Young's inequality, (1.16), to the right-hand side of (2.38), with $\gamma=\frac{7}{12}$ for the crucial fourth term, yields that it can be bounded by

$$
\begin{aligned}
\frac{15 \tau}{48 \mu}( & {\left.\left[\Xi_{M, \zeta}\left(U_{1, \varepsilon, \zeta}^{n}\right)\right]^{3} \nabla W_{1, \varepsilon, \zeta}^{n}, \nabla W_{1, \varepsilon, \zeta}^{n}\right)+\frac{\tau}{6}\left(\left[\Xi_{\zeta}\left(U_{2, \varepsilon, \zeta}^{n}\right)\right]^{3} \nabla W_{2, \varepsilon, \zeta}^{n}, \nabla W_{2, \varepsilon, \zeta}^{n}\right) } \\
& +\frac{13 \tau}{14 \mu}\left(\Xi_{M, \zeta}\left(U_{1, \varepsilon, \zeta}^{n}\right)\left[\Xi_{\zeta}\left(U_{2, \varepsilon, \zeta}^{n}\right)\right]^{2} \nabla W_{2, \varepsilon, \zeta}^{n}, \nabla W_{2, \varepsilon, \zeta}^{n}\right)+C\left(\tau, h, \mu,\left\{U_{i, \varepsilon}^{n-1}, V_{i, \varepsilon}^{n-1}\right\}_{i=1}^{2}\right) .
\end{aligned}
$$


Hence combining (2.38) and (2.39) yields the existence of constants $C_{k}$ independent of $\zeta \in\left(0, \zeta_{1}\right]$ such that for $i=1,2$

$$
\left(\Phi_{i, \zeta}\left(U_{i, \varepsilon, \zeta}^{n}\right), 1\right)^{h} \leq C_{1} \quad \Rightarrow \quad \Phi_{i, \zeta}\left(U_{i, \varepsilon, \zeta}^{n}\left(p_{j}\right)\right) \leq C_{2} h_{\min }^{-d}:=C_{3} \quad \forall j \in J
$$

where $h_{\min }:=\min _{\kappa \in \mathcal{T}^{h}} h_{\kappa}$. The bound (2.40), on noting (2.18) and (2.26), yields for all $\zeta \in\left(0, \zeta_{2}\right]$, where $\zeta_{2}=\min \left\{\Phi_{1}^{-1}\left(C_{3}\right), \Phi_{2}^{-1}\left(C_{3}\right), \zeta_{1}\right\}$, that for $i=1,2$

$$
\Phi_{i}\left(U_{i, \varepsilon, \zeta}^{n}\left(p_{j}\right)\right)=\Phi_{i, \zeta}\left(U_{i, \varepsilon, \zeta}^{n}\left(p_{j}\right)\right) \leq C_{3} \quad \Rightarrow \quad U_{i, \varepsilon, \zeta}^{n}\left(p_{j}\right) \geq \zeta_{2}>0 \quad \text { for all nodes } p_{j} \in \mathcal{T}^{h} .
$$

This yields that for all $\zeta \in\left(0, \zeta_{2}\right]$ a solution $\left\{U_{i, \varepsilon, \zeta}^{n}, W_{1, \varepsilon, \zeta}^{n}\right\}_{i=1}^{2}$ to $(2.28 \mathrm{a}-\mathrm{d})$ solves $(2.4 \mathrm{a}-\mathrm{d})$, since $\Xi_{M, \zeta}\left(U_{1, \varepsilon, \zeta}^{n}\right) \equiv$ $\Xi_{M}\left(U_{1, \varepsilon, \zeta}^{n}\right), \Xi_{\zeta}\left(U_{2, \varepsilon, \zeta}^{n}\right)=\Xi\left(U_{2, \varepsilon, \zeta}^{n}\right), \phi_{i, \zeta}^{ \pm}\left(U_{i, \zeta, \varepsilon}^{n}\right)=\phi_{i}^{ \pm}\left(U_{i, \zeta, \varepsilon}^{n}\right), i=1,2$, and $\phi_{3, \zeta}^{ \pm}\left(\sum_{i=1}^{2} U_{i, \zeta, \varepsilon}^{n}\right)=\phi_{3}^{ \pm}\left(\sum_{i=1}^{2} U_{i, \zeta, \varepsilon}^{n}\right)$.

We now address the simpler task of proving existence of a solution $\left\{V_{i, \varepsilon}^{n}\right\}_{i=1}^{2}$ to $(2.4 \mathrm{e}, \mathrm{f})$. Choosing $\chi=$ $\pi^{h}\left[F_{\varepsilon}^{\prime}\left(V_{i, \varepsilon}^{n}\right)\right], i=1,2$, in $(2.4 \mathrm{e}, \mathrm{f})$, respectively, and adding; yields, on noting (2.3b), that

$$
\begin{aligned}
& \mu \sum_{i=1}^{2}\left[\left(V_{i, \varepsilon}^{n}-V_{i, \varepsilon}^{n-1}, F_{\varepsilon}^{\prime}\left(V_{i, \varepsilon}^{n}\right)\right)^{h}+\tau \rho_{i}\left(\nabla V_{i, \varepsilon}^{n}, \nabla \pi^{h}\left[F_{\varepsilon}^{\prime}\left(V_{i, \varepsilon}^{n}\right)\right]\right)\right]+\tau \mu\left(\Xi\left(U_{2, \varepsilon}^{n}\right) \nabla V_{2, \varepsilon}^{n}, \nabla V_{2, \varepsilon}^{n}\right) \\
& +\tau\left(\Xi_{M}\left(U_{1, \varepsilon}^{n}\right) \nabla\left[\sum_{i=1}^{2} V_{i, \varepsilon}^{n}\right], \nabla\left[\sum_{i=1}^{2} V_{i, \varepsilon}^{n}\right]\right) \\
& \quad=-\tau\left(\frac{1}{2}\left[\Xi_{M}\left(U_{1, \varepsilon}^{n}\right)\right]^{2} \nabla W_{1, \varepsilon}^{n}+\Xi_{M}\left(U_{1, \varepsilon}^{n}\right) \Xi\left(U_{2, \varepsilon}^{n}\right) \nabla W_{2, \varepsilon}^{n}, \nabla\left[\sum_{i=1}^{2} V_{i, \varepsilon}^{n}\right]\right)-\frac{\tau \mu}{2}\left(\left[\Xi\left(U_{2, \varepsilon}^{n}\right)\right]^{2} \nabla W_{2, \varepsilon}^{n}, \nabla V_{2, \varepsilon}^{n}\right) .
\end{aligned}
$$

Now, $F_{\varepsilon}^{\prime \prime} \geq 1$ implies for $i=1,2$ that

$$
\left(V_{i, \varepsilon}^{n}-V_{i, \varepsilon}^{n-1}, F_{\varepsilon}^{\prime}\left(V_{i, \varepsilon}^{n}\right)\right)^{h} \geq\left(F_{\varepsilon}\left(V_{i, \varepsilon}^{n}\right)-F_{\varepsilon}\left(V_{i, \varepsilon}^{n-1}\right), 1\right)^{h}+\frac{1}{2}\left|V_{i, \varepsilon}^{n}-V_{i, \varepsilon}^{n-1}\right|_{h}^{2} .
$$

It follows from (2.42), (2.43), (1.12) and (2.12) that

$$
\sum_{i=1}^{2}\left|V_{i, \varepsilon}^{n}\right|_{h}^{2} \leq C\left(\tau, \mu,\left\{\rho_{i}, V_{i, \varepsilon}^{n-1}, U_{i, \varepsilon}^{n}, W_{i, \varepsilon}^{n}\right\}_{i=1}^{2}\right) .
$$

On noting the above, and recalling Lemma 2.3 and that $\left[F_{\varepsilon}^{\prime}(\cdot)\right]^{-1}$ is uniquely defined on $\mathbb{R}$, it follows that $(2.4 \mathrm{e}, \mathrm{f})$ is a continuous and coercive discrete nonlinear system. The Brouwer fixed point theorem then yields the existence of a solution $\left\{V_{i, \varepsilon}^{n}\right\}_{i=1}^{2}$ to $(2.4 \mathrm{e}, \mathrm{f})$. 
Lemma 2.5. Let the assumptions of Theorem 2.4 hold. Then for all $\varepsilon \in(0,1)$ and for all $h, \tau>0$ a solution $\left\{U_{i, \varepsilon}^{n}, W_{i, \varepsilon}^{n}, V_{i, \varepsilon}^{n}\right\}_{i=1}^{2}$ to the nth step of $\left(\mathrm{P}_{\varepsilon}^{h, \tau}\right)$ is such that

$$
\begin{gathered}
\mathcal{E}\left(\left\{U_{i, \varepsilon}^{n}, V_{i, \varepsilon}^{n}\right\}_{i=1}^{2}\right)+\frac{1}{2}\left[c_{1}\left|U_{1, \varepsilon}^{n}-U_{1, \varepsilon}^{n-1}\right|_{1}^{2}+c_{2}\left|\sum_{i}^{2}\left[U_{i, \varepsilon}^{n}-U_{i, \varepsilon}^{n-1}\right]\right|_{1}^{2}+\sum_{i=2}^{2}\left|V_{i, \varepsilon}^{n}-V_{i, \varepsilon}^{n-1}\right|_{h}^{2}\right] \\
+\tau\left[\sum_{i=1}^{2} \rho_{i}\left(\nabla V_{i, \varepsilon}^{n}, \nabla \pi^{h}\left[F_{\varepsilon}^{\prime}\left(V_{i, \varepsilon}^{n}\right)\right]\right)\right]+\frac{\tau}{24}\left[\frac{1}{\mu}\left|\left[\Xi_{M}\left(U_{1, \varepsilon}^{n}\right)\right]^{\frac{3}{2}} \nabla W_{1, \varepsilon}^{n}\right|_{0}^{2}+\left|\left[\Xi\left(U_{2, \varepsilon}^{n}\right)\right]^{\frac{3}{2}} \nabla W_{2, \varepsilon}^{n}\right|_{0}^{2}\right] \\
+\frac{4 \tau}{7}\left|\left[\Xi\left(U_{2, \varepsilon}^{n}\right)\right]^{\frac{1}{2}} \nabla V_{2, \varepsilon}^{n}\right|_{0}^{2}+\frac{\tau}{14 \mu}\left|\left[\Xi_{M}\left(U_{1, \varepsilon}^{n}\right)\right]^{\frac{1}{2}} \Xi\left(U_{2, \varepsilon}^{n}\right) \nabla W_{2, \varepsilon}^{n}+\left[\Xi_{M}\left(U_{1, \varepsilon}^{n}\right)\right]^{\frac{1}{2}} \nabla\left[\sum_{i=1}^{2} V_{i, \varepsilon}^{n}\right]\right|_{0}^{2} \\
\left.+\frac{\tau}{2 \mu}\left|\left[\Xi_{M}\left(U_{1, \varepsilon}^{n}\right)\right]^{\frac{1}{2}} \nabla\left[\sum_{i=1}^{2} V_{i, \varepsilon}^{n}\right]\right|_{0}^{2}+\frac{\tau}{14 \mu} \mid\left[\Xi_{M}\left(U_{1, \varepsilon}^{n}\right)\right]^{\frac{1}{2}} \Xi\left(U_{2, \varepsilon}^{n}\right) \nabla W_{2, \varepsilon}^{n}+\left[\Xi_{M}\left(U_{1, \varepsilon}^{n-1}\right)\right]^{\frac{1}{2}} \nabla\left[\sum_{i=1}^{2} V_{i, \varepsilon}^{n-1}\right]\right]\left.\right|_{0} ^{2} \\
\leq \mathcal{E}\left(\left\{U_{i, \varepsilon}^{n-1}, V_{i, \varepsilon}^{n-1}\right\}_{i=1}^{2}\right)+\frac{3 \tau}{7}\left|\left[\Xi\left(U_{2, \varepsilon}^{n-1}\right)\right]^{\frac{1}{2}} \nabla V_{2, \varepsilon}^{n-1}\right|_{0}^{2}+\frac{\tau}{2 \mu}\left|\left[\Xi_{M}\left(U_{1, \varepsilon}^{n-1}\right)\right]^{\frac{1}{2}} \nabla\left[\sum_{i=1}^{2} V_{i, \varepsilon}^{n-1}\right]\right|_{0}^{2}, \quad(2.44)
\end{gathered}
$$

where

$$
\mathcal{E}\left(\left\{U_{i, \varepsilon}^{n}, V_{i, \varepsilon}^{n}\right\}_{i=1}^{2}\right):=\frac{1}{2}\left[c_{1}\left|U_{1, \varepsilon}^{n}\right|_{1}^{2}+c_{2}\left|\sum_{i=1}^{2} U_{i, \varepsilon}^{n}\right|_{1}^{2}\right]+\left(\sum_{i=1}^{2}\left[\Phi_{i}\left(U_{i, \varepsilon}^{n}\right)+F_{\varepsilon}\left(V_{i, \varepsilon}^{n}\right)\right]+\Phi_{3}\left(\sum_{i=1}^{2} U_{i, \varepsilon}^{n}\right), 1\right)^{h}
$$

Furthermore, if $\phi_{j}(\cdot)$ satisfies (1.3) with $\nu_{j} \geq 7, j=1,2$, then

$$
\begin{aligned}
\left(\mu G_{M}\left(U_{1, \varepsilon}^{n}\right)+G\left(U_{2, \varepsilon}^{n}\right), 1\right)^{h}+\frac{\tau}{3}\left[c_{1}\left|\Delta^{h} U_{1, \varepsilon}^{n}\right|_{h}^{2}+c_{2}\left|\Delta^{h}\left(\sum_{i=1}^{2} U_{i, \varepsilon}\right)\right|_{h}^{2}\right] \\
+\frac{\tau}{4}\left[\sum_{i=1}^{2}\left(\nabla \pi^{h}\left[\phi_{i}^{+}\left(U_{i, \varepsilon}^{n}\right)\right], \nabla U_{i, \varepsilon}^{n}\right)\right]+\frac{\tau}{4}\left(\nabla \pi^{h}\left[\phi_{3}^{+}\left(\sum_{i=1}^{2} U_{i, \varepsilon}^{n}\right)\right], \nabla\left[\sum_{i=1}^{2} U_{i, \varepsilon}^{n}\right]\right) \\
\leq\left(\mu G_{M}\left(U_{1, \varepsilon}^{n-1}\right)+G\left(U_{2, \varepsilon}^{n-1}\right), 1\right)^{h}+\frac{\tau}{8}\left[\sum_{i=1}^{2}\left(\nabla \pi^{h}\left[\phi_{i}^{+}\left(U_{i, \varepsilon}^{n-1}\right)\right], \nabla U_{i, \varepsilon}^{n-1}\right)\right] \\
+\frac{1}{4}\left|\left[\Xi\left(U_{2, \varepsilon}^{n-1}\right)\right]^{\frac{1}{2}} \nabla V_{2, \varepsilon}^{n-1}\right|_{0}^{2}+C(M)\left|\left[\Xi_{M}\left(U_{1, \varepsilon}^{n}\right)\right]^{\frac{3}{2}} \nabla W_{1, \varepsilon}^{n}\right|_{0}^{2}+C \sum_{i=1}^{2}\left[\left|U_{i, \varepsilon}^{n}\right|_{1}^{2}+\left|U_{i, \varepsilon}^{n-1}\right|_{1}^{2}\right] \\
+C(M)\left|\left[\Xi_{M}\left(U_{1, \varepsilon}^{n}\right)\right]^{\frac{1}{2}} \Xi\left(U_{2, \varepsilon}^{n}\right) \nabla W_{2, \varepsilon}^{n}+\left[\Xi_{M}\left(U_{1, \varepsilon}^{n-1}\right)\right]^{\frac{1}{2}} \nabla\left[\sum_{i=1}^{2} V_{i, \varepsilon}^{n-1}\right]\right|_{0}^{2}
\end{aligned}
$$

Proof. Choosing $\chi=W_{i, \varepsilon}^{n}, i=1,2$, in (2.4a), respectively, $\chi=U_{1, \varepsilon}^{n}-U_{1, \varepsilon}^{n-1}$ in $(2.4 \mathrm{c}), \chi=\sum_{i=1}^{2}\left[U_{i, \varepsilon}^{n}-U_{i, \varepsilon}^{n-1}\right]$ in (2.4d), and combining; yields the $\zeta$ unregularized version of (2.38). Combining this with (2.42) yields, 
on noting (2.43) and (2.45), and applying Young's inequality (1.16), that

$$
\begin{aligned}
& \mathcal{E}\left(\left\{U_{i, \varepsilon}^{n}, V_{i, \varepsilon}^{n}\right\}_{i=1}^{2}\right)+\frac{1}{2}\left[c_{1}\left|U_{1, \varepsilon}^{n}-U_{1, \varepsilon}^{n-1}\right|_{1}^{2}+c_{2}\left|\sum_{i}^{2}\left[U_{i, \varepsilon}^{n}-U_{i, \varepsilon}^{n-1}\right]\right|_{1}^{2}+\sum_{i=2}^{2}\left|V_{i, \varepsilon}^{n}-V_{i, \varepsilon}^{n-1}\right|_{h}^{2}\right] \\
&\left.+\tau \mid \sum_{i=1}^{2} \rho_{i}\left(\nabla V_{i, \varepsilon}^{n}, \nabla \pi^{h}\left[F_{\varepsilon}^{\prime}\left(V_{i, \varepsilon}^{n}\right)\right]\right)\right]+\frac{\tau}{3}\left[\frac{1}{\mu}\left|\left[\Xi_{M}\left(U_{1, \varepsilon}^{n}\right)\right]^{\frac{3}{2}} \nabla W_{1, \varepsilon}^{n}\right|_{0}^{2}+\left|\left[\Xi\left(U_{2, \varepsilon}^{n}\right)\right]^{\frac{3}{2}} \nabla W_{2, \varepsilon}^{n}\right|_{0}^{2}\right] \\
&+\tau\left|\left[\Xi\left(U_{2, \varepsilon}^{n}\right)\right]^{\frac{1}{2}} \nabla V_{2, \varepsilon}^{n}\right|_{0}^{2}+\frac{\tau}{2 \mu}\left|\left[\Xi_{M}\left(U_{1, \varepsilon}^{n}\right)\right]^{\frac{1}{2}} \Xi\left(U_{2, \varepsilon}^{n}\right) \nabla W_{2, \varepsilon}^{n}+\left[\Xi_{M}\left(U_{1, \varepsilon}^{n}\right)\right]^{\frac{1}{2}} \nabla\left[\sum_{i=1}^{2} V_{i, \varepsilon}^{n}\right]\right|_{0}^{2} \\
&\left.+\frac{\tau}{2 \mu}\left|\left[\Xi_{M}\left(U_{1, \varepsilon}^{n}\right)\right]^{\frac{1}{2}} \nabla\left[\sum_{i=1}^{2} V_{i, \varepsilon}^{n}\right]\right|_{0}^{2}+\frac{\tau}{2 \mu} \mid\left[\Xi_{M}\left(U_{1, \varepsilon}^{n}\right)\right]^{\frac{1}{2}} \Xi\left(U_{2, \varepsilon}^{n}\right) \nabla W_{2, \varepsilon}^{n}+\left[\Xi_{M}\left(U_{1, \varepsilon}^{n-1}\right)\right]\right]\left.^{\frac{1}{2}} \nabla\left[\sum_{i=1}^{2} V_{i, \varepsilon}^{n-1}\right]\right|_{0} ^{2} \\
& \leq \mathcal{E}\left(\left\{U_{i, \varepsilon}^{n-1}, V_{i, \varepsilon}^{n-1}\right\}_{i=1}^{2}\right)+\frac{\tau}{2 \mu}\left|\left[\Xi_{M}\left(U_{1, \varepsilon}^{n-1}\right)\right]^{\frac{1}{2}} \nabla\left[\sum_{i=1}^{2} V_{i, \varepsilon}^{n-1}\right]\right|_{0}^{2}-\frac{\tau}{2}\left(\left[\Xi\left(U_{2, \varepsilon}^{n}\right)\right]^{2} \nabla W_{2, \varepsilon}^{n}, \nabla V_{2, \varepsilon}^{n}\right) \\
&-\frac{\tau}{2 \mu}\left(\left[\Xi_{M}\left(U_{1, \varepsilon}^{n}\right)\right]^{\frac{3}{2}} \nabla W_{1, \varepsilon}^{n},\left[\Xi_{M}\left(U_{1, \varepsilon}^{n}\right)\right]^{\frac{1}{2}} \Xi\left(U_{2, \varepsilon}^{n}\right) \nabla W_{2, \varepsilon}^{n}+\left[\Xi_{M}\left(U_{1, \varepsilon}^{n-1}\right)\right]^{\frac{1}{2}} \nabla\left[\sum_{i=1}^{2} V_{i, \varepsilon}^{n-1}\right]\right) \\
&\left.\left.-\frac{\tau}{2}\left(\left[\Xi\left(U_{2, \varepsilon}^{n}\right)\right]^{\frac{3}{2}}\left[\Xi\left(U_{2, \varepsilon}^{n-1}\right)\right]^{\frac{1}{2}} \nabla W_{2, \varepsilon}^{n}, \nabla V_{2, \varepsilon}^{n-1}\right)-\frac{\tau}{2 \mu}\left(\left[\Xi_{M}\left(U_{1, \varepsilon}^{n}\right)\right]^{2} \nabla W_{1, \varepsilon}^{n}, \Xi\left(U_{2, \varepsilon}^{n}\right) \nabla W_{2, \varepsilon}^{n}+\nabla\right] \sum_{i=1}^{2} V_{i, \varepsilon}^{n}\right]\right) \\
& \leq \mathcal{E}\left(\left\{U_{i, \varepsilon}^{n-1}, V_{i, \varepsilon}^{n-1}\right\}_{i=1}^{2}\right)+\frac{\tau}{2 \mu}\left|\left[\Xi_{M}\left(U_{1, \varepsilon}^{n-1}\right)\right]^{\frac{1}{2}} \nabla\left[\sum_{i=1}^{2} V_{i, \varepsilon}^{n-1}\right]\right|_{0}^{2}+\frac{\tau}{4 \gamma}\left|\left[\Xi\left(U_{2, \varepsilon}^{n-1}\right)\right]^{\frac{1}{2}} \nabla V_{2, \varepsilon}^{n-1}\right|_{0}^{2} \\
&+\frac{\tau \gamma}{2}\left[\frac{1}{\mu}\left|\left[\Xi_{M}\left(U_{1, \varepsilon}^{n}\right)\right]^{\frac{3}{2}} \nabla W_{1, \varepsilon}^{n}\right|_{0}^{2}+\left|\left[\Xi\left(U_{2, \varepsilon}^{n}\right)\right]^{\frac{3}{2}} \nabla W_{2, \varepsilon}^{n}\right|_{0}^{2}\right]+\frac{\tau}{4 \gamma}\left|\left[\Xi\left(U_{2, \varepsilon}^{n}\right)\right]^{\frac{1}{2}} \nabla V_{2, \varepsilon}^{n}\right|_{0}^{2} \\
&+\frac{\tau}{4 \gamma \mu}\left|\left[\Xi_{M}\left(U_{1, \varepsilon}^{n}\right)\right]^{\frac{1}{2}} \Xi\left(U_{2, \varepsilon}^{n}\right) \nabla W_{2, \varepsilon}^{n}+\left[\Xi_{M}\left(U_{1, \varepsilon}^{n}\right)\right]^{\frac{1}{2}} \nabla\left[\sum_{i=1}^{2} V_{i, \varepsilon}^{n}\right]\right|_{0}^{2} \\
&+\left.\frac{\tau}{4 \gamma \mu}\left|\left[\Xi_{M}\left(U_{1, \varepsilon}^{n}\right)\right]^{\frac{1}{2}} \Xi\left(U_{2, \varepsilon}^{n}\right) \nabla W_{2, \varepsilon}^{n}+\left[\Xi_{M}\left(U_{1, \varepsilon}^{n-1}\right)\right]^{\frac{1}{2}} \nabla\left[\sum_{i=1}^{2} V_{i, \varepsilon}^{n-1}\right]\right|\right|_{0} ^{2} .
\end{aligned}
$$

Hence, on choosing $\gamma=\frac{7}{12}$ in (2.47) yields the desired result (2.44).

Choosing $\chi=\pi^{h}\left[G_{M}^{\prime}\left(U_{1, \varepsilon}^{n}\right)\right]$ in (2.4a), and noting (2.3b), yields that

$$
\begin{aligned}
\mu & \left(\frac{U_{1, \varepsilon}^{n}-U_{1, \varepsilon}^{n-1}}{\tau}, G_{M}^{\prime}\left(U_{1, \varepsilon}^{n}\right)\right)^{h}+\frac{1}{3}\left(\nabla W_{1, \varepsilon}^{n}, \nabla U_{1, \varepsilon}^{n}\right) \\
& =-\frac{1}{2}\left(\left[\Xi_{M}\left(U_{1, \varepsilon}^{n}\right)\right]^{2} \Xi\left(U_{2, \varepsilon}^{n}\right) \nabla W_{2, \varepsilon}^{n}+\left[\Xi_{M}\left(U_{1, \varepsilon}^{n}\right)\right]^{\frac{3}{2}}\left[\Xi_{M}\left(U_{1, \varepsilon}^{n-1}\right)\right]^{\frac{1}{2}} \nabla\left[\sum_{i=1}^{2} V_{i, \varepsilon}^{n-1}\right], \nabla \pi^{h}\left[G_{M}^{\prime}\left(U_{1, \varepsilon}^{n}\right)\right]\right) \\
& \leq \frac{1}{4}\left|\left[\Xi_{M}\left(U_{1, \varepsilon}^{n}\right)\right]^{\frac{1}{2}} \Xi\left(U_{2, \varepsilon}^{n}\right) \nabla W_{2, \varepsilon}^{n}+\left[\Xi_{M}\left(U_{1, \varepsilon}^{n-1}\right)\right]^{\frac{1}{2}} \nabla\left[\sum_{i=1}^{2} V_{i, \varepsilon}^{n-1}\right]\right|_{0}^{2}+\frac{1}{4}\left(\nabla \pi^{h}\left[G_{M}^{\prime}\left(U_{1, \varepsilon}^{n}\right)\right], \nabla U_{1, \varepsilon}^{n}\right) .
\end{aligned}
$$


Similarly, on choosing $\chi=\pi^{h}\left[G^{\prime}\left(U_{2, \varepsilon}^{n}\right)\right]$ in (2.4b), yields that for all $\gamma>0$, there exists $C(\gamma, M, \mu)>0$ such that

$$
\begin{aligned}
& \left(\frac{U_{2, \varepsilon}^{n}-U_{2, \varepsilon}^{n-1}}{\tau}, G^{\prime}\left(U_{2, \varepsilon}^{n}\right)\right)^{h}+\frac{1}{3}\left(\nabla W_{2, \varepsilon}^{n}, \nabla U_{2, \varepsilon}^{n}\right) \\
& \leq \frac{1}{4}\left[\left|\left[\Xi\left(U_{2, \varepsilon}^{n-1}\right)\right]^{\frac{1}{2}} \nabla V_{2, \varepsilon}^{n-1}\right|_{0}^{2}+\left(\nabla \pi^{h}\left[G^{\prime}\left(U_{2, \varepsilon}^{n}\right)\right], \nabla U_{2, \varepsilon}^{n}\right)\right]+C(\gamma, M, \mu)\left|\left[\Xi_{M}\left(U_{1, \varepsilon}^{n}\right)\right]^{\frac{3}{2}} \nabla W_{1, \varepsilon}^{n}\right|_{0}^{2} \\
& \quad+C(\gamma, M, \mu)\left|\left[\Xi_{M}\left(U_{1, \varepsilon}^{n}\right)\right]^{\frac{1}{2}} \Xi\left(U_{2, \varepsilon}^{n}\right) \nabla W_{2, \varepsilon}^{n}+\left[\Xi_{M}\left(U_{1, \varepsilon}^{n-1}\right)\right]^{\frac{1}{2}} \nabla\left[\sum_{i=1}^{2} V_{i, \varepsilon}^{n-1}\right]\right|_{0}^{2}+\gamma\left|\Xi\left(U_{2, \varepsilon}^{n}\right) \nabla \pi^{h}\left[G^{\prime}\left(U_{2, \varepsilon}^{n}\right)\right]\right|_{0}^{2} .
\end{aligned}
$$

Choosing $\chi=-\Delta^{h} U_{1, \varepsilon}^{n}$ in (2.4c) and $\chi=-\Delta^{h}\left[\sum_{i=1}^{2} U_{i, \varepsilon}^{n}\right]$ in (2.4d), then adding yields, on noting (2.14) and (2.1), that

$$
\begin{aligned}
\sum_{i=1}^{2}\left(\nabla W_{i, \varepsilon}^{n}, \nabla U_{i, \varepsilon}^{n}\right)=c_{1}\left|\Delta^{h} U_{1, \varepsilon}^{n}\right|_{h}^{2}+c_{2} \mid \Delta^{h} & {\left.\left[\sum_{i=1}^{2} U_{i, \varepsilon}^{n}\right]\right|_{h} ^{2}+\sum_{i=1}^{2}\left(\nabla \pi^{h}\left[\phi_{i}^{+}\left(U_{i, \varepsilon}^{n}\right)+\phi_{i}^{-}\left(U_{i, \varepsilon}^{n-1}\right)\right], \nabla U_{i, \varepsilon}^{n}\right) } \\
& +\left(\nabla \pi^{h}\left[\phi_{3}^{+}\left(\sum_{i=1}^{2} U_{i, \varepsilon}^{n}\right)+\phi_{3}^{-}\left(\sum_{i=1}^{2} U_{i, \varepsilon}^{n-1}\right)\right], \nabla\left[\sum_{i=1}^{2} U_{i, \varepsilon}^{n}\right]\right) .
\end{aligned}
$$

Combining (2.48)-(2.50), and noting the convexity of $G_{(M)},(2.20),(2.22),(2.24)$ and (2.25), it follows for all $\gamma>0$ that

$$
\begin{aligned}
& \mu\left(G_{M}\left(U_{1, \varepsilon}^{n}\right)-G_{M}\left(U_{1, \varepsilon}^{n-1}\right), 1\right)^{h}+\left(G\left(U_{2, \varepsilon}^{n}\right)-G\left(U_{2, \varepsilon}^{n-1}\right), 1\right)^{h}+\frac{\tau}{3}\left[c_{1}\left|\Delta^{h} U_{1, \varepsilon}^{n}\right|_{h}^{2}+c_{2}\left|\Delta^{h}\left[\sum_{i=1}^{2} U_{i, \varepsilon}^{n}\right]\right|_{h}^{2}\right] \\
& +\tau\left(\frac{1}{3}-\gamma\right)\left[\sum_{i=1}^{2}\left(\nabla \pi^{h}\left[\phi_{i}^{+}\left(U_{i, \varepsilon}^{n}\right)\right], \nabla U_{i, \varepsilon}^{n}\right)\right]+\frac{\tau}{3}\left(\nabla \pi^{h}\left[\phi_{3}^{+}\left(\sum_{i=1}^{2} U_{i, \varepsilon}^{n}\right)\right], \nabla\left[\sum_{i=1}^{2} U_{i, \varepsilon}^{n}\right]\right) \\
& \leq \frac{1}{4}\left|\left[\Xi\left(U_{2, \varepsilon}^{n-1}\right)\right]^{\frac{1}{2}} \nabla V_{2, \varepsilon}^{n-1}\right|_{0}^{2}+C(\gamma, M, \mu)\left|\left[\Xi_{M}\left(U_{1, \varepsilon}^{n}\right)\right]^{\frac{1}{2}} \Xi\left(U_{2, \varepsilon}^{n}\right) \nabla W_{2, \varepsilon}^{n}+\left[\Xi_{M}\left(U_{1, \varepsilon}^{n-1}\right)\right]^{\frac{1}{2}} \nabla\left[\sum_{i=1}^{2} V_{i, \varepsilon}^{n-1}\right]\right|_{0}^{2} \\
& +C(\gamma, M, \mu)\left|\left[\Xi_{M}\left(U_{1, \varepsilon}^{n}\right)\right]^{\frac{3}{2}} \nabla W_{1, \varepsilon}^{n}\right|_{0}^{2}+C\left(\gamma,\left\{a_{j}, \delta_{j}, \nu_{j}\right\}_{j=1}^{3}\right) \sum_{i=1}^{2}\left[\left|U_{i, \varepsilon}^{n}\right|_{1}^{2}+\left|U_{i, \varepsilon}^{n-1}\right|_{1}^{2}\right] \\
& \quad+\frac{\tau}{8}\left[\sum_{i=1}^{2}\left(\nabla \pi^{h}\left[\phi_{i}^{+}\left(U_{i, \varepsilon}^{n-1}\right)\right], \nabla U_{i, \varepsilon}^{n-1}\right)\right]
\end{aligned}
$$

Choosing $\gamma$ sufficiently small yields the desired result (2.46).

We now prove discrete analogues of the formal energy bounds (1.15), with (1.17a,b), and (1.22).

Theorem 2.6. Let $\phi_{j}(\cdot)$ satisfy (1.3) with $\delta_{j}>0, j=1,2$. Let the assumptions (A) hold and $\left\{U_{i, \varepsilon}^{0}, V_{i, \varepsilon}^{0}\right\} \in$ $S_{>0}^{h} \times S^{h}$ for $i=1,2$. Then for all $\varepsilon \in(0,1), h>0$ and $\tau>0$ a solution $\left\{\left\{U_{i, \varepsilon}^{n}, W_{i, \varepsilon}^{n}, V_{i, \varepsilon}^{n}\right\}_{i=1}^{2}\right\}_{n=1}^{N}$ to $\left(\mathrm{P}_{\varepsilon}^{h, \tau}\right)$ 
is such that, for $i=1,2, U_{i, \varepsilon}^{n} \in S_{>0}^{h}, f U_{i, \varepsilon}^{n}=f U_{i, \varepsilon}^{0}$ and $f V_{i, \varepsilon}^{n}=f V_{i, \varepsilon}^{0}, n=1 \rightarrow N$, and

$$
\begin{aligned}
& c_{1} \max _{n=1 \rightarrow N}\left\|U_{1, \varepsilon}^{n}\right\|_{1}^{2}+c_{2} \max _{n=1 \rightarrow N}\left\|\sum_{i=1}^{2} U_{i, \varepsilon}^{n}\right\|_{1}^{2}+\sum_{i=1}^{2}\left[\max _{n=1 \rightarrow N}\left(\Phi_{i}\left(U_{i, \varepsilon}^{n}\right), 1\right)^{h}+\max _{n=1 \rightarrow N}\left(F_{\varepsilon}\left(V_{i, \varepsilon}^{n}\right), 1\right)^{h}\right] \\
& \quad+\max _{n=1 \rightarrow N}\left(\Phi_{3}\left(\sum_{i=1}^{2} U_{i, \varepsilon}^{n}\right), 1\right)^{h}+\sum_{i=1}^{2}\left[\max _{n=1 \rightarrow N}\left|V_{i, \varepsilon}^{n}\right|_{0}^{2}+\varepsilon^{-1} \max _{n=1 \rightarrow N}\left|\pi^{h}\left[V_{i, \varepsilon}^{n}\right]-\right|_{0}^{2}\right]+c_{1} \sum_{n=1}^{N}\left\|U_{1, \varepsilon}^{n}-U_{1, \varepsilon}^{n-1}\right\|_{1}^{2} \\
& \quad+c_{2} \sum_{n=1}^{N}\left\|\sum_{i=1}^{2}\left[U_{i, \varepsilon}^{n}-U_{i, \varepsilon}^{n-1}\right]\right\|_{1}^{2}+\sum_{i=1}^{2}\left[\sum_{n=1}^{N}\left|V_{i, \varepsilon}^{n}-V_{i, \varepsilon}^{n-1}\right|_{0}^{2}+\rho_{i} \sum_{n=1}^{N} \tau\left(\nabla V_{i, \varepsilon}^{n}, \nabla \pi^{h}\left[F_{\varepsilon}^{\prime}\left(V_{i, \varepsilon}^{n}\right)\right]\right)+\rho_{i} \sum_{n=1}^{N} \tau\left\|V_{i, \varepsilon}^{n}\right\|_{1}^{2}\right] \\
& \quad+\sum_{n=1}^{N} \tau\left[\mu^{-1}\left|\left[\Xi_{M}\left(U_{1, \varepsilon}^{n}\right)\right]^{\frac{3}{2}} \nabla W_{1, \varepsilon}^{n}\right|_{0}^{2}+\left|\left[\Xi\left(U_{2, \varepsilon}^{n}\right)\right]^{\frac{3}{2}} \nabla W_{2, \varepsilon}^{n}\right|_{0}^{2}+\mu^{-1}\left|\left[\Xi_{M}\left(U_{1, \varepsilon}^{n}\right)\right]^{\frac{1}{2}} \Xi\left(U_{2, \varepsilon}^{n}\right) \nabla W_{2, \varepsilon}^{n}\right|_{0}^{2}\right] \leq C \mathcal{C}^{0},
\end{aligned}
$$

where

$$
\begin{aligned}
\mathcal{C}^{0}:=1+\sum_{i=1}^{2}\left[\left[\left\|U_{i, \varepsilon}^{0}\right\|_{1}^{2}+\left(\Phi_{i}\left(U_{i, \varepsilon}^{0}\right)\right.\right.\right. & \left.\left.+F_{\varepsilon}\left(V_{i, \varepsilon}^{0}\right), 1\right)^{h}\right]+\left(\Phi_{3}\left(\sum_{i=1}^{2} U_{i, \varepsilon}^{0}\right), 1\right)^{h} \\
& \left.+\tau\left|\left[\Xi\left(U_{2, \varepsilon}^{0}\right)\right]^{\frac{1}{2}} \nabla V_{2, \varepsilon}^{0}\right|_{0}^{2}+\mu^{-1} \tau\left|\left[\Xi_{M}\left(U_{1, \varepsilon}^{0}\right)\right]^{\frac{1}{2}} \nabla\left[\sum_{i=1}^{2} V_{i, \varepsilon}^{0}\right]\right|_{0}^{2}\right] .
\end{aligned}
$$

In addition, we have that

$$
\sum_{n=1}^{N} \tau \sum_{i=1}^{2}\left[\left\|\mathcal{G}\left[\frac{U_{i, \varepsilon}^{n}-U_{i, \varepsilon}^{n-1}}{\tau}\right]\right\|_{1, q}^{2}+\left\|\mathcal{G}\left[\frac{V_{i, \varepsilon}^{n}-V_{i, \varepsilon}^{n-1}}{\tau}\right]\right\|_{1, q}^{2}\right] \leq C\left(M, \max _{n=0 \rightarrow N}\left\{\mid\left[\Xi\left(U_{2, \varepsilon}^{n}\right)\right]^{3}{ }_{0, r}\right\}\right) \mathcal{C}^{0},
$$

where $q=2$ and $r=\infty$ if $d=1, q \in(1,2)$ and $r=\frac{2 q}{2-q}$ if $d=2$; and

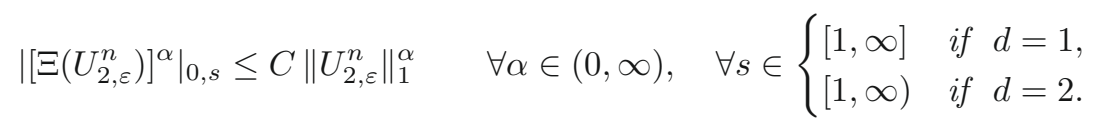

Furthermore if $\phi_{j}(\cdot)$ satisfies (1.3) with $\nu_{j} \geq 7, j=1,2$, then

$$
\begin{aligned}
& \mu \max _{n=1 \rightarrow N}\left(G_{M}\left(U_{1, \varepsilon}^{n}\right), 1\right)^{h}+\max _{n=1 \rightarrow N}\left(G\left(U_{2, \varepsilon}^{n}\right), 1\right)^{h}+\sum_{n=1}^{N} \tau\left[c_{1}\left|\Delta^{h} U_{1, \varepsilon}^{n}\right|_{h}^{2}+c_{2}\left|\Delta^{h}\left(\sum_{i=1}^{2} U_{i, \varepsilon}\right)\right|_{h}^{2}\right] \\
& +\sum_{n=1}^{N} \tau\left[\sum_{i=1}^{2}\left(\nabla \pi^{h}\left[\phi_{i}^{+}\left(U_{i, \varepsilon}^{n}\right)\right], \nabla U_{i, \varepsilon}^{n}\right)\right]+\sum_{n=1}^{N} \tau\left(\nabla \pi^{h}\left[\phi_{3}^{+}\left(\sum_{i=1}^{2} U_{i, \varepsilon}^{n}\right)\right], \nabla\left[\sum_{i=1}^{2} U_{i, \varepsilon}^{n}\right]\right) \\
& \quad \leq C\left[\mathcal{C}^{0}+\left(\mu G_{M}\left(U_{1, \varepsilon}^{0}\right)+G\left(U_{2, \varepsilon}^{0}\right), 1\right)^{h}+\tau \sum_{i=1}^{2}\left(\nabla \pi^{h}\left[\phi_{i}^{+}\left(U_{i, \varepsilon}^{0}\right)\right], \nabla U_{i, \varepsilon}^{0}\right)\right] .
\end{aligned}
$$


Proof. Summing (2.44) from $n=1 \rightarrow k$ yields for any $k \leq N$ that

$$
\begin{aligned}
& \mathcal{E}\left(\left\{U_{i, \varepsilon}^{k}, V_{i, \varepsilon}^{k}\right\}_{i=1}^{2}\right)+\frac{1}{2} \sum_{n=1}^{k}\left[c_{1}\left|U_{1, \varepsilon}^{n}-U_{1, \varepsilon}^{n-1}\right|_{1}^{2}+c_{2}\left|\sum_{i}^{2}\left[U_{i, \varepsilon}^{n}-U_{i, \varepsilon}^{n-1}\right]\right|_{1}^{2}+\sum_{i=2}^{2}\left|V_{i, \varepsilon}^{n}-V_{i, \varepsilon}^{n-1}\right|_{h}^{2}\right] \\
& +\sum_{n=1}^{k} \tau\left[\sum_{i=1}^{2} \rho_{i}\left(\nabla V_{i, \varepsilon}^{n}, \nabla \pi^{h}\left[F_{\varepsilon}^{\prime}\left(V_{i, \varepsilon}^{n}\right)\right]\right)\right]+\frac{1}{24} \sum_{n=1}^{k} \tau\left[\frac{1}{\mu}\left|\left[\Xi_{M}\left(U_{1, \varepsilon}^{n}\right)\right]^{\frac{3}{2}} \nabla W_{1, \varepsilon}^{n}\right|_{0}^{2}+\left|\left[\Xi\left(U_{2, \varepsilon}^{n}\right)\right]^{\frac{3}{2}} \nabla W_{2, \varepsilon}^{n}\right|_{0}^{2}\right] \\
& +\frac{1}{7} \sum_{n=1}^{k} \tau\left|\left[\Xi\left(U_{2, \varepsilon}^{n}\right)\right]^{\frac{1}{2}} \nabla V_{2, \varepsilon}^{n}\right|_{0}^{2}+\frac{1}{14 \mu} \sum_{n=1}^{k} \tau\left|\left[\Xi_{M}\left(U_{1, \varepsilon}^{n}\right)\right]^{\frac{1}{2}} \Xi\left(U_{2, \varepsilon}^{n}\right) \nabla W_{2, \varepsilon}^{n}+\left[\Xi_{M}\left(U_{1, \varepsilon}^{n}\right)\right]^{\frac{1}{2}} \nabla\left[\sum_{i=1}^{2} V_{i, \varepsilon}^{n}\right]\right|_{0}^{2} \\
& +\frac{1}{14 \mu} \sum_{n=1}^{k} \tau\left|\left[\Xi_{M}\left(U_{1, \varepsilon}^{n}\right)\right]^{\frac{1}{2}} \Xi\left(U_{2, \varepsilon}^{n}\right) \nabla W_{2, \varepsilon}^{n}+\left[\Xi_{M}\left(U_{1, \varepsilon}^{n-1}\right)\right]^{\frac{1}{2}} \nabla\left[\sum_{i=1}^{2} V_{i, \varepsilon}^{n-1}\right]\right|_{0}^{2} \\
& \leq \mathcal{E}\left(\left\{U_{i, \varepsilon}^{0}, V_{i, \varepsilon}^{0}\right\}_{i=1}^{2}\right)+\frac{3 \tau}{7}\left|\left[\Xi\left(U_{2, \varepsilon}^{0}\right)\right]^{\frac{1}{2}} \nabla V_{2, \varepsilon}^{0}\right|_{0}^{2}+\frac{\tau}{2 \mu}\left|\left[\Xi_{M}\left(U_{1, \varepsilon}^{0}\right)\right]^{\frac{1}{2}} \nabla\left[\sum_{i=1}^{2} V_{i, \varepsilon}^{0}\right]\right|_{0}^{2} .
\end{aligned}
$$

Therefore the desired bounds in (2.52a,b) follow immediately from (2.55) on noting (1.12), (2.19), (1.25), (2.1), (2.8), (2.12) and (2.16).

From (1.24), (2.2), (2.4a), (2.16) and (2.11) we obtain for $\eta \in W^{1, q^{\prime}}(\Omega)$ that

$$
\begin{aligned}
\mu\left(\nabla \mathcal{G}\left[\frac{U_{1, \varepsilon}^{n}-U_{1, \varepsilon}^{n-1}}{\tau}\right], \nabla \eta\right)= & \mu\left(\frac{U_{1, \varepsilon}^{n}-U_{1, \varepsilon}^{n-1}}{\tau}, \eta\right)=\mu\left(\frac{U_{1, \varepsilon}^{n}-U_{1, \varepsilon}^{n-1}}{\tau}, Q^{h} \eta\right)^{h} \\
=- & \frac{1}{3}\left(\left[\Xi_{M}\left(U_{1, \varepsilon}^{n}\right)\right]^{3} \nabla W_{1, \varepsilon}^{n}, \nabla Q^{h} \eta\right)-\frac{1}{2}\left(\left[\Xi_{M}\left(U_{1, \varepsilon}^{n}\right)\right]^{2} \Xi\left(U_{2, \varepsilon}^{n}\right) \nabla W_{2, \varepsilon}^{n}, \nabla Q^{h} \eta\right) \\
& \quad-\frac{1}{2}\left(\left[\Xi_{M}\left(U_{1, \varepsilon}^{n}\right)\right]^{\frac{3}{2}}\left[\Xi_{M}\left(U_{1, \varepsilon}^{n-1}\right)\right]^{\frac{1}{2}} \nabla\left[V_{1, \varepsilon}^{n-1}+V_{2, \varepsilon}^{n-1}\right], \nabla Q^{h} \eta\right) \\
\leq & C(M)\left[\left|\left[\Xi_{M}\left(U_{1, \varepsilon}^{n}\right)\right]^{\frac{1}{2}} \Xi\left(U_{2, \varepsilon}^{n}\right) \nabla W_{2, \varepsilon}^{n}\right|_{0}+\left|\nabla\left[\sum_{i=1}^{2} V_{i, \varepsilon}^{n-1}\right]\right|\right. \\
& \left.+\left|\left[\Xi_{M}\left(U_{1, \varepsilon}^{n}\right)\right]^{\frac{3}{2}} \nabla W_{1, \varepsilon}^{n}\right|_{0}\right]|\eta|_{1, q^{\prime}} .
\end{aligned}
$$

Similarly to (2.56), we obtain from (1.24), (2.2), (2.4e), (2.16) and (2.11) for $\eta \in W^{1, q^{\prime}}(\Omega)$ that

$$
\begin{aligned}
\mu\left(\nabla \mathcal{G}\left[\frac{V_{1, \varepsilon}^{n}-V_{1, \varepsilon}^{n-1}}{\tau}\right], \nabla \eta\right)= & -\rho_{1} \mu\left(\nabla V_{1, \varepsilon}^{n}, \nabla Q^{h} \eta\right)-\left(\Xi_{M}\left(U_{1, \varepsilon}^{n}\right) \Lambda_{\varepsilon}\left(V_{1, \varepsilon}^{n}\right) \nabla\left[V_{1, \varepsilon}^{n-1}+V_{2, \varepsilon}^{n-1}\right], \nabla Q^{h} \eta\right) \\
& -\left(\Xi_{M}\left(U_{1, \varepsilon}^{n}\right) \Xi\left(U_{2, \varepsilon}^{n}\right) \Lambda_{\varepsilon}\left(V_{1, \varepsilon}^{n}\right) \nabla W_{2, \varepsilon}^{n}, \nabla Q^{h} \eta\right)-\frac{1}{2}\left(\left[\Xi_{M}\left(U_{1, \varepsilon}^{n}\right)\right]^{2} \Lambda_{\varepsilon}\left(V_{1, \varepsilon}^{n}\right) \nabla W_{1, \varepsilon}^{n}, \nabla Q^{h} \eta\right) \\
\leq & C(M)\left[\rho_{1} \mu\left|\nabla V_{1, \varepsilon}^{n}\right|_{0}+\left|\nabla\left[\sum_{i=1}^{2} V_{i, \varepsilon}^{n-1}\right]\right|_{0}\right. \\
& \left.\quad+\left|\left[\Xi_{M}\left(U_{1, \varepsilon}^{n}\right)\right]^{\frac{1}{2}} \Xi\left(U_{2, \varepsilon}^{n}\right) \nabla W_{2, \varepsilon}^{n}\right|_{0}+\left|\left[\Xi_{M}\left(U_{1, \varepsilon}^{n}\right)\right]^{\frac{3}{2}} \nabla W_{1, \varepsilon}^{n}\right|_{0}\right]|\eta|_{1, q^{\prime}} .
\end{aligned}
$$

Using similar techniques as in $(2.56)$ and $(2.57)$ to bound $\left(\nabla \mathcal{G}\left[\frac{U_{2, \varepsilon}^{n}-U_{2, \varepsilon}^{n-1}}{\tau}\right], \nabla \eta\right)$ and $\left(\nabla \mathcal{G}\left[\frac{V_{2, \varepsilon}^{n}-V_{2, \varepsilon}^{n-1}}{\tau}\right], \nabla \eta\right)$, we obtain bounds on $\left\|\mathcal{G}\left[\frac{U_{i, \varepsilon}^{n}-U_{i, \varepsilon}^{n-1}}{\tau}\right]\right\|_{1, q}^{2}$ and $\left\|\mathcal{G}\left[\frac{V_{i, \varepsilon}^{n}-V_{i, \varepsilon}^{n-1}}{\tau}\right]\right\|_{1, q}^{2}, i=1,2$. Summing these over $n$, and noting the bounds (2.52a) yield the bounds (2.53a).

The proof of (2.53b) follows from (2.16), an inverse inequality and Sobolev embedding; see the proof of Theorem 2.2 in [1] for details. Finally the inequality (2.54) results from summing (2.46) from $n=1 \rightarrow k$, for any $k \leq N$, and noting the bounds (2.52a). 
Lemma 2.7. For $i=1,2$ let $u_{i}^{0}, v_{i}^{0} \in H_{\geq 0}^{1}$, with $u_{i}^{0} \in L^{\infty}(\Omega)$ and $u_{i}^{0}(x) \geq \zeta>0$ for a.e. $x \in \Omega$, and the assumptions (A) hold. On choosing either $\left\{U_{i, \varepsilon}^{0}, V_{i, \varepsilon}^{0}\right\} \equiv\left\{Q^{h} u_{i}^{0}, Q^{h} v_{i}^{0}\right\}$, or $\left\{U_{i, \varepsilon}^{0}, V_{i, \varepsilon}^{0}\right\} \equiv\left\{\pi^{h} u_{i}^{0}, \pi^{h} v_{i}^{0}\right\}$ if either $d=1$ or $\left\{u_{i}^{0}, v_{i}^{0}\right\} \in\left[W^{1, e}(\Omega)\right]^{2}$ with $e>2$; it follows that $\left\{U_{i, \varepsilon}^{0}, V_{i, \varepsilon}^{0}\right\} \in\left[S_{\geq 0}^{h}\right]^{2}$ with $U_{i, \varepsilon}^{0} \geq \zeta$ are such that for all $h>0$

$$
\mathcal{C}^{0}+\left(\mu G_{M}\left(U_{1, \varepsilon}^{0}\right)+G\left(U_{2, \varepsilon}^{0}\right), 1\right)^{h}+\tau \sum_{i=1}^{2}\left(\nabla \pi^{h}\left[\phi_{i}^{+}\left(U_{i, \varepsilon}^{0}\right)\right], \nabla U_{i, \varepsilon}^{0}\right) \leq C .
$$

Proof. The desired result (2.58) follows immediately from (2.52b), (2.10), (2.7), (2.11), (2.18), (1.11), (1.19), (1.18), (1.3) and (2.16).

\section{Convergence}

We set, for $i=1,2$,

$$
\begin{aligned}
& U_{i, \varepsilon}(t):=\frac{t-t_{n-1}}{\tau} U_{i, \varepsilon}^{n}+\frac{t_{n}-t}{\tau} U_{i, \varepsilon}^{n-1} \quad t \in\left[t_{n-1}, t_{n}\right] \quad n \geq 1 \\
& \text { and } \quad U_{i, \varepsilon}^{+}(t):=U_{i, \varepsilon}^{n}, \quad U_{i, \varepsilon}^{-}(t):=U_{i, \varepsilon}^{n-1} \quad t \in\left(t_{n-1}, t_{n}\right] \quad n \geq 1 \text {. }
\end{aligned}
$$

We note for future reference that

$$
U_{i, \varepsilon}-U_{i, \varepsilon}^{ \pm}=\left(t-t_{n}^{ \pm}\right) \frac{\partial U_{i, \varepsilon}}{\partial t} \quad t \in\left(t_{n-1}, t_{n}\right) \quad n \geq 1
$$

where $t_{n}^{+}:=t_{n}$ and $t_{n}^{-}:=t_{n-1}$. We introduce also $\bar{\tau}(t):=\tau$ for $t \in\left(t_{n-1}, t_{n}\right], n \geq 1$. Using the above notation, and introducing analogous notation for $W_{i, \varepsilon}$ and $V_{i, \varepsilon},\left(\mathrm{P}_{\varepsilon}^{h, \tau}\right)$ can be restated as:

Find $\left\{U_{i, \varepsilon}, V_{i, \varepsilon}\right\}_{i=1}^{2} \in\left[C\left([0, T] ; S^{h}\right)\right]^{4}$ and $\left\{W_{i, \varepsilon}^{+}\right\}_{i=1}^{2} \in\left[L^{\infty}\left(0, T ; S^{h}\right)\right]^{2}$ such that for all $\chi \in L^{2}\left(0, T ; S^{h}\right)$

$$
\begin{aligned}
& \int_{0}^{T} {\left[\mu\left(\frac{\partial U_{1, \varepsilon}}{\partial t}, \chi\right)^{h}+\frac{1}{3}\left(\left[\Xi_{M}\left(U_{1, \varepsilon}^{+}\right)\right]^{3} \nabla W_{1, \varepsilon}^{+}, \nabla \chi\right)+\frac{1}{2}\left(\left[\Xi_{M}\left(U_{1, \varepsilon}^{+}\right)\right]^{2} \Xi\left(U_{2, \varepsilon}^{+}\right) \nabla W_{2, \varepsilon}^{+}, \nabla \chi\right)\right] \mathrm{d} t } \\
& \quad=-\frac{1}{2} \int_{0}^{T}\left(\left[\Xi_{M}\left(U_{1, \varepsilon}^{+}\right)\right]^{\frac{3}{2}}\left[\Xi_{M}\left(U_{1, \varepsilon}^{-}\right)\right]^{\frac{1}{2}} \nabla\left[V_{1, \varepsilon}^{-}+V_{2, \varepsilon}^{-}\right], \nabla \chi\right) \mathrm{d} t, \\
& \int_{0}^{T}\left[\mu\left(\frac{\partial U_{2, \varepsilon}}{\partial t}, \chi\right)^{h}+\left(\left[\frac{\mu}{3}\left[\Xi\left(U_{2, \varepsilon}^{+}\right)\right]^{3}+\Xi_{M}\left(U_{1, \varepsilon}^{+}\right)\left[\Xi\left(U_{2, \varepsilon}^{+}\right)\right]^{2}\right] \nabla W_{2, \varepsilon}^{+}, \nabla \chi\right)+\frac{1}{2}\left(\left[\Xi_{M}\left(U_{1, \varepsilon}^{+}\right)\right]^{2} \Xi\left(U_{2, \varepsilon}^{+}\right) \nabla W_{1, \varepsilon}^{+}, \nabla \chi\right)\right] \mathrm{d} t \\
& \quad=-\int_{0}^{T}\left[\frac{\mu}{2}\left(\left[\Xi\left(U_{2, \varepsilon}^{+}\right)\right]^{\frac{3}{2}}\left[\Xi\left(U_{2, \varepsilon}^{-}\right)\right]^{\frac{1}{2}} \nabla V_{2, \varepsilon}^{-}, \nabla \chi\right)+\left(\left[\Xi_{M}\left(U_{1, \varepsilon}^{+}\right)\right]^{\frac{1}{2}}\left[\Xi_{M}\left(U_{1, \varepsilon}^{-}\right)\right]^{\frac{1}{2}} \Xi\left(U_{2, \varepsilon}^{+}\right) \nabla\left[V_{1, \varepsilon}^{-}+V_{2, \varepsilon}^{-}\right], \nabla \chi\right)\right] \mathrm{d} t,
\end{aligned}
$$

$$
\begin{aligned}
& \int_{0}^{T}\left[\mu\left(\frac{\partial V_{1, \varepsilon}}{\partial t}, \chi\right)^{h}+\rho_{1} \mu\left(\nabla V_{1, \varepsilon}^{+}, \nabla \chi\right)+\left(\Xi_{M}\left(U_{1, \varepsilon}^{+}\right) \Lambda_{\varepsilon}\left(V_{1, \varepsilon}^{+}\right) \nabla\left[V_{1, \varepsilon}^{+}+V_{2, \varepsilon}^{+}\right], \nabla \chi\right)\right. \\
& \left.\quad+\left(\Xi_{M}\left(U_{1, \varepsilon}^{+}\right) \Xi\left(U_{2, \varepsilon}^{+}\right) \Lambda_{\varepsilon}\left(V_{1, \varepsilon}^{+}\right) \nabla W_{2, \varepsilon}^{+}, \nabla \chi\right)\right] \mathrm{d} t=-\frac{1}{2} \int_{0}^{T}\left(\left[\Xi_{M}\left(U_{1, \varepsilon}^{+}\right)\right]^{2} \Lambda_{\varepsilon}\left(V_{1, \varepsilon}^{+}\right) \nabla W_{1, \varepsilon}^{+}, \nabla \chi\right) \mathrm{d} t \\
& \int_{0}^{T}\left[\mu\left(\frac{\partial V_{2, \varepsilon}}{\partial t}, \chi\right)^{h}+\rho_{2} \mu\left(\nabla V_{2, \varepsilon}^{+}, \nabla \chi\right)+\left(\Xi_{M}\left(U_{1, \varepsilon}^{+}\right) \Lambda_{\varepsilon}\left(V_{2, \varepsilon}^{+}\right) \nabla\left[V_{1, \varepsilon}^{+}+V_{2, \varepsilon}^{+}\right], \nabla \chi\right)\right. \\
& \quad+\left(\Xi_{M}\left(U_{1, \varepsilon}^{+}\right) \Xi\left(U_{2, \varepsilon}^{+}\right) \Lambda_{\varepsilon}\left(V_{2, \varepsilon}^{+}\right) \nabla W_{2, \varepsilon}^{+}, \nabla \chi\right)+\frac{\mu}{2}\left(\left[\Xi\left(U_{2, \varepsilon}^{+}\right)\right]^{2} \Lambda_{\varepsilon}\left(V_{2, \varepsilon}^{+}\right) \nabla W_{2, \varepsilon}^{+}, \nabla \chi\right) \\
& \left.\quad+\mu\left(\Xi\left(U_{2, \varepsilon}^{+}\right) \Lambda_{\varepsilon}\left(V_{2, \varepsilon}^{+}\right) \nabla V_{2, \varepsilon}^{+}, \nabla \chi\right)\right] \mathrm{d} t=-\frac{1}{2} \int_{0}^{T}\left(\left[\Xi_{M}\left(U_{1, \varepsilon}^{+}\right)\right]^{2} \Lambda_{\varepsilon}\left(V_{1, \varepsilon}^{+}\right) \nabla W_{1, \varepsilon}^{+}, \nabla \chi\right) \mathrm{d} t
\end{aligned}
$$


where for a.a. $t \in(0, T)$ and for all $z^{h} \in S^{h}$

$$
\begin{aligned}
& c_{1}\left(\nabla U_{1, \varepsilon}^{+}, \nabla z^{h}\right)+\left(\phi_{1}^{+}\left(U_{1, \varepsilon}^{+}\right)+\phi_{1}^{-}\left(U_{1, \varepsilon}^{-}\right), z^{h}\right)^{h}-\left(\phi_{2}^{+}\left(U_{2, \varepsilon}^{+}\right)+\phi_{2}^{-}\left(U_{2, \varepsilon}^{-}\right), z^{h}\right)^{h}=\left(W_{1, \varepsilon}^{+}-W_{2, \varepsilon}^{+}, z^{h}\right)^{h}, \quad \\
& c_{2}\left(\nabla\left[U_{1, \varepsilon}^{+}+U_{2, \varepsilon}^{n}\right], \nabla z^{h}\right)+\left(\phi_{2}^{+}\left(U_{2, \varepsilon}^{+}\right)+\phi_{2}^{-}\left(U_{2, \varepsilon}^{-}\right)+\phi_{3}^{+}\left(\sum_{i=1}^{2} U_{i, \varepsilon}^{+}\right)+\phi_{3}^{-}\left(\sum_{i=1}^{2} U_{i, \varepsilon}^{-}\right), z^{h}\right)^{h}=\left(W_{2, \varepsilon}^{+}, z^{h}\right)^{h} ;
\end{aligned}
$$

that is, $W_{1, \varepsilon}^{+}-W_{2, \varepsilon}^{+}=-c_{1} \Delta^{h} U_{1, \varepsilon}^{+}+\pi^{h}\left[\phi_{1}^{+}\left(U_{1, \varepsilon}^{+}\right)+\phi_{1}^{-}\left(U_{1, \varepsilon}^{-}\right)\right]-\pi^{h}\left[\phi_{2}^{+}\left(U_{2, \varepsilon}^{+}\right)+\phi_{2}^{-}\left(U_{2, \varepsilon}^{-}\right)\right]$and $W_{2, \varepsilon}^{+}=-c_{2} \Delta^{h}\left(U_{1, \varepsilon}^{+}+\right.$ $\left.U_{2, \varepsilon}^{+}\right)+\pi^{h}\left[\phi_{2}^{+}\left(U_{2, \varepsilon}^{+}\right)+\phi_{2}^{-}\left(U_{2, \varepsilon}^{-}\right)\right]+\pi^{h}\left[\phi_{3}^{+}\left(\sum_{i=i}^{2} U_{i, \varepsilon}^{+}\right)+\phi_{3}^{-}\left(\sum_{i=1}^{2} U_{i, \varepsilon}^{-}\right)\right]$.

Lemma 3.1. Let $\phi_{j}(\cdot)$ satisfy (1.3) with $\delta_{j}>0, j=1,2$, and the assumptions of Lemma 2.7 hold. In addition, let $\tau h^{-d\left(1-\frac{2}{p}\right)} \rightarrow 0$ and $\varepsilon h^{-d\left(\frac{1}{2}-\frac{1}{p}\right)} \rightarrow 0$ as $h \rightarrow 0$, where $p=2$ if $d=1$, and $p>2$ if $d=2$. Then there exists a subsequence of $\left\{\left\{U_{i, \varepsilon}, V_{i, \varepsilon}, W_{i, \varepsilon}^{+}\right\}_{i=1}^{2}\right\}_{h}$, where $\left\{U_{i, \varepsilon}, V_{i, \varepsilon}, W_{i, \varepsilon}^{+}\right\}_{i=1}^{2}$ solve $\left(\mathrm{P}_{\varepsilon}^{h, \tau}\right)$, and for $i=1,2$ functions

$$
\begin{array}{ll} 
& u_{i} \in L^{\infty}\left(0, T ; H_{\geq 0}^{1}(\Omega)\right) \cap H^{1}\left(0, T ;\left(W^{1, q^{\prime}}(\Omega)\right)^{\prime}\right), \\
& v_{i} \in L^{\infty}\left(0, T ; L^{2}(\Omega)\right) \cap L^{2}\left(0, T ; H_{\geq 0}^{1}(\Omega)\right) \cap H^{1}\left(0, T ;\left(W^{1, q^{\prime}}(\Omega)\right)^{\prime}\right), \\
\text { such that } & \beta^{M}\left(u_{1}\right), \beta^{1}\left(v_{i}\right) \in L^{\infty}\left(\Omega_{T}\right),
\end{array}
$$

with $u_{i}(\cdot, 0)=u_{i}^{0}(\cdot)$ in $Y_{1}, v_{i}(\cdot, 0)=v_{i}^{0}(\cdot)$ in $Y_{2}$, where $H^{1}(\Omega) \stackrel{c}{\hookrightarrow} Y_{1}, L^{2}(\Omega) \stackrel{c}{\hookrightarrow} Y_{2}$, and for a.a. $t \in(0, T)$ $f u_{i}(\cdot, t)=f u_{i}^{0}>0, f v_{i}(\cdot, t)=f v_{i}^{0}$, such that as $h \rightarrow 0$

$$
\begin{array}{lll}
U_{i, \varepsilon}, U_{i, \varepsilon}^{ \pm} \rightarrow u_{i} & \text { weak-* in } L^{\infty}\left(0, T ; H^{1}(\Omega)\right), \\
V_{i, \varepsilon}, V_{i, \varepsilon}^{ \pm} \rightarrow v_{i} & \text { weak-* in } L^{\infty}\left(0, T ; L^{2}(\Omega)\right), & \text { weakly in } L^{2}\left(0, T ; H^{1}(\Omega)\right), \\
\mathcal{G} \frac{\partial U_{i, \varepsilon}}{\partial t} \rightarrow \mathcal{G} \frac{\partial u_{i}}{\partial t} & \text { and } \mathcal{G} \frac{\partial V_{i, \varepsilon}}{\partial t} \rightarrow \mathcal{G} \frac{\partial v_{i}}{\partial t} & \text { weakly in } L^{2}\left(0, T ; W^{1, q}(\Omega)\right), \\
U_{i, \varepsilon}, U_{i, \varepsilon}^{ \pm} \rightarrow u_{i} & & \text { strongly in } L^{2}\left(0, T ; L^{s}(\Omega)\right), \\
V_{i, \varepsilon}, V_{i, \varepsilon}^{ \pm} \rightarrow v_{i} & & \text { strongly in } L^{2}\left(0, T ; L^{p}(\Omega)\right), \\
{\left[\Xi_{M}\left(U_{1, \varepsilon}^{ \pm}\right)\right]^{\alpha} \rightarrow\left[\beta^{M}\left(u_{1}\right)\right]^{\alpha} \mathcal{I},} & \text { for any } \alpha \in(0, \infty), & \text { strongly in } L^{2}\left(0, T ; L^{s}(\Omega)\right), \\
{\left[\Xi\left(U_{2, \varepsilon}^{ \pm}\right)\right]^{\alpha} \rightarrow u_{2}^{\alpha} \mathcal{I},} & \text { for any } \alpha \in(0, \infty), & \text { strongly in } L^{2}\left(0, T ; L^{s}(\Omega)\right), \\
\Lambda_{\varepsilon}\left(V_{i, \varepsilon}^{+}\right) \rightarrow \beta^{1}\left(v_{i}\right) \mathcal{I} & & \text { strongly in } L^{2}\left(0, T ; L^{p}(\Omega)\right) ;
\end{array}
$$

where $s \in[2, \infty]$ and $q=2$ if $d=1, s \in[2, \infty)$ and $q \in(1,2)$ if $d=2$.

Furthermore, if $d=1$, or $d=2$ and $\nu \geq 7$ in (1.3), then $u_{i}$ in addition to (3.4a) satisfies

$$
u_{i} \in L^{2}\left(0, T ; H^{2}(\Omega)\right)
$$

and there exists a subsequence of $\left\{\left\{U_{i, \varepsilon}, V_{i, \varepsilon}, W_{i, \varepsilon}^{+}\right\}_{i=1}^{2}\right\}_{h}$, satisfying $(3.5 \mathrm{a}-\mathrm{c}),(3.6 \mathrm{a}, \mathrm{b}),(3.7 \mathrm{a}-\mathrm{c})$ and as $h \rightarrow 0$

$$
\begin{aligned}
\Delta^{h} U_{i, \varepsilon}^{+} & \rightarrow \Delta u_{i} & & \text { weakly in } L^{2}\left(\Omega_{T}\right), \\
U_{i, \varepsilon}, U_{i, \varepsilon}^{ \pm} & \rightarrow u_{i} & & \text { weakly in } L^{2}\left(0, T ; W^{1, p}(\Omega)\right), \\
U_{i, \varepsilon}, U_{i, \varepsilon}^{ \pm} & \rightarrow u_{i} & & \text { strongly in } L^{2}\left(0, T ; C^{0, \gamma}(\bar{\Omega})\right), \quad \text { for any } \gamma \in\left(0,1-\frac{d}{p}\right),
\end{aligned}
$$

and for a.a. $t \in(0, T)$

$$
u_{i}(\cdot, t) \in C^{0, \gamma}(\bar{\Omega}) \quad \text { with } \quad u_{i}(x, t) \geq \zeta(t)>0 \quad \forall x \in \bar{\Omega} .
$$


On extracting a further subsequence, it holds also as $h \rightarrow 0$ that

$$
\begin{aligned}
& \pi^{h}\left[\phi_{j}^{ \pm}\left(U_{j, \varepsilon}^{ \pm}\right)\right](\cdot, t) \rightarrow \phi_{j}^{ \pm}\left(u_{j}(\cdot, t)\right) \quad \text { for } j=1,2, \quad \text { strongly in } C(\bar{\Omega}) \text { for a.a. } t \in(0, T), \\
& \pi^{h}\left[\phi_{3}^{ \pm}\left(\sum_{j=1}^{2} U_{j, \varepsilon}^{ \pm}\right)\right](\cdot, t) \rightarrow \phi_{3}^{ \pm}\left(\sum_{j=1}^{2} u_{j}(\cdot, t)\right) \\
& W_{i, \varepsilon}^{+}(\cdot, t) \rightarrow w_{i}(\cdot, t) \\
& {\left[\Xi_{M}\left(U_{1, \varepsilon}^{+}\right)\right]^{\frac{3}{2}} \nabla W_{1, \varepsilon}^{+} \rightarrow\left[\beta^{M}\left(u_{1}\right)\right]^{\frac{3}{2}} \nabla w_{1}} \\
& {\left[\Xi\left(U_{2, \varepsilon}^{+}\right)\right]^{\frac{3}{2}} \nabla W_{2, \varepsilon}^{+} \rightarrow u_{2}^{\frac{3}{2}} \nabla w_{2}} \\
& {\left[\Xi_{M}\left(U_{1, \varepsilon}^{+}\right)\right]^{\frac{1}{2}} \Xi\left(U_{2, \varepsilon}^{+}\right) \nabla W_{2, \varepsilon}^{+} \rightarrow\left[\beta^{M}\left(u_{1}\right)\right]^{\frac{1}{2}} u_{2} \nabla w_{2}} \\
& \text { strongly in } C(\bar{\Omega}) \text { for a.a. } t \in(0, T) \text {, } \\
& \text { weakly in } H^{1}(\Omega) \text { for a.a. } t \in(0, T) \text {, } \\
& \text { weakly in } L^{2}\left(\Omega_{T}\right) \text {, } \\
& \text { weakly in } L^{2}\left(\Omega_{T}\right) \text {, } \\
& \text { weakly in } L^{2}\left(\Omega_{T}\right) \text {; }
\end{aligned}
$$

where

$$
w_{1}-w_{2} \equiv-c_{1} \Delta u_{1}+\phi_{1}\left(u_{1}\right)-\phi_{2}\left(u_{2}\right) \quad \text { and } \quad w_{2} \equiv-c_{2} \Delta\left(u_{1}+u_{2}\right)+\phi_{2}\left(u_{2}\right)+\phi_{3}\left(u_{1}+u_{2}\right) .
$$

Proof. Noting the definitions (3.1a,b), the bounds in $(2.52 \mathrm{a}, \mathrm{b})$ and $(2.53 \mathrm{a}, \mathrm{b})$, together with $(2.58)$, imply that

$$
\begin{aligned}
\sum_{i=1}^{2}[ & \left\|U_{i, \varepsilon}^{( \pm)}\right\|_{L^{\infty}\left(0, T ; H^{1}(\Omega)\right)}^{2}+\left\|V_{i, \varepsilon}^{( \pm)}\right\|_{L^{\infty}\left(0, T ; L^{2}(\Omega)\right)}^{2}+\rho_{i}\left\|V_{i, \varepsilon}^{( \pm)}\right\|_{L^{2}\left(0, T ; H^{1}(\Omega)\right)}^{2}+\varepsilon^{-1}\left\|\pi^{h}\left[V_{i, \varepsilon}^{+}\right]_{-}\right\|_{L^{\infty}\left(0, T ; L^{2}(\Omega)\right)}^{2} \\
& \left.+\left\|\bar{\tau}^{\frac{1}{2}} \frac{\partial U_{i, \varepsilon}}{\partial t}\right\|_{L^{2}\left(0, T ; H^{1}(\Omega)\right)}^{2}+\left\|\bar{\tau}^{\frac{1}{2}} \frac{\partial V_{i, \varepsilon}}{\partial t}\right\|_{L^{2}\left(\Omega_{T}\right)}^{2}+\left\|\mathcal{G} \frac{\partial U_{i, \varepsilon}}{\partial t}\right\|_{L^{2}\left(0, T ; W^{1, q}(\Omega)\right)}^{2}+\left\|\mathcal{G} \frac{\partial V_{i, \varepsilon}}{\partial t}\right\|_{L^{2}\left(0, T ; W^{1, q}(\Omega)\right)}^{2}\right] \\
& +\left\|\left[\Xi_{M}\left(U_{1, \varepsilon}^{+}\right)\right]^{\frac{3}{2}} \nabla W_{1, \varepsilon}^{+}\right\|_{L^{2}\left(\Omega_{T}\right)}^{2}+\left\|\left[\Xi\left(U_{2, \varepsilon}^{+}\right)\right]^{\frac{3}{2}} \nabla W_{2, \varepsilon}^{+}\right\|_{L^{2}\left(\Omega_{T}\right)}^{2}+\left\|\left[\Xi_{M}\left(U_{1, \varepsilon}^{+}\right)\right]^{\frac{1}{2}} \Xi\left(U_{2, \varepsilon}^{+}\right) \nabla W_{2, \varepsilon}^{+}\right\|_{L^{2}\left(\Omega_{T}\right)}^{2} \leq C .
\end{aligned}
$$

Furthermore, we deduce from (3.2), (3.12) and (2.5) that for $i=1,2$

$$
\left\|U_{i, \varepsilon}-U_{i, \varepsilon}^{ \pm}\right\|_{L^{2}\left(0, T ; H^{1}(\Omega)\right)} \leq C \tau, \quad\left\|V_{i, \varepsilon}-V_{i, \varepsilon}^{ \pm}\right\|_{L^{2}\left(0, T ; L^{p}(\Omega)\right)} \leq C h^{-d\left(1-\frac{2}{p}\right)} \tau
$$

Hence, on noting (3.12), (3.13), $U_{i, \varepsilon}>0,(1.4)$, our assumptions on $h$ and a standard compactness result, we can choose a subsequence $\left\{U_{i, \varepsilon}, V_{i, \varepsilon}, W_{i, \varepsilon}^{+}\right\}_{i=1}^{2}$ such that the convergence results (3.4a-c), at first without the nonnegativity constraint on $v_{i}$ and the bound on $v_{i}$ in $(3.4 \mathrm{c}),(3.5 \mathrm{a}-\mathrm{c})$ and $(3.6 \mathrm{a}, \mathrm{b})$ hold. Then $(3.4 \mathrm{a}, \mathrm{b})$ and Theorem 2.4 yield, on noting (2.7), (2.11) and a standard compactness result, that the subsequence satisfies the additional initial and integral conditions.

The proof of the results $(3.7 \mathrm{~b}, \mathrm{c})$ can be found the proof of Lemma $3.1 \mathrm{in}$ [1]; and this can be easily adapted to prove (3.7a), on noting (2.17c). Furthermore, we note that Lemma 2.1 in [2] and (3.7c) imply that for $\beta^{1}\left(v_{i}\right) \geq 0$ a.e. $\Rightarrow v_{i} \geq 0$ a.e., and hence $H_{\geq 0}^{1}(\Omega)$ in $(3.4 \mathrm{~b})$, and the $v_{i}$ result in $(3.4 \mathrm{c})$.

The results (3.8)-(3.10a-f) can be proved by a simple adaption of the proof of the corresponding results in Lemma 3.1 in [3].

Theorem 3.2. Let all the assumptions of Lemma 3.1 hold. Then there exists a subsequence of $\left\{\left\{U_{i, \varepsilon}, V_{i, \varepsilon}\right.\right.$, $\left.\left.W_{i, \varepsilon}^{+}\right\}_{i=1}^{2}\right\}_{h}$, where $\left\{\left\{U_{i, \varepsilon}, V_{i, \varepsilon}, W_{i, \varepsilon}^{+}\right\}_{i=1}^{2}\right\}_{h}$ solve $\left(\mathrm{P}_{\varepsilon}^{h, \tau}\right)$, and functions $\left\{u_{i}, v_{i}, w_{i}\right\}_{i=1}^{2}$ satisfying $(3.4 \mathrm{a}-\mathrm{c}),(3.8)$ and (3.9d). In addition, as $h \rightarrow 0$ the following hold: (3.5a-c), (3.6a,b), (3.7a-c), (3.9a-c), (3.10a-c) for a.a. $t \in(0, T)$, and $(3.10 \mathrm{~d}-\mathrm{f})$. Moreover, we have that $u_{i}$ and $v_{i}$ fulfil $u_{i}(\cdot, 0)=u_{i}^{0}(\cdot)$ in $Y_{1}, v_{i}(\cdot, 0)=v_{i}^{0}(\cdot)$ in $Y_{2}$, where $H^{1}(\Omega) \stackrel{c}{\hookrightarrow} Y_{1}, L^{2}(\Omega) \stackrel{c}{\hookrightarrow} Y_{2}$. Furthermore, $\left\{u_{i}, v_{i}, w_{i}\right\}_{i=1}^{2}$ satisfy for all $\eta \in L^{2}\left(0, T ; W^{1, q^{\prime}}(\Omega)\right)$, with $q^{\prime}=2$ 
if $d=1$ and $q^{\prime} \in(2, \infty)$ if $d=2$,

$\mu \int_{0}^{T}\left\langle\frac{\partial u_{1}}{\partial t}, \eta\right\rangle_{q^{\prime}} \mathrm{d} t+\int_{\Omega_{T}}\left[\frac{1}{3}\left[\beta^{M}\left(u_{1}\right)\right]^{3} \nabla w_{1}+\frac{1}{2}\left[\beta^{M}\left(u_{1}\right)\right]^{2} u_{2} \nabla w_{2}+\frac{1}{2}\left[\beta^{M}\left(u_{1}\right)\right]^{2} \nabla\left(v_{1}+v_{2}\right)\right] \cdot \nabla \eta \mathrm{d} x \mathrm{~d} t=0$,

$\mu \int_{0}^{T}\left\langle\frac{\partial u_{1}}{\partial t}, \eta\right\rangle_{q^{\prime}} \mathrm{d} t+\int_{\Omega_{T}}\left[\left[\frac{\mu}{3} u_{2}^{3}+\beta^{M}\left(u_{1}\right) u_{2}^{2}\right] \nabla w_{2}+\frac{1}{2}\left[\beta^{M}\left(u_{1}\right)\right]^{2} u_{2} \nabla w_{1}+\beta^{M}\left(u_{1}\right) u_{2} \nabla\left(v_{1}+v_{2}\right)\right] \cdot \nabla \eta \mathrm{d} x \mathrm{~d} t$

$+\int_{\Omega_{T}}\left[\frac{1}{2} u_{2}^{2} \nabla v_{2}\right] \cdot \nabla \eta \mathrm{d} x \mathrm{~d} t=0$,

$\mu \int_{0}^{T}\left\langle\frac{\partial v_{1}}{\partial t}, \eta\right\rangle_{q^{\prime}} \mathrm{d} t+\int_{\Omega_{T}}\left[\rho_{1} \mu \nabla v_{1}+\frac{1}{2}\left[\beta^{M}\left(u_{1}\right)\right]^{2} \beta^{1}\left(v_{1}\right) \nabla w_{1}\right] \cdot \nabla \eta \mathrm{d} x \mathrm{~d} t$

$+\int_{\Omega_{T}} \beta^{M}\left(u_{1}\right) \beta^{1}\left(v_{1}\right)\left[u_{2} \nabla w_{2}+\nabla\left(v_{1}+v_{2}\right)\right] \cdot \nabla \eta \mathrm{d} x \mathrm{~d} t=0$,

$\mu \int_{0}^{T}\left\langle\frac{\partial v_{2}}{\partial t}, \eta\right\rangle_{q^{\prime}} \mathrm{d} t+\int_{\Omega_{T}}\left[\rho_{2} \mu \nabla v_{2}+\frac{1}{2}\left[\beta^{M}\left(u_{1}\right)\right]^{2} \beta^{1}\left(v_{2}\right) \nabla w_{1}+\beta^{M}\left(u_{1}\right) \beta^{1}\left(v_{2}\right)\left(u_{2} \nabla w_{2}+\nabla\left[v_{1}+v_{2}\right]\right)\right] \cdot \nabla \eta \mathrm{d} x \mathrm{~d} t$ $+\int_{\Omega_{T}}\left[\frac{1}{2} u_{2}^{2} \beta^{1}\left(v_{2}\right) \nabla w_{2}+u_{2} \beta^{1}\left(v_{2}\right) \nabla v_{2}\right] \cdot \nabla \eta \mathrm{d} x \mathrm{~d} t=0$

where for all $\xi \in H^{1}(\Omega)$ and for a.a. $t \in(0, T)$

$$
\begin{array}{r}
\int_{\Omega}\left\{\left[w_{1}(\cdot, t)-w_{2}(\cdot, t)\right] \xi-c_{1} \nabla u_{1}(\cdot, t) \cdot \nabla \xi-\left[\phi_{1}\left(u_{1}(\cdot, t)\right)+\phi_{2}\left(u_{2}(\cdot, t)\right)\right] \xi\right\} \mathrm{d} x=0, \\
\int_{\Omega}\left\{w_{2}(\cdot, t) \xi-c_{2} \nabla\left(u_{1}(\cdot, t)+u_{2}(\cdot, t)\right) \cdot \nabla \xi+\left[\phi_{2}\left(u_{2}(\cdot, t)\right)+\phi_{3}\left(u_{2}(\cdot, t)+u_{3}(\cdot, t)\right)\right] \xi\right\} \mathrm{d} x=0 .
\end{array}
$$

Proof. On choosing $z^{h} \equiv \pi^{h} \tilde{\xi}$, where $\tilde{\xi} \in W^{1, q^{\prime}}(\Omega)$, in (3.3e,f); it follows from (2.1), (2.8), (2.6), (3.5a) and $(3.10 \mathrm{a}-\mathrm{c})$ that $(3.14 \mathrm{e}, \mathrm{f})$ holds for $\xi \equiv \tilde{\xi}$. The desired result $(3.14 \mathrm{e}, \mathrm{f})$ then holds for any $\xi \in H^{1}(\Omega)$ via a density argument.

For any $\eta \in L^{2}\left(0, T ; W^{1, q^{\prime}}(\Omega)\right)$ and $\widetilde{\eta} \in H^{1}\left(0, T ; W^{1, \infty}(\Omega)\right)$, we choose $\chi \equiv \pi^{h} \eta$ in (3.3a)-(3.3d) and then analyse the subsequent terms. The desired results $(3.14 \mathrm{a}-\mathrm{d})$ follow from $(3.5 \mathrm{a}-\mathrm{c})-(3.7 \mathrm{a}-\mathrm{c}),(3.10 \mathrm{~d}-\mathrm{f}),(2.9)$ and (2.13) from a simple adaption of the proof of Theorem 3.1 in [1].

Remark 3.3. As noted in Remark 2.1 above, in the case $d=1$ one can replace $\Xi_{M}(\cdot)$ by $\Xi(\cdot)$ as the discrete entropy bound (2.54) is not required to prove (3.8)-(3.10a-f). It is then an easy matter to adjust the proofs above to show that this modified $\left(\mathrm{P}_{\varepsilon}^{h, \tau}\right)$ converges to $(3.14 \mathrm{a}-\mathrm{f})$ with $\beta^{M}\left(u_{1}\right)$ replaced by $u_{1}$.

\section{REFERENCES}

[1] J.W. Barrett and R. Nürnberg, Convergence of a finite-element approximation of surfactant spreading on a thin film in the presence of van der Waals forces. IMA J. Numer. Anal. 24 (2004) 323-363.

[2] J.W. Barrett, H. Garcke and R. Nürnberg, Finite element approximation of surfactant spreading on a thin film. SIAM J. Numer. Anal. 41 (2003) 1427-1464.

[3] J.W. Barrett, R. Nürnberg and M.R.E. Warner, Finite element approximation of soluble surfactant spreading on a thin film. SIAM J. Numer. Anal. 44 (2006) 1218-1247.

[4] K.D. Danov, V.N. Paunov, S.D. Stoyanov, N. Alleborn, H. Raszillier and F. Durst, Stability of evaporating two-layered liquid film in the presence of surfactant - ii Linear analysis. Chem. Eng. Sci. 53 (1998) 2823-2837.

[5] H. Garcke and S. Wieland, Surfactant spreading on thin viscous films: nonnegative solutions of a coupled degenerate system. SIAM J. Math. Anal. 37 (2006) 2025-2048.

[6] G. Grün, On the convergence of entropy consistent schemes for lubrication type equations in multiple space dimensions. Math. Comp. 72 (2003) 1251-1279. 
[7] G. Grün and M. Rumpf, Nonnegativity preserving numerical schemes for the thin film equation. Numer. Math. 87 (2000) $113-152$.

[8] M. Renardy, A singularly perturbed problem related to surfactant spreading on thin films. Nonlinear Anal. 27 (1996) $287-296$.

[9] M. Renardy and R.C. Rogers, An Introduction to Partial Differential Equations. Springer-Verlag, New York, 1992.

[10] A. Schmidt and K.G. Siebert, ALBERT-software for scientific computations and applications. Acta Math. Univ. Comenian. (N.S.) 70 (2000) 105-122.

[11] A. Sheludko, Thin liquid films. Adv. Colloid Interface Sci. 1 (1967) 391-464.

[12] L. Zhornitskaya and A.L. Bertozzi, Positivity preserving numerical schemes for lubrication-type equations. SIAM J. Numer. Anal. 37 (2000) 523-555. 